\title{
Noninvasive detection of lesions in the carotid artery bifurcation : a comparison between direct and indirect techniques
}

Citation for published version (APA):

van Baalen, J. M. (1987). Noninvasive detection of lesions in the carotid artery bifurcation : a comparison between direct and indirect techniques. [Doctoral Thesis, Maastricht University]. Van Gorcum. https://doi.org/10.26481/dis.19870206jb

Document status and date:

Published: 01/01/1987

DOI:

10.26481/dis.19870206jb

Document Version:

Publisher's PDF, also known as Version of record

Please check the document version of this publication:

- A submitted manuscript is the version of the article upon submission and before peer-review. There can be important differences between the submitted version and the official published version of record.

People interested in the research are advised to contact the author for the final version of the publication, or visit the DOI to the publisher's website.

- The final author version and the galley proof are versions of the publication after peer review.

- The final published version features the final layout of the paper including the volume, issue and page numbers.

Link to publication

\footnotetext{
General rights rights.

- You may freely distribute the URL identifying the publication in the public portal. please follow below link for the End User Agreement:

www.umlib.nl/taverne-license

Take down policy

If you believe that this document breaches copyright please contact us at:

repository@maastrichtuniversity.nl

providing details and we will investigate your claim.
}

Copyright and moral rights for the publications made accessible in the public portal are retained by the authors and/or other copyright owners and it is a condition of accessing publications that users recognise and abide by the legal requirements associated with these

- Users may download and print one copy of any publication from the public portal for the purpose of private study or research.

- You may not further distribute the material or use it for any profit-making activity or commercial gain

If the publication is distributed under the terms of Article $25 \mathrm{fa}$ of the Dutch Copyright Act, indicated by the "Taverne" license above, 
Noninvasive detection of lesions in the carotid artery bifurcation 



\title{
NONINVASIVE DETECTION OF LESIONS IN THE CAROTID ARTERY BIFURCATION
}

\author{
a comparison between direct and indirect \\ rechniques
}

\section{PROEFSCHRIFT}

ter verkrijging van de graad van doctor in de geneeskunde aan de Rijksuniversiteit Limburg te Maastricht, op gezag van de Rector Magnificus Prof.Dr. F.I.M. Borke, volgens het besluit wan het College van Dekanen, in het openbaar te verdedigen op vrijdag 6 februari 1987, om 16.00 uur

\section{door}

JACOB MARINUS VAN BAALEN

geboren te Hooge en Lage Zwaluwe 
Promotor: Prof. Dr. R.S. Reneman

Referenten: Prof. Dr. J.M.F.A. Mol, Heerlen

Prof. Dr. S.H. Skotnicki, Nijmegen

The financial support by P.C.M. van Baalen en W.L. Gore \& Co. B.V. for the publication of this thesis is gratefully acknowledged. 
HAN N I E

$\mathrm{R}$

E $\quad$ M

N A

PETER

I

M A R C

$N$

N

ROBERT-JAN 



\section{CONTENTS}

VOORWOORD XI

INTRODUCTION 1

CHAPTER 1. CEREBRAL CIRCULATION 3

1-1 Anatomy 3

1-2 Anatomical variations 4

1-3 Physiology 4

1-4 Collateral circulation 6

1-4-1 Extracranial collateral circulation 6

1-4-2 Extracranial to intracranial collateral circulation 7

1-4-3 Intracranial collateral circulation 7

CHAPTER 2. THE ATHEROSCLEROTIC LESION 11

2-1 The lesion 11

2-2 The ulcerated lesion $\quad 12$

2-3 Atherosclerosis and cerebral ischemia 13

2-3-1 Cerebral ischemia and embolization 13

2-3-2 Cerebral ischemia and critical stenosis 13

Conclusion $\quad 15$

CHAPTER 3. BASIC CONSIDERATIONS OF ARTERIAL BLOOD FLOW 16

3-1 Fluid energy 16

3-2 Laminar blood flow 17

3-3 Deviations from laminar flow 17

3-3-1 Bifurcations 19

3-3-2 Stenoses $\quad 21$

Conclusion $\quad 21$

CHAPTER 4. METHODS TO EVALUATE CAROTID ARTERY BIFURCATION STENOSIS 22

4-1 Doppler ultrasound in the examination of arterial blood flow 22

4-1-1 The Doppler principle 22 
4-1-2 Doppler signal processing

4-1-2-1 Audible interpretation $\quad 24$

4-1-2-2 Analogue signal processing 24

4-1-2-3 Audio frequency spectral analysis 25

4-1-3 Emission of ultrasound 25

4-1-3-1 Continuous wave Doppler flowmeters $\quad 25$

4-1-3-2 Pulsed Doppler flowmeters 27

4-2 Direct noninvasive methods of examination 28

4-2-1 Ultrasonic imaging 28

4-2-1-1 Doppler velocity imaging technique $\quad 28$

4-2-1-2 B-mode imaging technique 30

4-2-2 Direct Doppler examination and audio spectral frequency analysis of the CW Doppler signal 31

4-2-2-1 Principle and application 31

4-2-2-2 Interpretation $\quad 32$

4-2-2-3 General remarks $\quad 35$

4-2-3 Carotid Phonoangiography 38

4-3 Indirect noninvasive methods of examination 39

4-3-1 Oculoplethysmography according to Kartchner

(OPG-K) 39

4-3-2 Ocular Pneumoplethysmography according to Gee (OPG $\times$ Gee)

4-3-2-1 Principle of the OPG-Gee

4-3-2-2 Interpretation of the OPG-Gee 41

4-3-2-3 General remarks 44

4-3-3 The periorbital Doppler examination 44

4-3-3-1 Principle of the periorbital Doppler examination 44

4-3-3-2 Interpretation of the periorbital Doppler examination 45

4-3-3-3 General remarks $\quad 45$

4-4 Contrast arteriography 46

4-4-1 Technique 46

4-4-2 Reliability of contrast arteriography 47

4-4-3 General remarks 47

CHAPTER 5. METHODS OF EXAMINATION, used in the present study

5-1 Contrast arteriography

5-1-1 Technique of contrast arteriography

5-1-2 Interpretation of the arteriograms

5-2 The noninvasive examination 51

5-2-1 Ocular pneumoplethysmography $\quad 52$

5-2-1-1 Method of examination $\quad 52$

5-2-1-2 Criteria for the interpretation of the OPG-Gee 52 
5-2-2 The Doppler examinations

5-2-2-1 The Doppler instrumentation 53

5-2-2-2 The periorbital Doppler examination 54

5-2-2-2-1 Method of examination 54

5-2-2-2-2 Interpretation of the periorbital Doppler examination

5-2-2-3 The direct Doppler examination $\quad 55$

$5-2-2-3-1$ Method of examination 55

5-2-2-3-2 Interpretation of the direct Doppler examination

56

5-3 Statistical analysis

CHAPTER 6. RESULTS 60

6-1 Description of the patients 60

6-2 Results of arteriography 61

6-2-1 Arteriographic lesions in the proximal part of the brachiocephalic arteries 61

6-2-2 Arteriographic lesions at the origin of the external carotid artery $\quad 62$

6-2-3 Arteriographic lesions at the origin of the internal carotid artery

6-2-4 Arceriographic lesions in the intracranial part of the internal carotid artery 63

6-2-5 Description of the "arterial segments" 64

6-3 Results of ocular pneumoplethysmography 65

6-3-1 Comments to the results of ocular pneumoplethysmography 67

6-4 Results of the periorbital Doppler examination 70

6-4-1 Comments to the results of the periorbital Doppler examination 71

6-5 Comparison of the results of the indirect tests 72

6-6 Results of the direct Doppler examination of the internal carotid artery

6-6-1 Comments to the results of the direct Doppler examination of the internal carotid artery

6.7 Results of the direct Doppler examination of the external carotid artery and the common carotid artery

6-8 Discussion on the results of the noninvasive tests, regarding stenotic lesions in the internal carotid artery

CHAPTER 7. GENERAL DISCUSSION 
$\begin{array}{ll}\text { REFERENCES } & 104\end{array}$

CURRICULUM VITAE 126 


\section{VOORWOORD}

De basis voor het onderzoek, zoals dar beschreven is in dit proefschrift, werd gelegd in het Catharina Ziekenhuis te Eindhoven. In deze stimulerende omgeving werd de prikkel gegeven om binnen het raam van de opleiding tot chirurg actief bezig te zijn met onderzoek. Ik ben dan ook veel dank verschuldigd aan de chirurgen dr. J. Buth, J.M.M.P.H. Herman, dr. J.J. Jakimowicz en dr. J.G. Prins voor hum voortdurend aanwezige crearieve steun en interesse. In het bijzonder echter wil ik dr. Q.A.M. Eijsbouts en dr. J.G. Prins danken, die als opleiders en later dr. Q.A.M. Eijsbouts ook als directeur van het Catharina Ziekenhuis, in staat bleken de faciliteiten te scheppen die voor de ontwikkeling van de nietinvasiewe diagnostiek van het vaatstelsel essenticel zijn. Met name dr. I.J. Jakimowicz bleek vanaf het eerste begin een goede steun. Hij was rechtstreeks betrokken bij het onderzoek en dagelliks op het vaatlaboratorium aanwezig.

Zonder de gewaardeerde medewerking van de afdelingen Neurologie, Radiologie en Cardio-chirurgie zou dit werk niet tot stand zijn gekomen. De medewerking van de neurologen bleek waardevol en vruchtbaar. Ik ben speciaal dank verschuldigd aan dr. G.H.M. Landman voor de excellente wijze van samenwerking bij de vervaardiging en beoordeling van de angiogrammen en dr. J.J. Bredee voor zijn stimulerende invloed. De medewerkers van de afdeling radiodiagnostiek Ella Janssen-van der Sanden, Irene Wijnen en Harm de Vries bleken onmisbaar voor het verkrijgen van de gegevens van de afdeling radiodiagnostiek. Annette van Bergen-Boeijen bleek altijd bereid daadwerkelijke hulp te verlenen op het vataboratorium.

In het Ziekenhuis "De Goddelijke Voorzienigheid" te Sittard was de voortdurende belangstelling van dr. G.H. Hardy stimulerend. De heer P. Meuffells dank ik voor het vervaardigen van de illustraties. Ik dank de chirurgen uit Sittard voor hun begrip en geduld.

Aan de Rijksuniversiteit Limburg bleek dr. A.P.G. Hoeks een belangrijke steun bij het opzetten van de studie, en een vaste bron van informatie voor alle mogelijke aspecten van het Doppler ultrageluid-onderzoek. De medewerkers van de afdeling Medische Informatica en statistiek dr. A. Volovicz en F. Tan dank ik voor hun adviezen. Echter in het bijzonder, en zeker "last but not least" wil ik mijn promotor prof.dr. R.S. Reneman danken voor het enthousiasme vanaf het 
begin van de studie, de subtiele wijze waarop hij mijn eigenzinnigheid in banen wist te leiden, zijn helder inzicht in de problemen en zijn vermogen om dit inzicht over te dragen.

Ik dank mijn wrouw en onze kinderen voor het geduld dat zij hebben kunnen opbrengen. 


\section{INTRODUCTION}

Atherosclerotic lesions at the carotid artery bifurcation can be observed in a large mumber of patients with symptoms of cerebral ischemia. Atherosclerotic lesions of the extracranial cerebral arteries may cause symptoms of cerebral ischemia by shooting emboli originating from the lesion itself or through interference with normal blood flow, and thus the creation of a pressure drop over the stenosis. Reconstructive surgery of the extracranial carotid arteries in patients with symptoms of cerebral ischemia started in 1954. Since then operative procedures on the cervical carotid arteries have become more common in vascular surgery.

Reconstruction of the cervical carotid arteries is aimed at the relief or prevention of symptoms of cerebral ischemia. Stroke, once developed, will not be cured by surgical intervention. Therefore, it is vitally important to identify the patients with atherosclerotic lesions in their carotid arteries, at risk for cerebral ischemic events. Contrast arteriography provides an objective assessment of the presence and the severity of the carotid artery stenosis. However, this technique is not only time consuming, but also presents a risk for the patient. The development of noninvasive methods of examination was meant to circumvent the drawbacks of contrast arteriography. The ophthalmic artery, as the first larger branch of the internal carotid artery, received much attention. At first these noninvasive methods tried to detect a decrease in blood pressure distal to the stenosis in the internal carotid artery (ocular pneumoplethysmography), a delay in the arrival of the pulse waves in the branches of the ophthalmic artery or the development of collateral circulation in the periorbital arteries as a result of diminished blood pressure in the distal internal carotid artery (the periorbital Doppler examination). These examinations are performed in the periorbital region, at some distance from the lesion in the extracranial part of the cerebropetal arteries and, therefore, are called 'indirecr' noninvasive tests. More recently noninvasive tests have been developed that investigate the anatomy and the physiology of the carotid artery bifurcation itself. These "direct" tests attempt to diagnose lesions of the arterial wall by visualizing them (B-mode imaging), by detecting disturbances in the blood flow pattern as induced by the lesions (Doppler examination), or the detection of bruits (phonoangiography). In the Doppler examination high frequency ultrasound is reflected from moving red blood cells, and then changed in frequency. The Doppler ultrasound can be emitted continuously (continuous wave Doppler flowmeter) or intermittently (pulsed Doppler flowmeter). The 
Doppler signal obtained by mixing the emitted and reflected ultrasound can be processed in various ways. Especially the dawning possibilities of spectral analysis of the Doppler signal seemed promising.

The present study was performed to answer the following questions:

- can the noninvasive methods of examination accurately detect stenotic lesions in the cerebropetal arteries?

- is there a difference in the ability of the indirect and the direct tests in the detection of stenotic lesions of the origin of the internal carotid artery?

- is the noninvasive examination capable of the early detection of stenotic lesions with a minor or moderate degree of diameter reduction of the internal carotid artery?

Three noninvasive tests were selected for this study: the direct Doppler examination, with spectral frequency analysis of the Doppler signal, the periorbital Doppler examination and the ocular pneumoplethysmography (OPG-Gee). These tests were performed, after informed consent, in patients who were submitted to cerebral arteriography. The results of the three noninvasive tests were compared with the results of arteriography. As the intention of the study was not directed towards the detection of a correlation between the presence of a stenosis and the appearance of symptoms of cerebral ischemia, the neurological status of the patient was not taken into consideration. Furthermore, from the study no conclusions can be drawn about indications for reconstructive vascular surgery.

In chapter 1 the anatomy and physiology of the cerebral circulation are described. In chapter 2 some features of atherosclerotic lesions in the cerebropetal arteries and their relation to cerebral ischemia are described.

In chapter 3 the basic principles that control arterial blood flow and the disturbances of normal arterial blood flow are described, as far as they are related to the possibilities of noninvasive methods of examination.

In chapter 4 the various methods of noninvasive examination of the cerebropetal circulation are described. These methods are divided into 'indirect' and 'direct' techniques. Only" those methods used in the present study, or nowadays in common use, are described.

In chapter 5 the methods of examination, both invasive and noninvasive, as used in the present study, and the criteria used for the interpretation of the various tests are summarized.

In chapter 6 the results of the noninvasive tests are described, as compared to the results of contrast arteriography. Furthermore, the results of the various tests are discussed and compared with those obtained in other studies.

In chapter 7 the results of the noninvasive tests, their possibilities and theit limitations are discussed. Furthermore, the changing trend in the evaluation of cervical carotid artery atherosclerosis from contrast angiography towards noninvasive examination is discussed. 


\section{CEREBRAL CIRCULATION}

In this chapter some aspects of the cerebral circulation will be described to facilitate the understanding of the possibilities and the limitations of invasive and noninvasive techniques that are used for the detection of extracranial carotid artery disease.

\section{1-1 Anatomy}

Under normal circumstances the brain is supplied with blood by the internal carotid arteries and the vertebral arteries. The right common carotid artery originates together with the right subclavian artery as the innominate artery from the aortic arch. The left common carotid artery originates as a separate branch from this arch. Therefore, the left common carotid artery is slightly longer and has a short intrathoracic course. In the neck the course of the right and left carotid arteries, in their carotid sheath, is symmetrical.

The common carotid artery (CCA) divides into an external carotid artery (ECA) and an internal carotid artery (ICA) at the level of the upper border of the lamina of the thyroid cartillage, that is approximately at the level of the fourth cervical vertebra. This bifurcation is found one to three centimeters caudal to the angle of the mandibula in $63 \%$ of the cases. It may be located as low as the sixth cervical vertebra $(3,128)$. The origin of the ICA is slightly dilated as compared to its distal part. This dilation is called the carotid bulb. Usually the ICA originates from the dorsal or dorsolateral aspect of the CCA. At the right side the origin is at the medial or the dorsomedial aspect of the CCA in $18 \%$ of the cases. At the left side this situation is met in $6 \%$ of the cases (248). The ICA has no named branches in the neck. The right and the left ICA divide into the anterior cerebral artery and the middle cerebral artery. Shortly after their origin the anterior cerebral arteries are interconnected by the anterior communicating artery.

The right and the left vertebral artery arise as the first cranial branch from the right and left subclavian artery, respectively. The vertebral artery runs through the vertebral canal, which is formed by the transverse formina of the cervical vertebrae six to one. After piercing the transverse process of the atlas it forms a loop by bending laterally, backward and medially. Along its cervical course the vertebral artery gives off muscular and spinal branches. By confluence of the right and left vertebral artery the basilar artery is formed. The basilar artery divides 
into the right and left posterior cerebral arteries, after a short course at the base of the skull. The right and left posterior communicating arteries connect the posterior cerebral artery to the ICA, on the same side. This important polygonal anastomosis is called the circulus arteriosus of Willis.

Figure 1-1 shows the subtraction of a conventional arteriogram of the aortic arch.

\section{1-2 Anatomical variations}

The most frequent variations from the described pattern occur at the level of the aortic arch and in the extracranial course of the ICA.

Although the left CCA usually originates as a separate branch from the aortic arch it is in close relation to the innominate artery in $16 \%$ of the cases and may originate from this artery in $8 \%$ of the cases (38). Less frequently other variations in the origin of the brachiocephalic vessels are found. In the parients investigated in the present study in one patient the right and the left CCA had a common origin, as did the right and the lett subclavian artery, while in one patient all four arteries arose separately from the aortic arch.

According to Weibel and Fields (273) elongation of the ICA may be divided into three categories : tortuosity, coiling and kinking. In their study both coiling and kinking were present in approximately $3 \%$ of the patients. The incidence appeared to be the same in patients under and over the age of 50 years. Bilateral tortuosity was present in $15 \%$ of the patients under 50 years of age and in $25 \%$ of the patients over 50 years of age. In another large series of 1000 consecutive angiograms severe elongation of the ICA was found in $16 \%$ of the cases (196). Complete coiling of $360^{\circ}$ was found in $6.2 \%$ of the 321 patients submitted to angiography by Eikelboom and colleagues (73).

\section{1-3 Pbysiology}

The cerebral blood flow (CBF) is determined by the pressure difference between the arterial input and the venous output of the cerebrum and the cerebrovascular resistance. The pressure at the wenous side is low compared to the pressure at the arterial side. Therefore, CBF is mainly determined by mean arteriall pressure and cerebrovascular resistance. The changes in mean arterial pressure are counteracted by variations in the cerebrovascular resistance to maintain blood supply to the brain at a more or less constant level. Increases in mean arterial pressure will result in increases in the cerebrovascular resistance and vice versa. For the compensation of changes in blood pressure this regulatory mechanism is almost completely independent of structures outside the cranial cavity and is therefore called autoregulation. The autoregulatory capacity is determined by the maximal level of vasodilation and vasoconstriction of the arterioles. Mean arterial pressure changes within the range of $9.3 \mathrm{kPa}-22.7 \mathrm{kPa}(70 \mathrm{~mm} \mathrm{Hg}-170 \mathrm{~mm} \mathrm{Hg}) \mathrm{may}$ not be reflected in changes in $\mathrm{CBF}$ due to variations in the diameter of the arterioles. A decrease of mean arterial pressure below $9.3 \mathrm{kPa}$ will result in a reduction in 


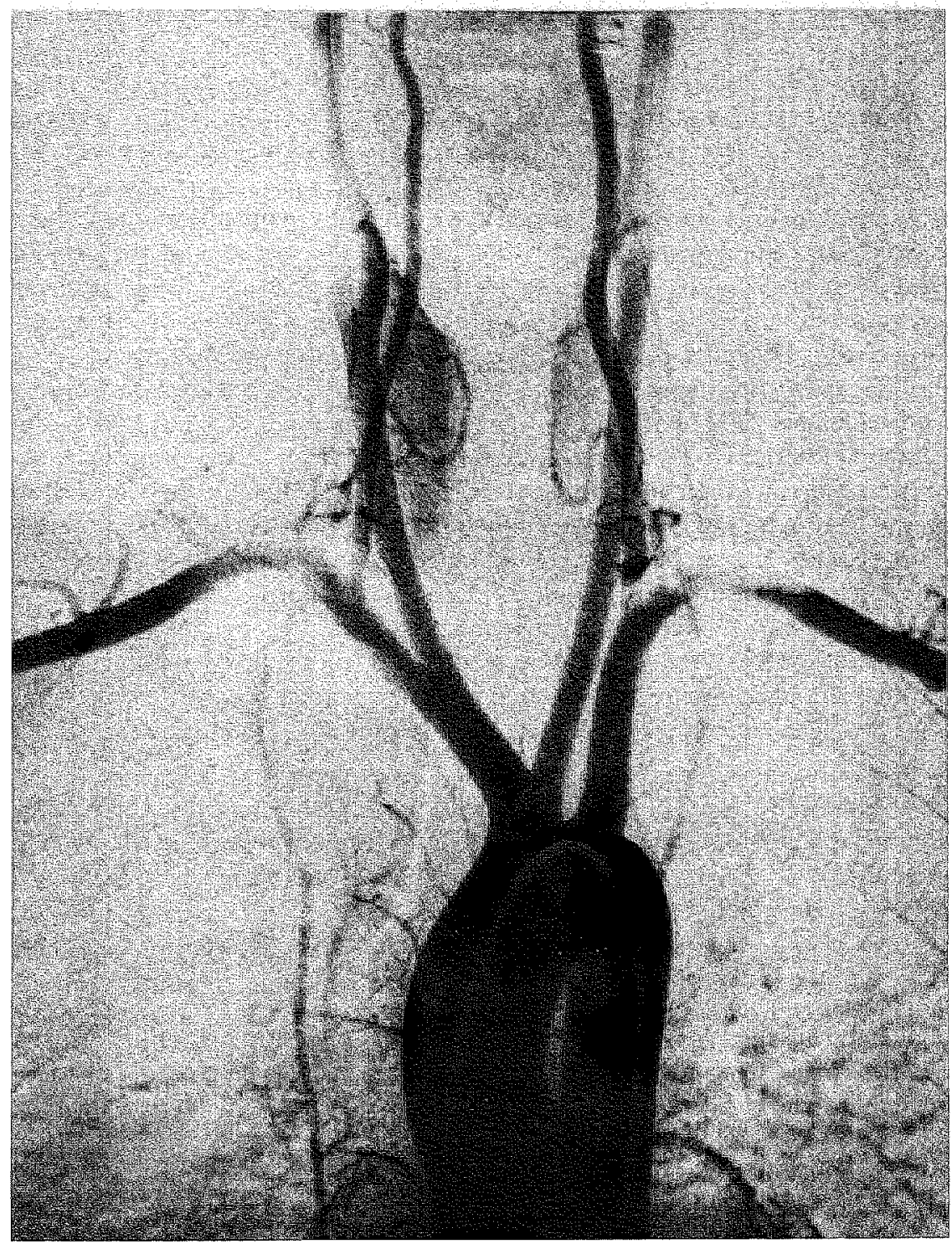

Figure 1-1. Subtraction of conventional Seldinger arteriography showing the aortic arch and the proximal part of the brachiocephalic arteries and the r branches. 
$\mathrm{CBF}$, while an increase of mean arterial pressure above $22.7 \mathrm{kPa}$ will result in a rise of $\mathrm{CBF}$ (128). Cerebrovascular resistance is also controlled by changes in arterial $\mathrm{pCO}_{2}$. Hypercapnia causes a dilation of the cerebral arterioles, while hypocapnia causes constriction. The relationship between the changes in arterial $\mathrm{pCO}_{2}$ and $\mathrm{CBF}$ is almost linear (268). A change in arterial $\mathrm{PCO}_{2}$ of $1 \%$ produces a change in total CBF of approximately $2.2 \%(128)$.

It is likely that the changes in resistance are caused by sympatheric influence on the larger cerebral vessels and by alterations in the diameter of the cerebral arterioles, mediated by neurogenic, local metabolic or mechanical (transmural pressure) stimuli to the arteriolar smooth muscle cells. Changes in CBF are not necessarily reflected in changes in the diameter of the large cervical arteries (257). By measurements, using the Kety-Schmidt method, it was shown that in man approximately $85 \%$ of the rotal CBF is supplied by the ICA's and $15 \%$ by the vertebral arteries (157). In one of the earliest reports on direct electromagnetic flow measurements on the carotid artery, Hardesty (1960) found the average flow in the ICA to be $370 \mathrm{ml} / \mathrm{min}$ in a study on 12 patients under general anesthesia. However, the measured volume flows ranged from $286 \mathrm{ml} / \mathrm{min}$ to $484 \mathrm{ml} / \mathrm{min}$ (103). Keller (142) measured volume flow at the level of the CCA. He found a volume flow of 300 to $480 \mathrm{ml}$ per minute, in healthy persons. He used a 14-channel pulsed Doppler device and calculated volume flow from the velocity profiles. Besides, he showed that abour $80 \%$ of the total volume flow occurs in the diastolic phase of the cardiac cycle and that only $20 \%$ is added by the systolic peak.

A normal value for regional CBF is $50 \mathrm{ml} / \mathrm{min} / 100 \mathrm{gm}$ (243). Important reductions in regional CBF are shown to occur with normal aging $(191,214)$, chronic atrial fibrillation (158), and increasing hematocrit values $(264,265)$ or more generally enhanced viscosity (102).

\section{1-4 Collateral circulation}

Collateral circulation may develop if obstruction occurs in one of the primary arteries. The efficacy of this collateral circulation is dependent on the development of a balance between the degree of the obstruction and the functionality of the collaterals. Therefore, the number, the severity and the localization of the obstruction(s) in the primary artery as well as the mumber and the diameter of the collaterals are important. The overall result of the collateral circulation, and thus the presence of symptoms due to hemodynamic changes caused by the obstruction, depends on the rate of development of both the obstruction and the collareral channels.

\section{1-4-1 Extracranial collateral circulation}

Extracranial collateral circulation may bypass proximal obstructions in the brachiocephalic vessels. Extensive interarterial connections exist between the 
truncus thyreo-cervicalis, the ECA and the vertebral artery. The subclavian steal phenomenon presents an example of the possibilicies of the excracranial collateral circulation in this respect. If the stenosis in the proximal part of the subclavian or innominate arteries progresses from a minimal lesion to toral occlusion several stages of blood flow disturbances in the ipsilateral vertebral artery may occur. In the vertebral artery almost normal flow with only reduced peak flow in low grade lesions (minimal narrowing of the subclavian artery) changes into reversed vertebral artery blood flow during systole (transient subclavian steal) in the presence of more severe stenotic lesions. Further progression of the degree of stenosis will result in total reversal of vertebral artery blood flow during both the systolic and the diastolic phase of the cardiac cycle (permanent subclavian steal) (218). An example of the Doppler findings in a patient with a transient subclavian steal is shown in figure 1-2.

\section{1-4-2 Extracranial to intracranial collateral circulation}

Around the orbit terminal branches of the ICA communicate with branches of the ECA. The first relatively large branch of the intracranial part of the ICA is the ophthalmic artery. It runs together with the optic nerve through the optic canal into the orbit. The branches of the ophthalmic artery are numerous. They may be divided into an orbital group and a bulbar group. Terminal branches of the orbital group are the frontal artery, the supraorbital artery and the dorsal nasal artery. These branches supply part of the forehead, the upper eyelid, and the root of the nose. They anastomose with the ECA through its terminal branches i.e. the superficial temporal artery and the facial artery. Normally the watershed between the two arterial systems lies extracranially. Therefore, the flow in the ophthalmic artery branches is directed from intracranial to extracranial. If a hemodynamically significant obstruction is present in the ICA proximal to the origin of the ophthalmic artery a shift in the watershed between the ICA and the ECA may take place. The flow in the periorbital arteries may be diminished, but, still from intracranial towards extracranial. In the presence of a high grade stenosis (substantial narrowing of the arterial lumen) in the ICA the direction of flow in the periorbital branches may reverse. The direction of flow and the flow velocity in the periorbital arteries can also be affected by stenotic lesions in the ECA. Less important connections exist between the ICA and the ECA through the anterior tympanic artery and the carotico-tympanic artery. Figure 1-3 shows the periorbital collateral pathways.

\section{1-4-3 Intracranial collateral circulation}

According to Kaplan (131) intracranial collateral circulation may exist at three levels. Firstly, through the perforating arteries to the basal nuclei. These arteries are numerous but small. Sometimes a larger commumicating branch exists between perforating branches of the anterior cerebral artery and the middle cerebral 

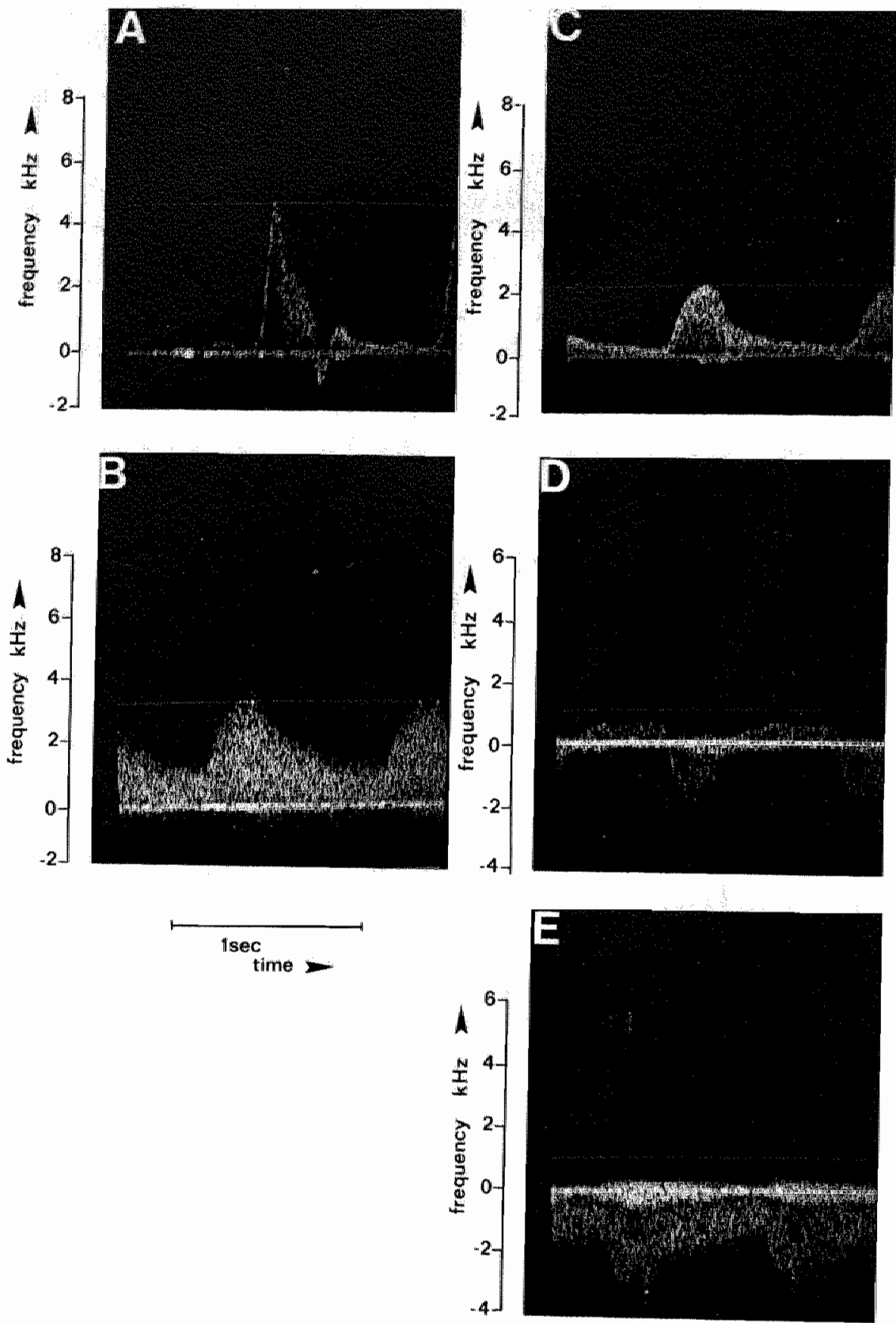
Figure 1-2. Results of the Doppler examination of the brachiall and wertebal arteries in a patient presenting a ransient subclawian steal phenomenon. The recording of the left brachal artery (A) shows a normal ri-phasic Doppler signal, and the direcrion of flow in the vertebral artery at the lef side is cephalad (B). The tecording of the righ brachial artery (C) shows the gradual upslope and the mono-phasic Doppler signal, indicative of a proximal lesion. The direction of flow in the vertebral artery at the right side (D) changes with the phases of the cardiac cycle: during diastole the direction of flow is cephalad while during systole a reversal of the direction of how is seen. During renctive hyperemia in the right arm the direction of flow in the right vertebral artery is reversed permanently (E).
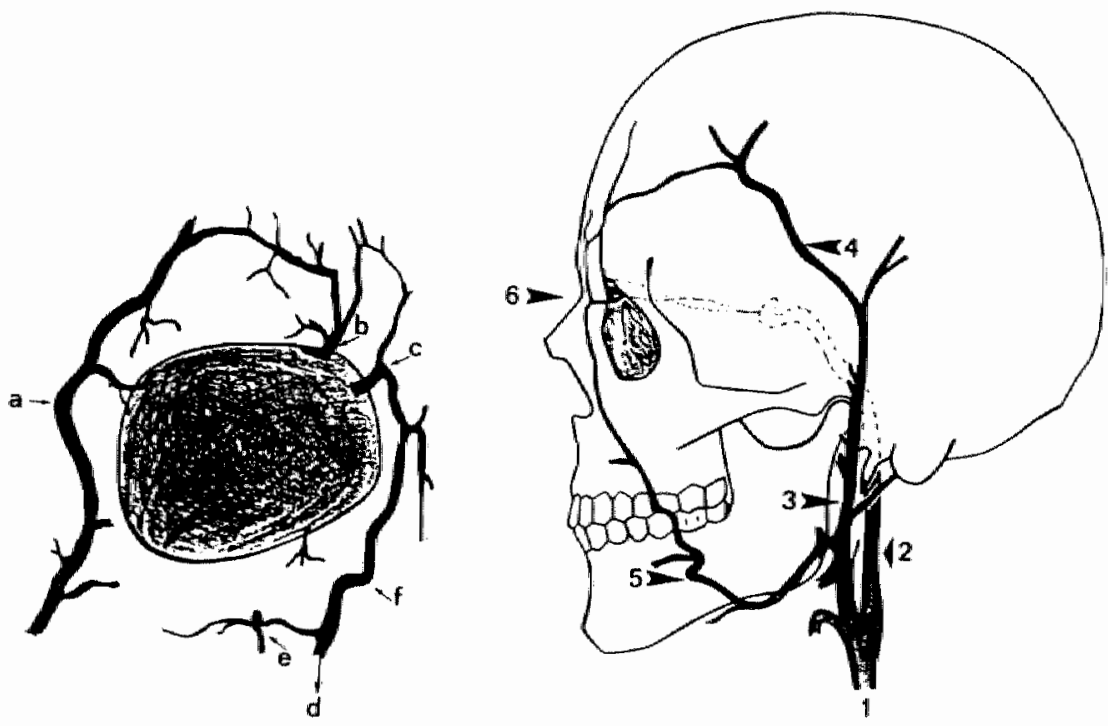

Figure 1-3. Diagram showing the anastomotic patten between teminal branches of the external carotid artery and periorbital branches of the internal carotid artery. In the nomal situation the direction of flow in the periorbital arteries is directed from within the orbita outward. On the right side a schematic diagram is shown of the warious arteries contributing to the periorbital nerwork; on the left side the branches of the extemal carotid artery in the periorbital region are shown in a frontal view of the right orbita: $\downarrow=$ common carotid artery; $2=$ internal carotid artery; $3=$ external carotid artery; $4=$ superficial temporal artery; $5=$ faciall artery; $6=$ indicates the periorbital region; a $=$ superficial temporal artery; $b=$ supraorbital antery; $c=$ frontal artery; $d=$ facial artery; $e=$ infraorbital artery; $f=$ angular artery. 
artery. Connections of these perforating branches across the midline are not found. This pathway is inadequate for extensive collateral circulation (25). Secondly, through the leptomeningeal vessells at the surface of the cerebrum. Many connections exist between the anterior, middle and posterior cerebral arteries at the borders of their supplying areas. The anastomoses are highly variable in number and size. Therefore, the efficacy of this pathway is unpredictable (69). Thirdly, through the circulus arteriosus of Willis. This polygonal anastomosis at the base of the skull has been called after Willis since he described it in 1664. It was believed to act as a kind of reserwoir for equalizing blood flow to different parts of the brain. After the introduction of invasive methods of investigation by Kramer (153) in 1912, using dyc injection, attention was drawn to the fact that each of the feeding arteries had its own perfusion area. In the case of obstruction of one of the cerebropetal arteries its supplying area could be perfused by the other arteries. It was not until 1947 that the concept of collateral circulation was acknowledged $(182,237)$.

Anatomically the circulus arteriosus is complete in about $50 \%$ of the dissected specimens $(2,213)$. In the dissections made of the circles obtained from adults with 'clinically neurological dysfunction' a complete circle was found in only $21 \%$ of the cases (234). Most frequently the variarions were found in the posterior part of the circle of Willis $(159,234)$. From radiographic investigations it is apparent that each of the anterior feeding arteries (ICA's) has its own supplying area, while both vertebral arteries perfuse the same vascular bed. The anterior and the posterior part ofthe circle are usually functionally separated from each other (156). The potency of collateral circulation was clearly demonstrated by direct electromagnetic $(122,236)$ and Doppler ultrasound $(262,280,281)$ flow measurements.

Radiographic confirmation of these collateral pathways, both extracranial and intracranial, was provided by Weibel and Fields (272) and Hawkins (108). Whether the introduction of digital subtraction angiography contributes to a better understanding of the collateral circulation remains to be seen because of its limited resolution, at least at the present state of the art, if used with intravenous contrast injections $(7,167)$. 


\section{THE ATHEROSCLEROTIC LESION}

In this chapter the relevant aspects of the atherosclerotic lesion, as far as cerebral ischemia is concerned, will be ourlined.

\section{2-1 The lesion}

Atherosclerosis is a degenerative lesion of whe arterial wall. It has been suggested that it affects primarely the incimal layer of the artery, though secondary changes in the media may occur. The atherosclerotic lesion is characterized by proliferation of smooth muscle cells, deposition of intracellular and extracellular lipids and the accumulation of extracellular collagen, elastic fibers and proteoglycans (123). In the development of a typical atherosclerotic lesion the ewolution is usually seen from fatty streaks to a firm fibrous plaque. From this fibrous plaque a complicated lesion may result by central necrosis and hemorrhage in the lesion and subsequent ulceration and formation of calcarous deposits (160). This stepwise progression, however, is not a general finding because not every fatty streak progresses to an ulcerated plaque.

Atherosclerosis is the most common cause of obstruction in the cerebropetal arteries. In their review on the cerebral arteriograms of 4748 patients with symptoms of cerebral ischemia, Hass and collaborators found atherosclerotic lesions in $80.6 \%$ of the patients (106). In $74.5 \%$ of the patients at least one of the lesions was present in the extracranial part of the cerebropetal vessels. In $40 \%$ of the cases the origin of the ICA was involved. A similar incicence and distribution was found by Blaisdell (32) in a series of 300 atteriograms, and by Hewitt (113) in a series of 174 patients with cerebral arteriography. At angiography atherosclerotic lesions of the carotid artery bifurcation were seen more often in patients suffering from cerebral ischemia than in patients with cerebral tumor (104). During autopsy studies on the carotid arteries of 961 consecutive patients fibrous changes of the intima were present in nearly $100 \%$ of the patients between 65 and 69 years of age (250). Complicated lesions, namely those containing hemorthage, necrosis, ulceration or thrombosis were found in $40 \%$ of the men and in $20 \%$ of the women of that age. 
The noninwasive examination of asymptomatic patients confirms the resulls of the morphological studies. A high incidence of flow disturbances due to stenotic lesions is found in the extracranial cerebral arteries in parients with symptoms of peripheral wascular disease (109) and in older persons (193). Stenotic lesions in the cervical carotid arteries in a series of 100 asymptomatic volunteets, varying in age from 50 to 69 years, were found in $23 \%$ of the cases by Van Merode er al (194).

\section{2-2 The ulcerated lesion}

From a clinical point of view it is important to distinguish ulcerated from nonulcerated lesions, as ulcerated lesions are inclined to cause embolization. The incidence of ulceration of lesions depends on the methods of appraisal and the time of investigation. If more than a month has elapsed between the ischemic event and surgical exploration thrombi, indicative of ulceration, were found less frequently than during immediate exploration (105).

The angiographic diagnosis of ulceration clepends on the presence of a penetrating niche, irregularities of the arterial plaque, delayed wash-out of contrast media or a well circumscribed double density of contrast superimposed on the artery (202). The ulcers may be difficult to recognize angiographically if they are smaller than $2 \mathrm{~mm}$ in diameter. Ar inspection of the surgical specimen ulceration is diagnosed as an interruption of the intimal lining, with or without thrombi or friable material, or the presence of pits or depressions with sharply delineated margins of color differences (74). Eikelboom et al (74) compared the results of arteriography and the findings at surgical exploration of 155 carotid artery plaques. At arteriography the diagnosis of ulceration was made in $54 \%$ of the lesions and at surgical inspection in $47 \%$ of the specimens. Of the 73 times that ulceration was seen in surgical specimens, it was also diagnosed at arteriography in 53 cases. In 82 specimens no ulceration was detected at gross inspection and of these lesions contrast arteriography showed signs of ulceration in 31 cases. The diagnosis of ulceration at arteriography showed a high degree of interobserver variability. Blaisdell (33) found ulceration in $76 \%$ of the arteriograms of the carotid artery bifurcation in 50 patients. The accuracy of the radiologic diagnosis proved to be $86 \%$, compared to gross inspection of the surgical specimens. The presence of ulceration cannot be related to the degree of stenosis $(33,74,263)$. Ultrasonic B-mode imaging techniques prove to be more reliable in the detection of ulceration of carotid artery stenosis than arteriography, if compared to surgical specimen $(209,274)$.

The pathologic examination of 210 surgical specimens of carotid endarterectomy revealed macroscopic ulceration, which could be seen without magnification, in $50 \%$ of the lesions. If the specimens were examined with the use of a microscope a disruption of the endothelium was present in $33 \%$ of the plaques removed. Fiften percent of these plaques also showed gross ulceration. Therefore, in only $32 \%$ of the lesions no signs of ulceration, neither macroscopically nor microscopically, could be detected (22). Using electron microscopic techniques 
Smith (249) found signs of micro-ulceration or small ulcers in 7 out of $11(64 \%)$ atherosclerotic plaques of the carotid artery bifurcation.

\section{2-3 Atherosclerosis and cerebral ischemia}

Much of the evidence linking extracranial carotid artery atheroma to cerebral ischemia is circumstantial. The atherosclerotic lesion may produce cerebral ischemia either by emboli of small particles of degenerative debris arising from a plaque or by cerebral hypoperfusion due to a substantial pressure drop over a tight stenosis (255). It is assumed that transient attacks of focal cerebral ischemia are caused by embolli rather than a hemodynamically significant obstruction at the level of the atherosclerotic lesion (67). The symptoms of global ischemia and non-hemispheric symptoms of cerebral dysfunction that cannot be explained on the basis of an embolus may be elicited by a reduction in cerebral blood flow (204).

\section{2-3-1 Cerebral ischemia and embolization}

As part of the cerebral circulation in the distribution area of the ICA may be observed at fundoscopy, the retinal arteries have drawn much attention in the investigation of cerebral ischemia. Emboli were seen traversing the retinal arteries during an attack of amaurosis fugax by Fisher in 1959 (84). Since this finding other investigators also observed and documented retinal artery emboli $(70,119)$. The actual dislodgement of a cholesterol embolus from an atherosclerotic plaque in the extracranial carotid artery was described by Barnett in 1976 (20). Whisnant (277) showed that multiple particles, arising from a single point in the carotid artery, may all lodge themselves in a particular, peripheral arterial branch. Therefore, similar, recurrent symptoms of cerebral ischemia may be produced by emboli arising from a single atherosclerotic lesion. More recently, indications of local accumulation of radio-isotopic 123-I-fibrinogen (195) and 111-In-labeled platelets $(61,99)$ were found at the site of an atherosclerotic lesion of the carotid artery bifurcation. Both fibrinogen and platelets play an important role in the formation of thrombi. The incidence of carotid artery atheroma on arteriograms of patients presenting symptoms of transient ischemic attacks (TIAs), either hemispheric or ocular, ranges from $50 \%$ to $96 \%(76,151,164,207,219,266)$.

These findings suggest that embolization may be a cause of cerebral ischemia. Figure 2-1 shows the arteriography of a carotid bifurcation with a severe, ulcerared stenosis.

\section{3-2 Cerebral ischemia and critical stenosis}

Recurrent symptoms of cerebral ischemia may be elicited by hemodynamic factors, reducing cerebral blood flow $(21,238)$. Although it is obvious that an 


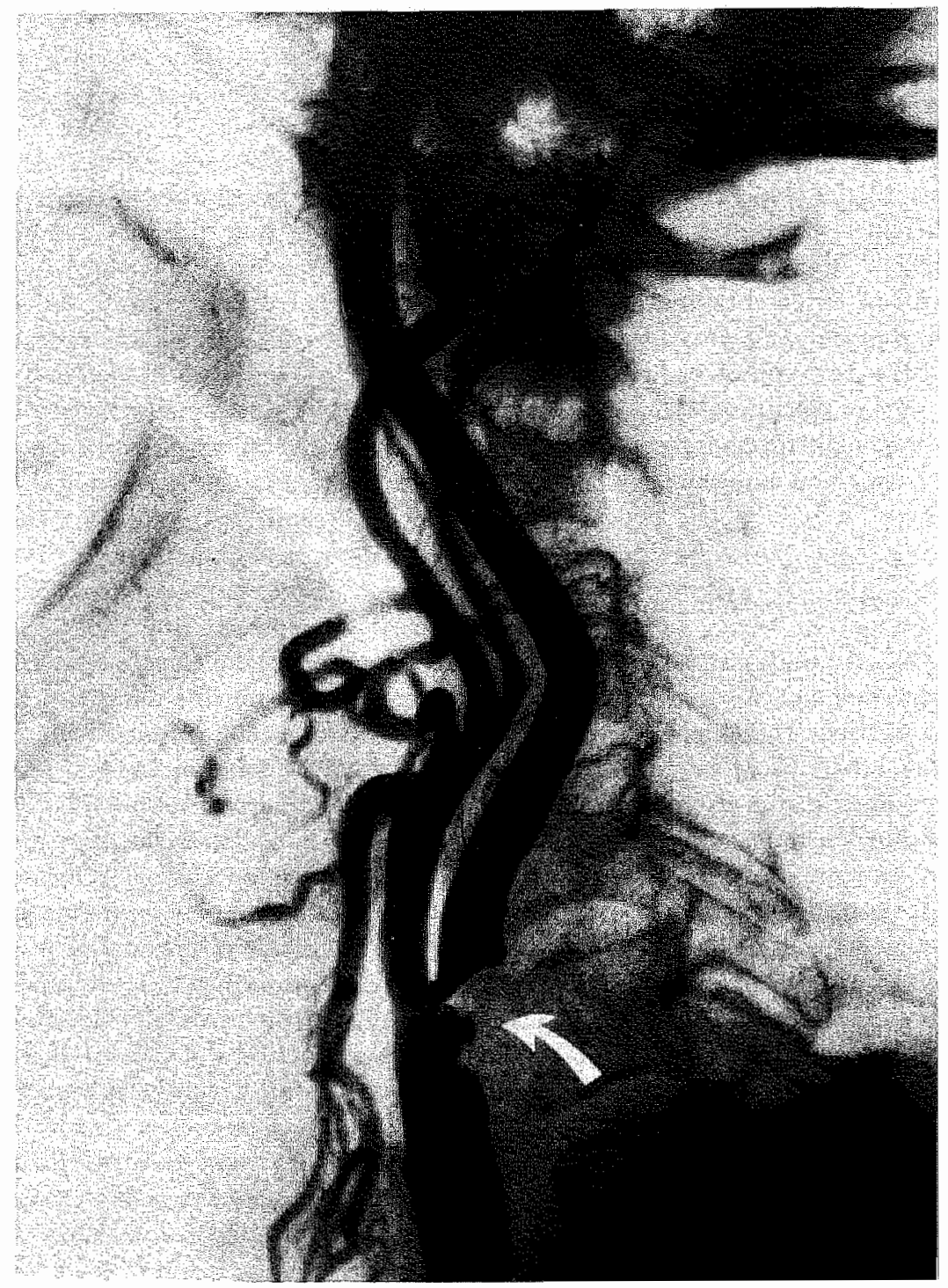

Figme 2-1. Subtraction of a conventional Seldinger arteriography of the extracranial catotid arteries showing an ulcerated lesion at the level of the carotid bifurcation. The ulcus can be recognized because of the penetrating niche in the stenotic lesion (arrow). 
atherosclerotic lesion in the cerebropetal arteries may interfere with normal blood flow the importance of a severe stenosis in generating cerebral ischemia is not unanimously acknowledged (96). Even the combination of a known tight lesion in the carotid arteries and hypotension, incluced to mimic pathophysiological disturbances in blood pressure, could not consistently generate symptoms of cerebral ischemia (144). Measurable changes in flow due to a stenosis do not occur until a certain degree of narrowing has been reached. Beyond this degree of narrowing a small additional reduction in lumen will cause a rapid reduction in flow rate and a progressive drop in pressure (26). The degree of narrowing at which flow begins to be affected has been called the 'critical stenosis'. The degree of luminal narrowing to create a critical stenosis is different. for the various vascular beds. In arteries supplying a vascular bed with a low peripheral. resistance, as in the coronary and the internal carotid systems, the critical stenosis may be reached with less severe narrowing of the lumen than in arteries with a high-resistance run-off $(26,141,145)$. The pressures proximal and distal to a stenosis, and thus the gradient across a stenosis, can easily be measured. In contrast, the flow rate can only be measured during surgery or in experimental studies. The pressure gradient that may develop across the stenosis will be noticed first during systole $(39,180,275)$.

Spencer (251) calculated the critical diameter of the carotid artery from theoretical relationships between blood flow velocity and volume flow. He found that below a diameter of $1 \mathrm{~mm}$ a critical phase is reached, and a small further decrease in lumen diameter will produce a pronounced decrease in volume flow. To describe the critical stenosis of the ICA Brice (42) considered the lumen remaining patent within the stenosis. He always found hemodynamic changes when the cross-sectional area fell below $2 \mathrm{~mm}^{2}$. More readily manageable in the clinical evaluation of patients with lesions in their extracranial cerebropetal arteries is the use of the percentage diameter reduction, as seen at contrast arteriography. A diameter reduction of the ICA of $63 \%$ or more invariably caused a pressure drop (62). If the diameter reduction ranged from $47 \%$ to $63 \%$ a pressure drop was not a consistent finding. These percentages diameter reduction may reflect much higher percentages reduction in cross-sectional area $(5,251)$.

\section{Conclustion}

- Atheroselerosis is a degenerative disease of the arterial wall that leads to focal changes in the intimall layer.

- Atherosclerotic lesions are a common finding at the carotid artery bifurcation in older persons.

- The appreciation of ulceration in lesions is difficult from angiographic studies.

- Both emboli and reduced cerebral blood flow may be related to cerebral ischemia. 


\section{BASIC CONSIDERATIONS OF ARTERIAL BLOOD FLOW}

In this chapter the implications of a stenosis, concerning detectable changes in flow velocity and pressure, are described. The physical laws governing fluid mechanics do not necessarily apply to arterial blood flow and the description is made bearing these restrictions in mind. An excellent and extensive overwiew of the physical laws concerning fluid mechanics may be found in a variety of books.

\section{3-1 Fluid energy}

Blood is moving from one point in the vascular system to the other because of the local differences in total fluid energy (52). The total fluid energy consists of potential energy and kinetic energy. The potential energy is the sum of the gravitational potential energy and the potential energy as a result of the intravascular pressure. The intravascular pressure is composed of the pressure produced by the contraction of the heart, the hydrostatic pressure and the static filling pressure of the resting circulation (17). Because the potential energy resulting from the hydrostatic pressure and the gravitational potential energy usually cancel each other, and the potential energy generated by the static filling pressure is relatively low, the intravascular pressure produced by the contraction of the heart is the most important source of potential energy. From now, in this chapter, we will consider the intravascular pressure giving rise to potential energy to be equivalent to potential energy. The kinetic energy is the energy of the blood as a result of its motion.

According to the Bernoulli theorem the total fluid energy content of a fluid particle, or a volume of fluid particles, will remain constant when moving along a streamline from one point to the other, provided a steady flow and no frictional losses. However, blood flow in the arterial tree is not steady, and the arteries are no straight, rigid pipes. Therefore, the Bernoulli theorem does only provide an approximation of the relationship between potential energy and kinetic energy. Because of viscous and inertial energy losses part of the total fluid energy is dissipated as heat. The viscous energy losses are caused by the frictional forces that exist between two neighbouring elements of a fluid that flow with different velocities. These forces are caused by the viscosity of the fluid and are greater for fluids with a high viscosity. As blood is a mixture of solid but deformable 
elements (the cells) and fluid (plasma) the viscous friction is fairly complex and varies with the hematocrit. However, blood behaves approximately as a Newtomian fluid for the velocity gradients that exist in arteries with a radius above $1 \mathrm{~mm}$ (107). The viscous energy losses are expressed by Poiseuille's law. It is important to realize that Poiseuille's law applies only to the idealized conditions of steady, laminar flow in a rigid, straight tube. From this law it is apparent that the energy loss is proportional to the length of the tube, the mean flow and the fluid viscosity and inversely proportinal to the fourth power of the radius of the tube. Therefore, the viscous energy losses are much more affected by the diameter of a conduit than by its length (145). The inerrial energy losses are due to changes in flow velocity and the direction of flow. These losses are proportional (with a proportionality constant) to the specific gravity of the blood and the square of the blood velocity (17). An important part of the total energy dissipation that occurs at stenoses is caused by the inertial energy losses. The energy losses occur at both the entrance of the lesion and the exit. The inertial energy losses are proportional to the square of the differences in blood flow velocity before, within and after the lesion (183). The first hemodynamic parameter to be influenced by the energy losses is the peak systolic blood pressure $(53,275)$. A graphic representation of the energy losses at a stenotic lesion is shown in ligure 3-1.

\section{3-2 Laminar blood flow}

In a straight pipe and under steady conditions the Newtonian fluids tend to behave according to Poiseuille's law and develop a parabolic velocity profile. When the fluid column enters a smaller segment of the system, either by branching from a larger artery or by passing through a stenosis, a new velocity profile has to develop. At the entrance of a long straight circular tube the velocity profile, describing the velocities of the fluid elements over the cross-sectional area of the vessel, will be almost flat (107). Only in a thin layer close to the wall the velocity of the blood will be affected by viscous forces. This thin layer is called the boundary layer. The thickness of the boundary layer depends on the viscosity and the density of the blood, and the frequency of the pulsatile changes. Downstream to the entrance the boundary layer becomes thicker, and the relative velocity in the core increases. After a certain length the thickness of the boundary layer approximately equals the radius of the tube (63). The velocity profile develops into a parabola $a_{n}$ representing a Poiseuille flow. The distance needed by a flat profile to change into a parabolic profile is called the entrance length. For steady flow in the human carotid artery the entrance length has been estimated to be 18 centimeters, and for pulsatile flow it is even longer (63). Hence, a truly parabollic profile will not be found in these arteries and we are dealing with the transition from flat to parabolic velocity profiles in the cervical carotid arteries. Evidence confirming this theoretical analysis was provided by Reneman et al using multi-gate pulsed Doppler systems $(227,231)$. In unsteady pulsatile flow acceleration and deceleration will have their maximal effect near the wall, i.e. in 


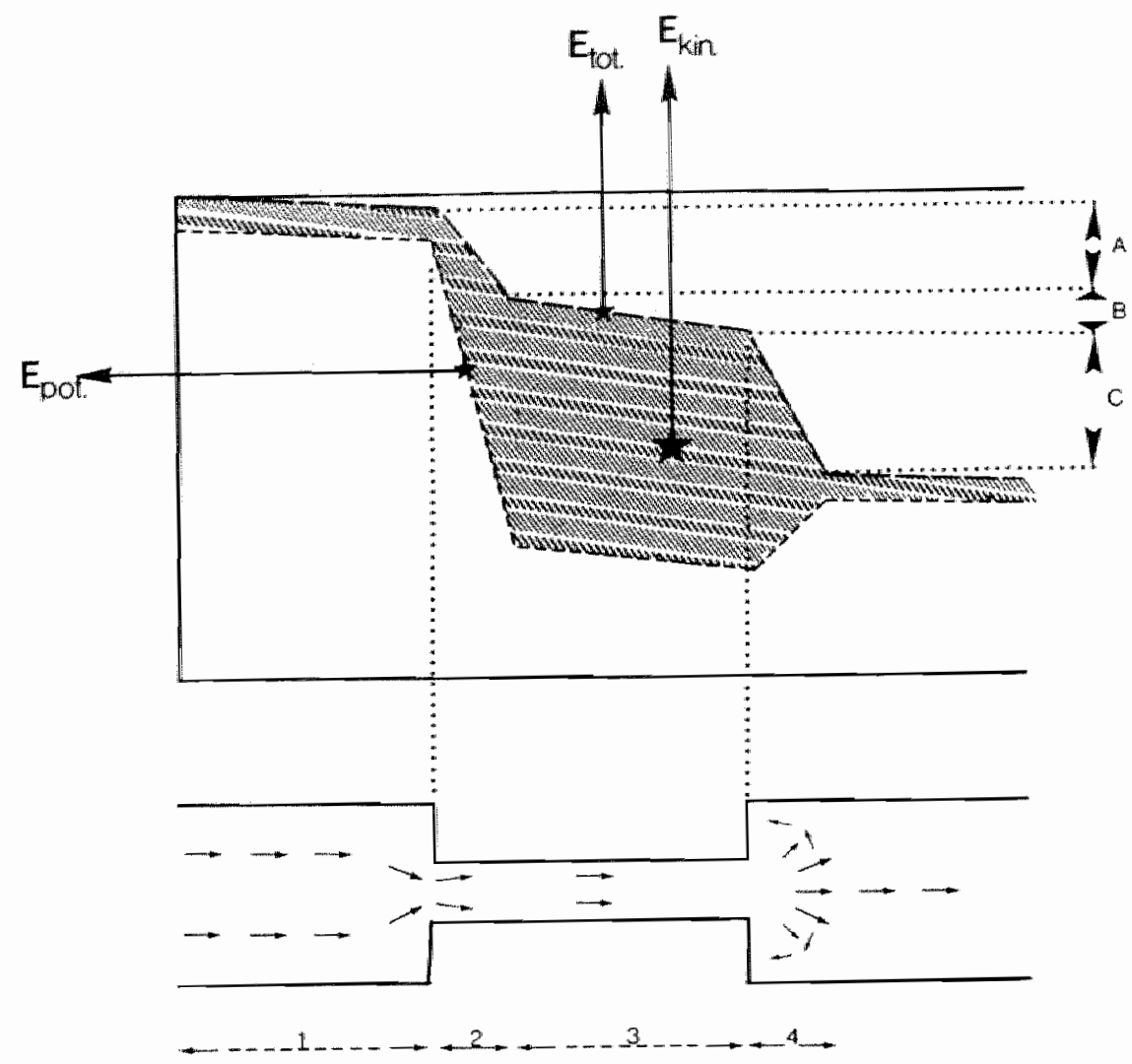

Figure 3-1. Diagram illustrating the losses of total energy $\left(E_{\mathrm{a}}\right.$ ) experienced by blood passing through a stenosis. In the prestenoric segment (1) a limited portion of energy is lost because of viscous forces. At the entrance of the stenosis (2) the blood flow velocity is increased and potential energy $\left(\mathrm{E}_{\mathrm{pos}}\right)$ is converted into kinetic energy $\left(\mathrm{E}_{\mathrm{kin}}\right)$. The loss of total energy at the entrance is represented by $A$. In passing through the stenotic segment (3) energy is lost because of friction. "The viscous energy losses in the stenosis ane represented by $B$. At the exit of the stenosis (4) energy is lost because of the conversion of kinetic energy into potential energy. The energy lost at the exit of the stenosis is represented by (C).

the boundary layer where the shear stress is highest. Acceleration adds a flat component to the velocity profile. Because of the relatively flat velocity profiles most of the red blood cells backscattering the Doppler ultrasound will have approximately the same velocity, and the Doppler signal obtained with a small sample volume will have a more or less narrow band of frequencies. During deceleration reversal of blood flow may be seen near the vascular wall. During the diastolic phase of the cardiac cycle the velocity profile is more parabolic since the 
flattening accelerative forces are missing. Therefore, a wider band of frequencias is seen in the Doppler signal. Substantial diastolic blood flow may be present in an artery if the peripheral resistance is low compared to the arterial compliance (107). Apart from the coronary arteries, diastolic flow is generally found in the $\mathbb{I C A}$, the renal artery and during reactive vasodilation.

As the flow velocity increases the laminar regularity of the $\mathbb{H}$ low may change to turbulent flow. The spontaneous transition of laminar to turbulent flow may be described by the Reynold's number. This dimensionless quantity relates the inertial forces acting on the fluid to the viscous forces. Above a Reynold's number of 2000 , measured at peak systolic velocity, local disturbances in the laminar flow pattern will result in fully developed turbulence. Below a Reynold's number of 2000 local disturbances will be damped out by the viscous forces. Since Reynold"s numbers are well below 2000 in most of the peripheral arteries turbulence is unlikely to occur under normal circumstances (260).

\section{3-3 Deviations from laminar flow}

Although disturbances in the laminar flow pattern may occur under a variety of circumstances only those related to bifurcations and stenoses will be considered. In both situations conversion of one form of energy into another, due to changes in the vascular conduit, is the basic problem. As the sum of potential energy (intravascular pressure) and kinetic energy at the different points remains constant (but for the dissipation into heat) a decrease in one form of energy will result in a consequent increase in the other form of energy, and vice wersa.

\section{3-3-1 Bifurcations}

The effect of a bifurcation is the division of the main stream into two parts. New boundary layers have to develop near and opposite to the flow divider and a new velocity profile will develop in the entrance length of the new vessels. Moreover, at bifurcations a sudden increase in the diameter of the vesscl exists. The resultant decrease in flow velocity and, therefore, kinetic energy has to be counteracted by an increase in potential energy and thus an increase in intravascular pressure (283). In addition the origin of the ICA is wider at the level of the carotid bulb, leading to a further decrease in flow velocity. This results in a small and limited region where the fluid is moving against a slight pressure gradient. As already mentioned these pressure changes will exert their maximal effect on the boundary layers, adjacent to the vascular wall. At the carotid artery bifurcation the boundary layer near the flow divider is very thin and may be neglected. The boundary layer opposite to the flow divider, however, widens because of the division of the main stream and the increase of the vessel diameter at the level of the carotid bulb. In this layer blood flow against a pressure gradient may result in a reversal of flow and thus the creation of a separation zone. Beyond this separation zone complex 


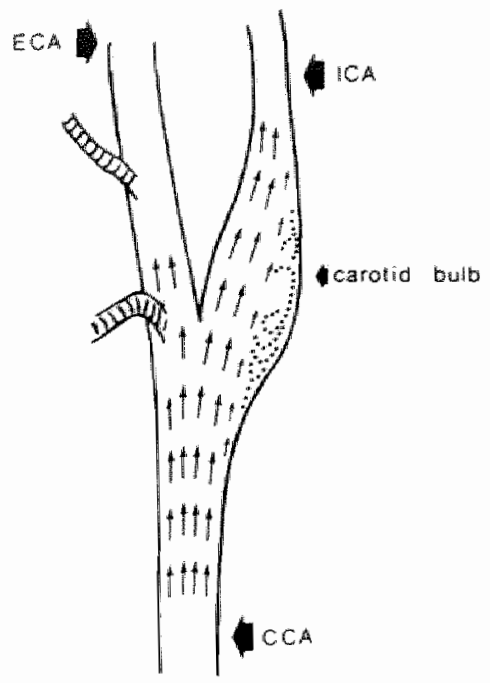

Figure 3-2. Schematic representation of the velocity patterns at the carotid artery bifurcation. The velocity profile of the common carotid artery is divided into two streams at the bifurcation. Because of the widening at the carotid bulb the formation of a new boundary layer opposite the flow divider is associated with the creation of a separation zone, resulting in an atea of recirculation wh vortices and helices. In this area the vectors of the flow velocity are not parellel with the long axis of the vessel.

flow patterns wich recirculation may develop and the laminar blood flow may become unstable $(168,175,205,284)$. If flow velocity, and thus the Reynold"s number, is high a minor wall irregularity may cause the laminar flow to become disturbed (260). A graphic impression of this recirculation at the carotid bulb is shown in figure 3-2.

As demonstrated by Ku (155) pulsatile flow creates a continuously changing region of separation, and the appearance of turbulence at the end of systole and early in diastole. The velocity patterns, as detected by Doppler ultrasound studies, in the carotid bulb of younger subjects corroborate the patterns observed in model studies $(217,231,233)$. These flow disturbances may complicate the noninvasive assessment of minor lesions at the carotid artery bifurcation $(193,231,232)$.

\section{3-3-2 Stenoses}

The effect of a stenosis is characterized by the changes in flow velocity at the entrance and distal to the stenosis associated with the conversion of one form of energy into the other (section 3-1). The kinetic energy is related to the square of 
the velocity. As the velocity of the blood at the entrance of the stenosis increases a marked conversion of potential energy into kineric energy occurs. If the stenosis has an abrupt start the streamlines will not be able to follow closely the narrowed wessel (34). Then the functional degree of stenosis, which causes the ultimate rise in velocity, will be more severe than expected from the geometry of the stenosis, due to the contraction of the streamlines. The deceleration distal to the stenosis may be augmented by the presence of a post-stenotic dilation. The increase in the diameter at this site is responsible for the fall in kineric energy and the rise in potential energy (253). This will result in a small segment where blood is flowing against a pressure gradient, as at bifurcations. The effect of the pressure gradient on the formation of new boundary layers, as the moving blood enters a new, poststenotic segment of the vessel, is identical to the situation at bifurcations. Vortices and eddies are seen in this post-stenotic area $(100,168)$. The loss of kinetic energy is not completely balanced by the increase in potential energy because of this turbulence and part of the fluid energy is dissipated into heat. Another part of the fluid energy is lost at the stenosis due to viscosity. According to Poiseuille's law the viscous energy losses within the stenotic segment are inversely related to the fourth power of the radius and 'only' directly proportional to its length. However, the application of this physical law is only permitted in certain conditions, which are not met in the human carotid artery (120), and the viscous energy losses can only be approximated by this law.

\section{Conclusion}

- blood is moving from one point to the other because of local differences in total fluid energy.

- the Bernoulli theorem relates the potential energy and the kinetic energy.

- the effect of blood moving against a pressure gradient is maximal in the boundary layers.

- the disturbances in laminar blood flow pattern, occuring at bifurcations and around stenoses, may be understood from the conversion of potential energy into kinetic energy, and vice versa. 


\section{METHODS TO EVALUATE CAROTID ARTERY BIFURCATION STENOSIS}

Although contrast angiography of the cerebropetal arteries is generally considered the golden standard for evaluation of stenotic lesions of the carotid arteries $(29,132)$ a wide variety of noninwasive methods for the detection of such lesions has been developed in recent years. These noninvasive methods may be divided into direct and indirect tests. In the direct tests the geometrical or dynamical characteristics of the carotid artery bifurcation itself are assessed. They are aiming at the detection of lesions at the carotid artery bifurcation by creating an image of the bifurcation or detecting bruits or changes in flow velocity induced by the stenosis. The indirect tests are intended to detect changes in flow velocity or pressure in the ophthalmic artery, or its branches, caused by a hemodynamically significant lesion central to the site of investigation. Hence, the indirect tests are basically unable to detect lesions of a lesser degree of stenosis, as these lesions are not reflected by changes in flow velocity, direction of flow or pressure in the periorbital arteries. Furthermore, the indirect tests are unable to localize the site of the lesion and therefore cannot differentiate between a lesion at a surgically accessable site (extracranial) or surgically inaccessable site (intracranial). Clinically, the most important difference between direct and indirect tests is the fact that direct methods can distinguish severe stenosis from total occlusion of the ICA, while indirect methods are unable to do so $(6,72,97,139,176)$.

As noteworthy results have been obtained by both direct and indirect methods of investigation some aspects of these techniques will be briefly described below.

\section{4-1 Doppler ultrasound in the examination of arterial blood flow}

Two of the three noninvasive methods of examination that were used in this study make use of the Doppler principle. The basis of this principle to measure arterial blood flow velocity will be discussed. Although only a continuous wave (CW) Doppler flowmeter was used in this study, both CW and pulsed Doppler systems will be described.

\section{4-1-1 The Doppler principle}

In Doppler flow meters a beam of ultrasonic waves is emitted from a probe. In this 
probe the ultrasound, at the $\mathrm{MHz}$ level, is generated by a piezoelectric crystal. The transmitted ultrasound is direcred through the tissue and the vessel wall into the blood stream. Part of the ultrasound is absorbed by the body tissues. The degree of absorption is different for the various tissues and is related to the frequency of the emitred ultrasound and the depth. The absorption results in an increase of the temperature of the irradiated tissue (276). Part of the emitted ultrasound is reflected from stationary and moving surfaces to either the same vibrating crystal (pulsed Doppler systems) or a second, receiving crystal (CW Doppler systems). The ultrasound reflected from starionary surfaces has the same frequency as the transmitted ultrasound. The ultrasound reflected fron moving particles, especially the red blood cells in the vessel, is shifted in frequency by an amount proportional to the velocity of the reflecting particles. This effect is known as the Doppler effect, after the Austrian scientist Christian Doppler (1803-1853). The frequency shift $\mathbb{F}_{d}$ is called the Doppler signal. The equation :

$$
\mathrm{F}_{\mathrm{d}}=\mathrm{F}_{1} \cdot \mathrm{v} \cdot \cos \mathrm{a} / \mathrm{c}
$$

relates the frequency shift to the velocity of the moving blood cells. In this equation $F_{d}=$ the frequency shift; $F_{1}=$ the frequency of the transmitted ultrasound; $\mathrm{v}=$ the velocity of the moving particles reflecting the ultrasound; $\mathrm{a}=$ the angle between the insonating sound beam and the direction of the velocity of the moving particles and $c=$ the velocity of sound in the medium.

If the particles move towards the Doppler probe the frequency of the reflected ultrasound is increased, as compared to the transmitted ultrasound. The opposite occurs when they move away from the probe. This difference can be recognized by a bindirectional Doppler flowmeter. In this technique the received signal is demodulated with two signals at the transmitter frequency shifted $90^{\circ}$ in phase with respect to each other, resulting in two Doppler signals which are $90^{\circ}$ out of phase. The sign of the phase shift between both signals indicates the sign of the frequency shift, i.e. the direction of blood flow. Although this demodulation technique provides a bi-directional velocity output, interference between forward and backward flow cannot be prevented. If the emission frequency is at the $\mathrm{MHz}$ level the Doppler signal of moving blood is in the audio range ( $\mathrm{kHz}$ level). The separation of forward and reversed flow can be achieved by further processing of the Doppler signal.The Doppler signal does not contain one single frequency but consists of a spectrum of frequencies. These variations in the frequency shift are mainly caused by the unequal velocity distribution of the red blood cells over the cross-sectional area of the vessel. As already mentioned in chapter 3 red blood cells close to the vessel wall move more slowly than those in the center of the lumen. Furthermore, variations in red blood cell interspace and the physical characteristics of the ultrasound bearm induce variations in the frequency shift (225). Both the amount of backscatter and the quantity of absorption increases with the emission frequency. This is reflected in the amplitude of the Doppler signal. Therefore, when the effects of reflection and absorption are combined a theoretical optimal frequency for the detection of arterial blood flow 
velocity at a certain depth may be found (221). Doppler flowmeters with a high emission frequency have a smaller penetration depth than Doppler flowmeters with a low emission frequency. For practical reasons, however, most Doppler flowmeters use a standard emission frequency.

\section{4-1-2 Doppler signal processing}

As discussed before the frequency shift detected with a bi-directionall Doppler flowmeter can be demodulated to present a Doppler signal in the audio range, in which the direction of flow can be recognized. This Doppler signal can be interpreted by ear, processed into an analogue velocity tracing or represented as an audio frequency spectrum.

\section{4-1-2-1 Audible interpretation}

In audible interpretation of the Doppler signal a subjective analysis is made of the characteristics of the signal. The difference in the signals obtained from the CCA, ECA and the ICA can be readily appreciated. Recognition of a stenosis can be achieved by the detection of an increase in frequency shift, particularly during the systolic phase of the cardiac cycle. Furthermore, the normally smooth sound of the ICA signal may become coarse, indicating the presence of arterial wall vibration induced by high velocity turbulence (226). The audible interpretation of carotid artery flow signals permits reliable detecrion of severe carotid artery stenosis and the distinction between severe stenosis and total occlusion of the ICA $(18,19)$. Audible interpretation of the carotid artery Doppler signals does not provide transferable criteria for the evaluation of carotid artery disease, as is needed for follow-up studies or interstudy comparison. Therefore, graphic representation of the Doppler signal is necessary.

\section{4-1-2-2 Analogue signal processing}

Determination of the mean instantaneous frequency of the Doppler spectrum and the conversion of this frequency into an electrical signal is usually performed with a zero-crossing meter. The output is an analogue voltage proportional to the number of zero-crossings per unit of time. This output can be shown as a singleline curve on oscilloscopes or physiological recorders, representing the average instantaneous velocity as a function of time. In this technique certain precautions have to be made. Firstly, a filter has to be used to separate the zero-crossings caused by blood flow from those not related to blood flow, but arising for example from random noise. Secondly, it has been shown that the zero-crossing meter consistently over-estimates the average frequency of the Doppler spectrum (215). This error depends on the shape and the width of the Doppler spectrum $(116,124,225)$. An additional disadvantage is the unreliability of the zero-crossing meter when high-amplitude, low-frequency components, as induced by vessel 
wall motion, are present in the Doppler signal. In chis situation the presence of high frequencies in the Doppler spectrum may be masked during peak systole causing flattening of the analogue signal (224) and loss of high frequency information in the Doppler signal (226). However, in spite of these limitations the analogue processing of the Doppler signal has proved to be useful in the hands of expert investigators $(50,199,218)$.

\section{4-1-2-3 Audio frequency spectral analysis}

In audio spectral analysis of the Doppler signal the various frequency shifts of the Doppler spectrum are displayed in the "sonogram". In the sonograms frequencies (on the vertical axis) are given as an instantaneous function of rime (on the horizontal axis), while the intensity of the spectral components represents the amplitude of the frequencies (as scales of grey), indicating the number of red blood cells moving at a given velocity. Therefore, the sonogram can be regarded as a three dimensional representation of the Doppler signal. Although several systems for spectral analysis of the Doppler signal have been developed $(55,59)$, the application of the Fast Fourier Transform (FFT) is commonly used nowadays. The sonogram yields information about the maximum blood flow velocity and the occurrence of various velocities of the red blood cells. Therefore, spectral analysis of the Doppler signal can be used to detect disturbances in the flow pattern $(4,29,40,226,235)$. The spread of frequencies displayed depends on the size of the sample volume in relation to the vessel diameter and the velocity profile in the artery. Because of differences in sample volume, differences in the Doppler spectra as recorded with CW and pulsed Doppler devices, have to be appreciated $(116,230)$.

\section{4-1-3 Emission of ultrasound}

In Doppler flowmeters the ultrasound can be emitted either continuously ( $\mathrm{CW}$ Doppler flowmeters) or intermittently (pulsed Doppler flowmeters). The basic differences between both systems will be described.

\section{4-1-3-1 Continuous wave Doppler flowmeters}

In CW Doppler flowmeters the ultrasound is generally transmitted from one crystal and the reflected ultrasound is received by another one (fig. 4-1). The two piezoelectric crystals (discs) are mounted adjacently in the Doppler probe. The characteristics of CW Doppler systems are determined by the geometry of the ultrasonic beams. In the transmitted beam two distinct parts can be recognized. The near field, close to the transducer, is approximately cylindrical. Beyond this near field the beam of ultrasound diverges. The length of the near field is described by the term:

$$
\mathrm{L}=\mathrm{D} \cdot 2 / 4 \mathrm{w}
$$


in which $L=$ the length of the near field; $D=$ the diameter of the transducer disc and $w=$ the wave length of the emitted ultrasound. The length of the near field can be changed by changing the diameter of the disc or by changing the emission frequency. Furthermore, by using focused transducers the working depth of the transducer can be set to be in the range of the near field (222). As the transducer is only half a disc in most CW Doppler probes the distribution of ultrasound in the beam is not axi-symmetric and the boundaries of the ultrasonic beam are not as distinct as shown in figure $4-1$ (116). Because the principle of the ultrasonic beam can be applied to both the emitting and the receiving crystal, there is a certain area in which these two beams overlap (fig. 4-1). This area can be described as the

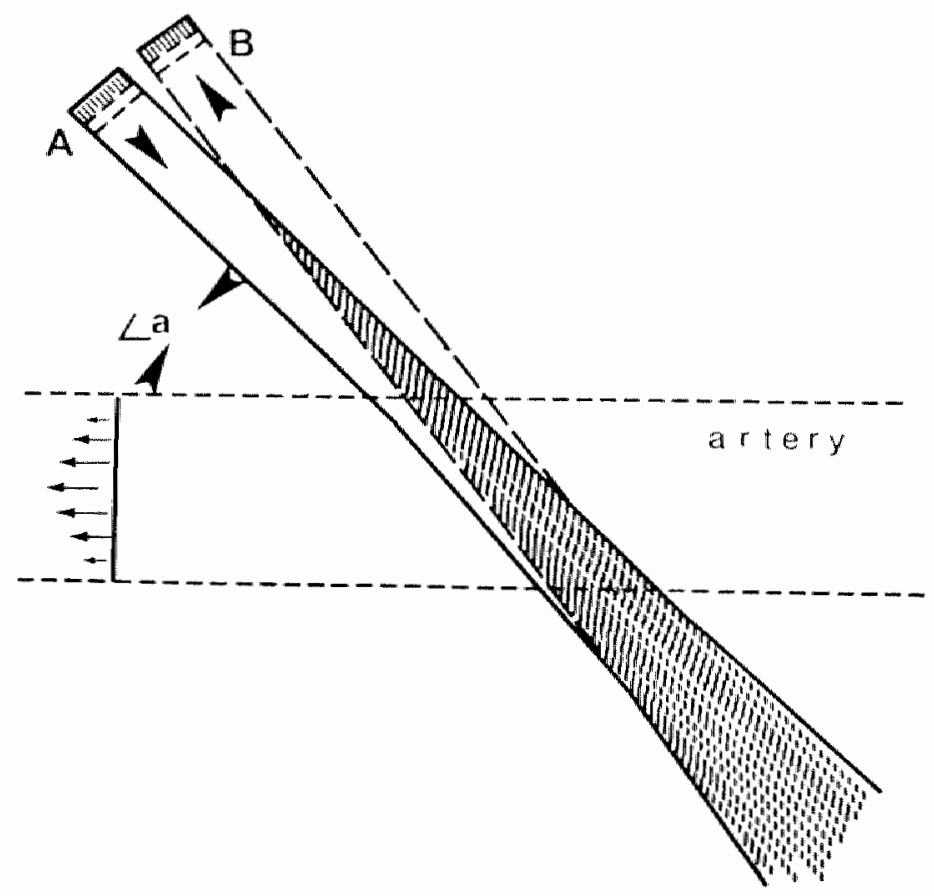

Figure 4-1.Schematic illustration of the emitting (A) and receiving (B) ultrasonic beams of the continuous-wave Doppler probe. The shaded area represents the overlap of the two beams. In this area the detection of blood flow velocity is possible. The angle between the ultrasonic beams and the veccor of the flow velocity (a) influences the frequency shift of the
Doppler signal. 
"sampling area" of a CW Doppler system, in which flow can be detected. In this sampling area part of an arterial cross-section, the entire arterial cross-section, or the cross-sections of multiple vessels can be present (fig. 4-2). Even if the information obtained in the sonogram arises from one vessel, it generally arises from both the center of the lumen and the boundary layers. In the Doppler signal this will be represented in a wider band of frequency shifts. Because the low frequency reflections, originating from vessel wall movements, may disturb the shape of the frequency spectrum a high-pass filter with a lower cut-off frequency of $300 \mathrm{~Hz}$ that can be switched "on" or "off" is necessary (228).

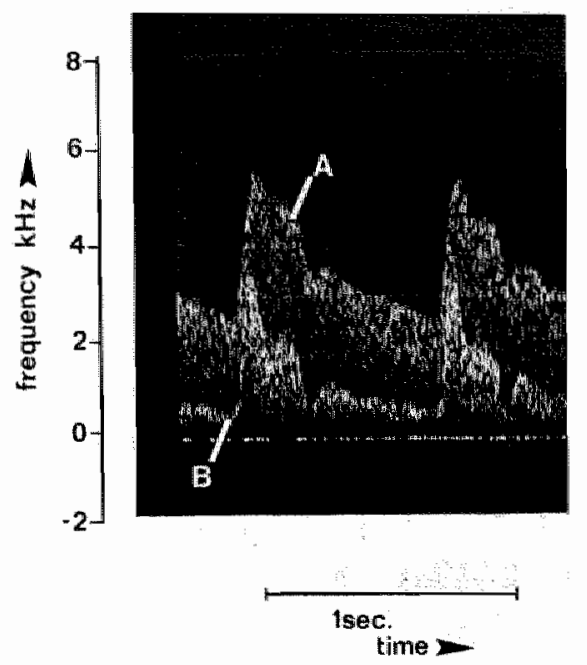

Figure 4-2. Sonogram of a continuous-wave Doppler signal containing reflections from: the internal carotid artery (A) and the excernal carotid artery (B).

In this sonogram the notch between the systolic and the diastolic phase of the cardiac cycle can be observed. The difference in the depth of this notch in the sonogram of the internal. and external carotid artery is evident.

\section{4-1-3-2 Pulsed Doppler flowmeters}

In pulsed Doppler flowmeters the ultrasound is intermittently transmitted from a piezoelectric crystal. Between the emissions of two succeeding bursts of ultrasound the same crystal is used to receive the reflected ultrasound from the previous emission. Because the same crystal is used for transmission and reception a limit is set to the maximum pulse repetition frequency. An electronic gate allows the selection of reflections of the ultrasound from a certain distance from the transducer (229). The size of the sample volume depends on the axial resolu- 
tion, which is primarely determined by the duration of emission, and the lateral resolution, which is determined by the width of the ultrasonic beam (116). Both resolutions should be in the same order to obtain the best results. In pulsed Doppler systems the size of the sample volume is small compared to the size of the insonated artery and it can be positioned in the center of the vessel. This eliminates interference of the Doppler spectrum with vessel wall movements and diminishes interference with the slowly moving red blood cells in the boundary layer. Therefore, the Doppler spectrum shows a relatively narrow band of reflected frequencies. A drawback of a small sample volume may be the low signal-to-noise ratio. An example of the differences between the Doppler spectra as obtained with a CW Doppler and a pulsed Doppler flowmeter in the same vessel in the same person is shown in figure $4-3$. The major limitation of current pulsed Doppler systems is the finite travel time of sound pulses in tissue (282). The maximum Doppler frequency shift that can be detected unambiguously depends on the transmitter frequency and the distance between transducer and vessel $(115,225)$. The distance sets an upper bound to the pulse repetition frequency, which should exceed the maximum Doppler frequency at least twice (228). Recently, Hoeks and colleagues (117) indicated new possibilities to circumvent these limitations. A more detailed description of pulsed Doppler systems is considered beyond the scope of this study, which was performed with a CW Doppler flowmeter.

\section{4-2 Direct noninvasive methods of examination}

The goal of the direct methods is to obtain either an image of the carotid artery bifurcation itself or information about physiological phenomena as may be induced by a stenotic lesion at the level of the bifurcation.

\section{4-2-1 Ultrasonic imaging}

Carotid artery imaging may be performed in two ways: velocity imaging with Doppler techniques or B-mode imaging of the vessel wall (287).

\section{4-2-1-1 Doppler velocity imaging technique}

The Doppler imaging devices produce an image of the carotid artery bifurcation by storing on an oscilloscope or in a (digital) videomemory the positions where flow velocities can be detected. To detect flow velocities a Doppler flowmeter is repeatedly moved along the carotid artery bifurcation. The position of the transducer of the Doppler flowmeter is electronically sensed by a bi-plane position sensing arm and transmitted to the storage medium. This positionsensing arm limits the movements of the transducer in two perpendicular planes. Only the positions with a flow velocity exceeding a certain threshold level are stored. To improve the quality of the image a bi-directional Doppler flowmeter 

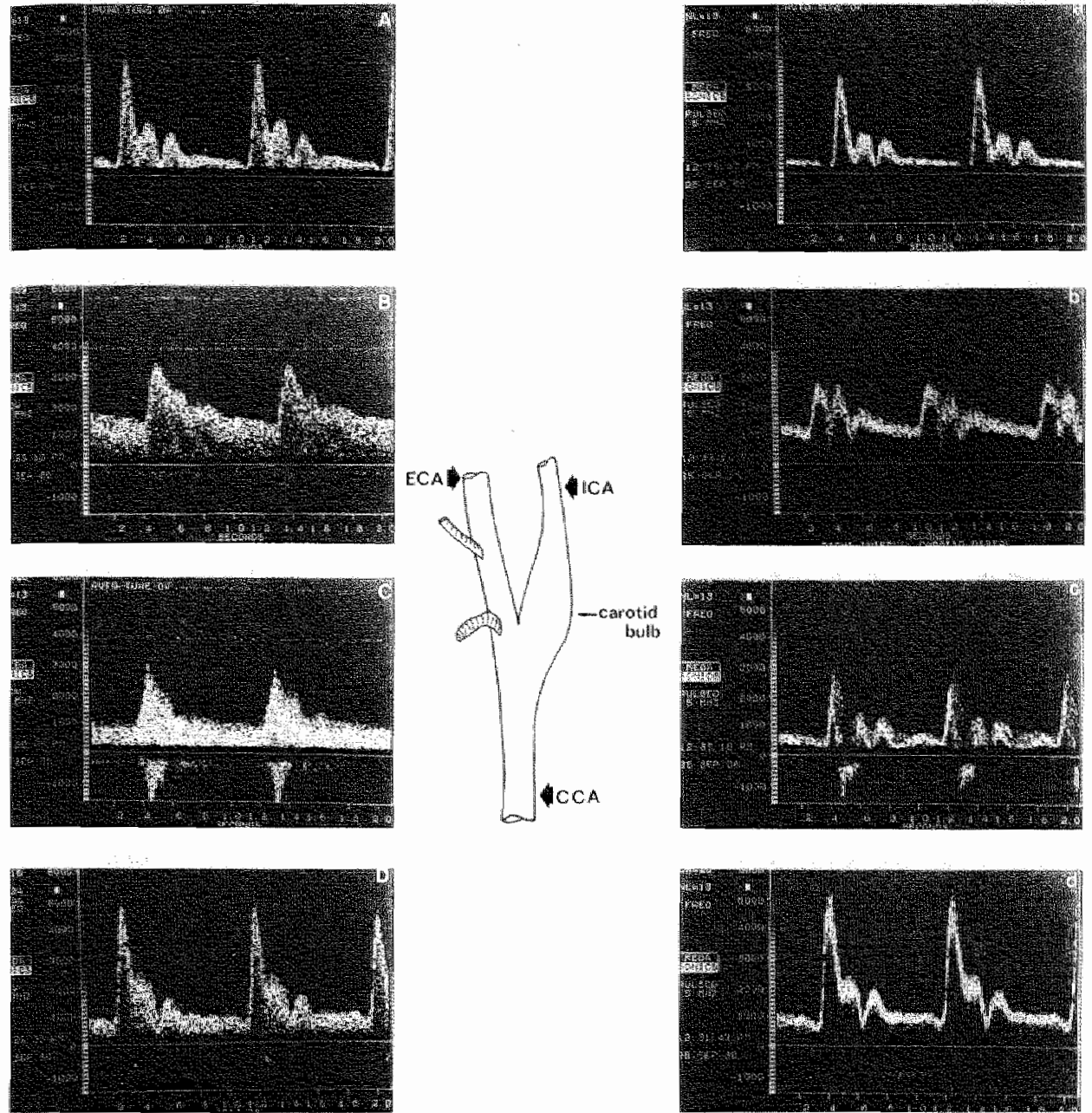

Figure 4-3. Sonograms of the continuous wave Doppler signals (on the left side) and the pulsed Doppler signals (on the right side) of the external carotid carotid artery, internal carotid artery, carotid bulb and the common carotid artery in the same person. The differences in the width of the Doppler spectrum are obvious.

should be used. Although the image obrained resembles the carotid artery bifurcation at arteriography it is based upon physiological data (blood flow velocity derected by a Doppler flowmeter) rather than anatomical structures. These Doppler imaging systems can be coupled to CW $(165,251,271)$ or pulsed $(31,114)$ Doppler flowmeters. The diagnosis of a stenotic lesion depends on the apprecia- 
tion of increased frequencies in the Doppler signal secondary to an increased flow velociry at a certain site in the image. The recognition of the increased velocities may be facilitated by using three different colours for positions with different levels of frequency shifts $(60)$. However, the use of spectral frequency anallysis of the Doppler signal may be more accurate than the use of three arbitrary threshold levels $(125,226)$. The use of Doppler velocity imaging without additional spectral frequency analysis seems less satisfying for the detection of stenoses (287). From studies in which the results of CW $(127,173,289)$ and pulsed $(15,169,259)$ Doppler imaging devices are compared to contrast arteriography it seems that there is a fair agreement between both Doppler systems in the detection of lesions of $50 \%$ stenosis or more. In the detection of lesions with a lesser degree of stenosis pulsed Doppler systems are likely to be more accurate than CW Doppler systems. The major advantage of Doppler velocity imaging is its use in localization and documentation of the site of the Doppler signal under examination.

A number of pitfalls can occur with carotid artery velocity imaging. These may be related to the local anatomy because superimposition of the ECA and the ICA in the coronal plane can create a false impression of carotid artery occlusion. Furthermore, discontinuity of vessel images may occur in normal persons because of elongation (tortuosity) of the ICA. Also errors may be introduced if the ICA is occluded and one of the branches of the ECA may be mistaken for the normal ICA (286). Another possibility of misinterpretation is introduced by strongly attenuating plaques which may cause low-amplitude, weak or absent Doppler signals along parts of the carotid artery bifurcation and lead to considerable difficulty in mapping the vessels (252). By combining the Doppler imaging technique to analysis of the Doppler signal, preferably audio spectral frequency analysis, some of these difficulties can be overcome (125).

\section{4-2-1-2 B-Mode imaging technique}

The B-mode imaging techniques are based on the concept that ultrasound traversing biologic tissue is reflected from interfaces between structures with different acoustic impedances. In carotid artery imaging ultrasound is reflected from the vascular wall. Therefore, it presents true anatomic information. In the evaluation of $\mathrm{B}$-mode images the atherosclerotic plaques are identified as echogenic material within the lumen of the vessel. The advantage of this technique is its ability to detect lesions, localize them in relation to the carotid bifurcation and indicate their size. In addition, B-mode imaging offers the potential for the detection of surface iregularities indicating the existence of ulcerations which may be held responsible for embolization. However, the presently available instrumentation may need further improvement of the resolution $(270,288)$. The limitations of this technique are related to the inability of ultrasound to differentiate unambiguously between substances of the same acoustic impedance and therefore to distinguish a soft fatty plaque or a recent thrombus from flowing blood $(56,288)$. This implies that a significant stenosis may be seriously underesti- 
mated if the lesion contains a large amount of soft fatty material and a recenty totally occluded vessel may appear as normal on the image. Large acoustical interfaces also create significant problems. When a calcified plaque is situated on the anterior wall of the vessel it prevents further penetration of the ultrasound and a large acoustical shadow is created in which it is impossible to visualize the vessel (192). It should also be noted that only surfaces of a wessel that are relatively perpendicular to the sound beam can be imaged. Therefore, it may be necessary to examine the carotid arteries from different angles of insonation. Estimation of the degree of stenosis is influenced by the plane of section, as was demonstrated by Zwiebel (288).

Many of the problems may be overcome by coupling a pulsed Doppler device to a B-mode imager (duplex scan) $(40,77,78,216,285)$. Through the combination of $\mathrm{B}$-mode imaging and pulsed Doppler systems the peak velocities in the CCA and at the site of the stenosis in the ICA can be measured with approximately the same angle of insonation. If done so the ratio of the mean peak velocities in the ICA and the CCA appears to increase linearly with the degree of stenosis (31). The accuracy of the duplex scan in the detection of carotid artery stenosis has been established $(41,79,98,149,163)$.

4-2-2 Direct Doppler examination and audio spectral frequency analysis of the CW Doppler signal

Since early reports on the use of Doppler flowmeters in the examination of arterial blood flow velocity this technique has caused revolutionary changes in the evaluation of patients with arterial disease $(85,242)$. Although in the evaluation of extracranial cerebrovascular disease a vast experience has been gained with analysis of the velocity waveform of the CCA $(198,240)$ the introduction of audio spectral frequency analysis has enabled a more direct evaluation of lesions of the carotid artery bifurcation.

\section{4-2-2-1 Principle and application}

The direct Doppler examination of the cervical carotid arteries is focused on the detection of the blood flow characteristics in these arteries. These blood flow characteristics are assessed from the sonogram. To obtain an optimal sonogram the angle of insonation is adjusted in two perpendicular planes so that the sonogram displays the maximal peak systolic frequency detectable. The examination is started over the CCA low in the neck. The Doppler probe is moved along the artery until the carotid artery bifurcation is reached. The Doppler signal obtained at the carotid artery bifurcation is lower pitched and may have a 'bubbling' sound. Even in the Doppler signal of the carotid artery bifurcation of young, healthy adults spectral broadening may occur, with the appearance of negative frequency shifts (figure 4-4). These negative frequency shifts represent the already described (section 3-3-1) vortices and helices, present in and beyond 

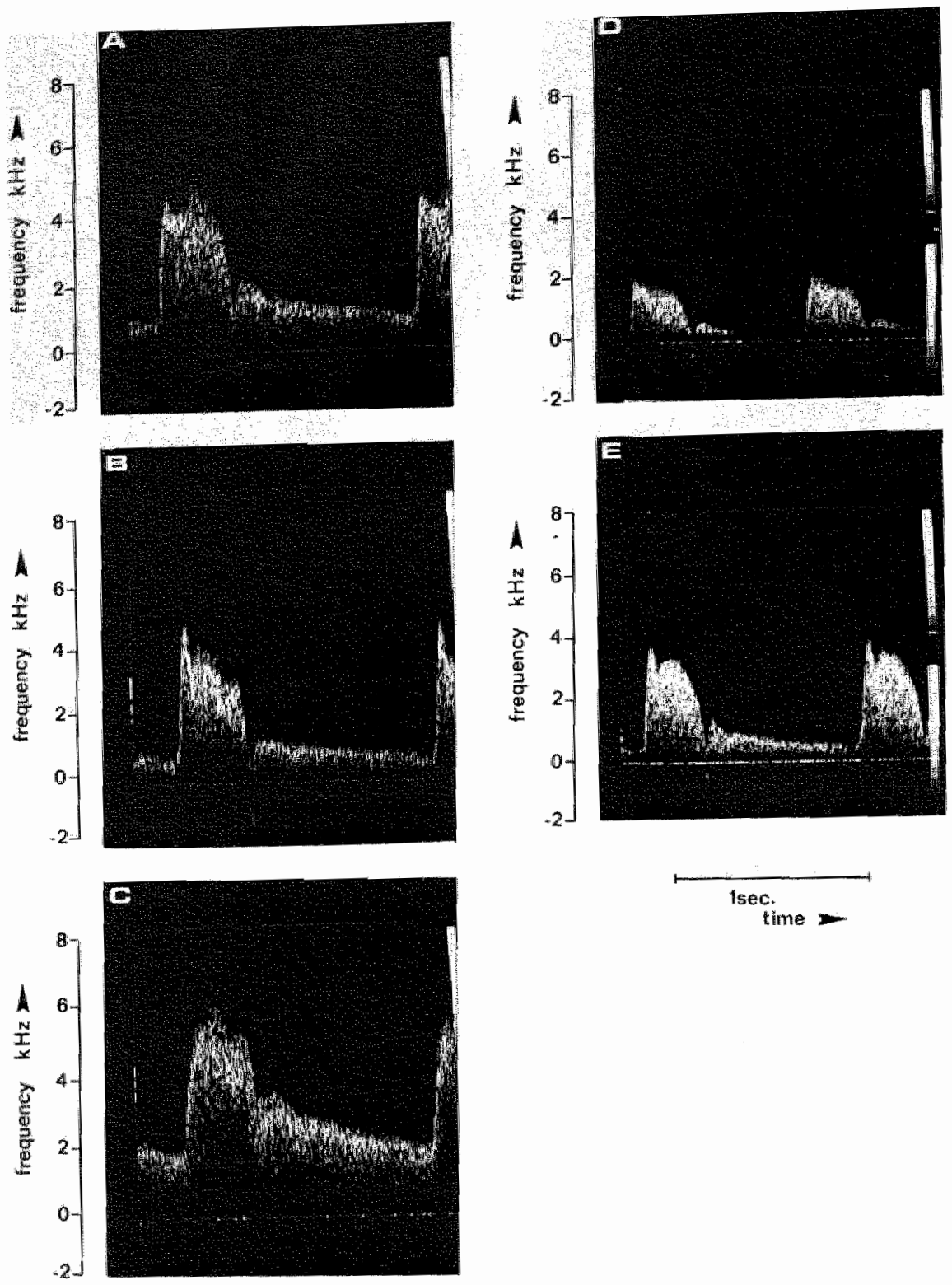

1sec.

time

Figme 4-6. Sonograns of the contrnuous-wave Doppler signals of the carotid arteries. On the left side sonograms of the common carotid artery (A), the external carotid artery (B) and the interal caroud artery (C) are shown, as may be observed in the normal situation. "The window beneath the oudine of the Doppler signal is clearly visible. In the sonogram of the common carotid artery the presence of diastolic blood flow is reflected by a positive 
investigation the Doppler signal will show disturbances as compared to the normal pattern. Then, at the site of the lesion the frequency shift will rise in proportion to the severity of the stenosis due to an increased flow velocity. This will be most obvious during systole. In addition, distal to the stenotic lesion a second phenomenon may be noticed. Because of the turbulence and eddy currents occurring distal to the lesion the reflected ultrasound will anise from red blood cells with various flow velocities. This will be reflected in the sonogram by broadening of the Doppler spectrum, and the disappearance of the systolic window (figure 4-7). In the Doppler signal a gruff sound may then be heard. Of course, in the presence of a total occlusion no Doppler signal can be obtained.

\section{4-2-2-3 General remarks}

The direct Doppler examination of the cervical carotid arteries is a safe method of investigation. However, the examination does require some skill and understanding of the principles of the Doppler examination. The examination provides both an audible and a visual input to the examiner and therefore the technique of examination can be learned in a limited period of time. The examination should be started by examining the brachial arteries to detect cardiac arythmias and more centrally located lesions (199). The possibility to recognize and identify the various cervical carotid arteries under investigation by an audible interpretation of the Doppler signal has been accepted $(18,19,125)$. According to Spencer (254) certain secondary changes in the Doppler spectrum may be observed. Firstly, the presence of an a-sonic gap without a rise in peak systolic frequency and in combination with spectral broadening, is thought to be indicative of a nonstenotic calcified plaque. If the calcified plaque is stenotic this may be concluded from the increased peak systolic Doppler frequency shifts distal to the a-sonic gap. Secondly, locally inverted Doppler signals and mirroring of Doppler frequency shifts, indicating forward and back ward flow within the same heart cycle, may be associated with non-stenotic plaquing. The inversion of the Doppler may also be noticed as a result of coiling of the artery. However, then it will often be associated with variations in the peak systolic frequency as the ultrasonic beam insonates the different parts of the elongated vessel with different angles of insonation. Figure 4-8 shows some secondary changes in the Doppler spectrum.

frequency shift at the end of diastole. In the sonogram of the common carotid artery (A) the post-systolic notch takes an intermediate pattern compared to the notch observed in the external carotid artery and the internal carotid artery. On the right side sonograms of the common carotid artery (D) and the external carotid artery (E) are shown in the case of an occlusion of the internal carotid artery. In the sonogram of the common carotid artery the frequency shifrs, indicating bllood flow velocity, occur only early in diastole. At the end of diastole no reflections are seen in the sonogram. The post-systolic notch in the latter sonogram reaches to the zero level, contrast to this notch in the sonogram on the left side (A). 

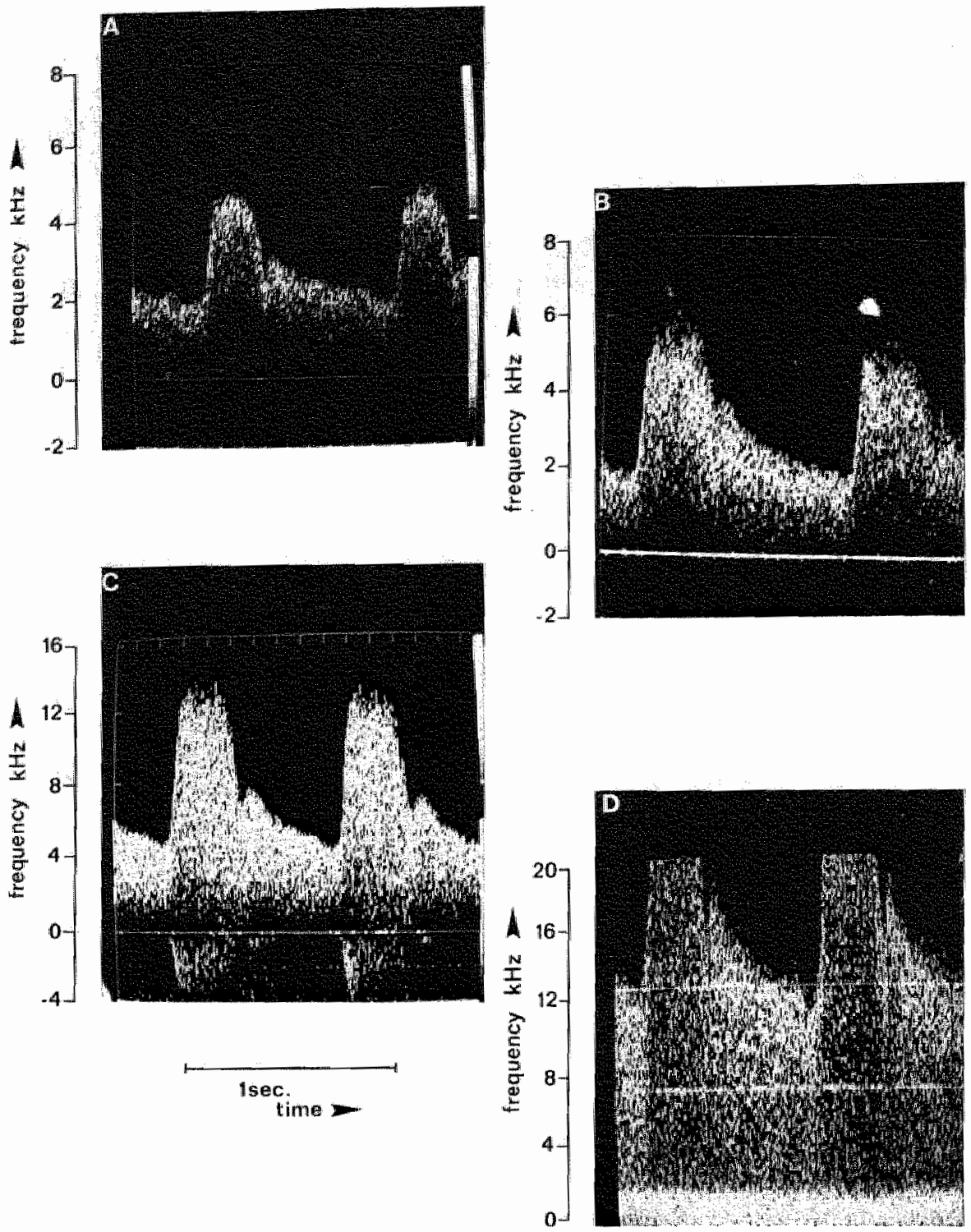

Figure 4-7. Sonograms of continuous-wave Doppler signals of internal carotid arteries, showing various degrees of spectral broadening. The degree of spectral broadening is classified as "Grade 1 " in sonogram (A); "Grade 2" in sonogram (B). "Grade 3" in sonogram (C) and "Grade 4 " in sonogram (D). In the sonograms (A), (B) and (C) the pattern of the internal carotid artery can be recognized. In sonogram (D) it is impossible to identify the artery from which the Doppler signal originates. The negative frequency shifts observed in the sonograms displaying moderate and severe spectral broadening "Grade 3 "and "Grade 4") represent the changes in blood flow velocties compatible with poststenotic rurbulence. 
If the direct Doppler examination is performed using a hand-held Doppler probe the peak systolic frequencies of the CCA and the ICA can only be compared bearing this limitation in mind.
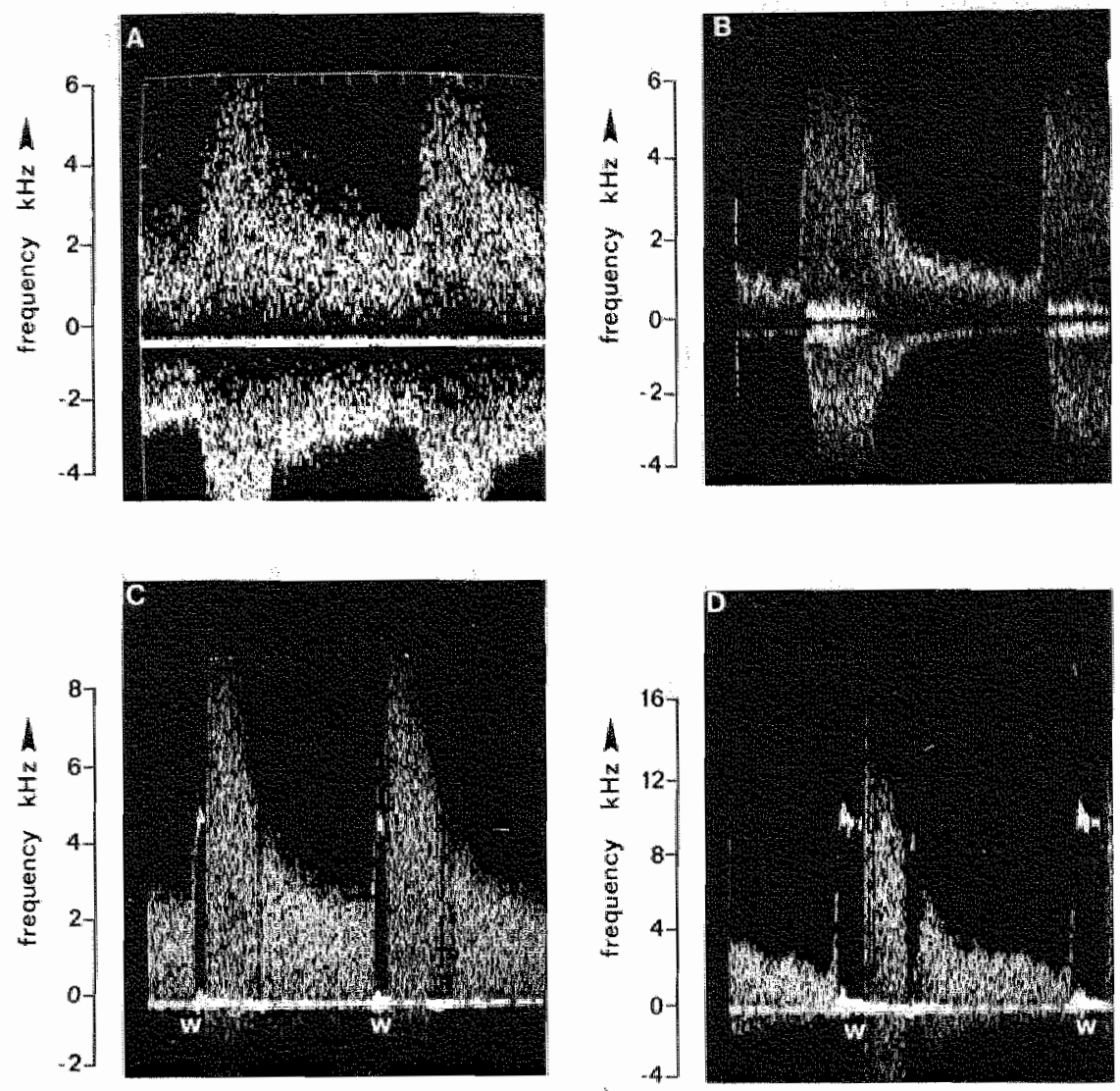

$1 \mathrm{sec}$

time

Figure 4-8. Sonograms of continuous-wave Doppler signals of internal carotid artcries and exrernal carotid arteries. The sonograms of the internal carotid artery $(A)$ and the external carotid artery (B) show mirroring of the Doppler signal and spectral broadening compatible with calcification of the arterial wall. The sonograms of the internal carotid artery (C) and the external carotid artery (D) show wall thumping (w), indicative of the highamplitude and low-frequency movement of the arterial wall. In these sonograms the upslope during systole is obscured because of the wall thumping. 


\section{4-2-3. Carotid phonoangiography}

In carotid phonoangiography a specially adapted microphone is placed along the course of the carotid artery in the neck. "The microphone is used to record sounds generated in the carotid arteries. These sounds are analyzed to detect bruits in this artery in relation to the first and second heart sound. The presence of a bruit is indicated by a noise pattern that masks normal heart sounds. Bruits may arise from the carotid artery, the great vessels in the thoracic cavity or the heart. The differentiation is facilitated by making recordings of at least three different levels i.e. over the CCA low in the neck, at the site of the carotid artery bifurcation, and over the ICA below the angle of the mandibula. Bruits detected low in the CCA are assumed to arise at or proximal to the origin of this artery. If the bruits are present at the mid or high cervical position they are considered to arise from the carotid artery bifurcation (133). The relative amplitude or intensity of the bruit along the course of the carotid arteries is probably not a good criterion for the source of the bruit as it primarely reflects the position of the microphone relative to the underlying artery (188). The relation of the bruit to the first and second heart sound and its duration are used for the estimation of the degree of stenosis of a lesion in the carotid artery bifurcation. There is an empiric relation between the duration of the bruit and the degree of stenosis $(134,189)$. Bruits that persist throughout systole and enter the diastolic phase are almost invariably associated with severe stenosis of the ICA. Bruits with a shorter duration indicate lesions with a lesser degree of stenosis. Sometimes bruits arising from the ICA and the ECA may be differentiated by their duration, as bruits from lesions in the ECA do not extend into diastole (189).

Although this technique is relatively simple and easy to learn it has its limitations. Not all stenotic lesions do cause a bruit since turbulence of sufficient energy is required to create an audible bruit (226). This implies mainly that bruits will be present when the lumen of the artery is narrowed substantially. Therefore, minor lesions cannor be detected by this technique. Furthermore, bruits often disappear in high grade stenoses [80\%-90\% diameter reduction] (161).

Based upon theoretical analysis of the production of bruits, secondary to flow disturbances at stenotic lestons, Lees and Dewey introduced spectral analysis of the bruit (162). Spectral bruit analysis relies on the theory that the more tight the stenosis is, the higher the break frequency will be. The break frequency is the frequency at which the intensity of the bruit begins to fall. The relationship between flow velocities through a stenosis and the break frequency of the bruit has been shown to be linear. Their data indicates that it is possible to estimate the degree of stenosis accurately, using this break frequency (66). However, the technique is on ly applicable to the relative small number of patients with a bruit, while those bruits arising from the ECA are a potential source of misinterpretation $(146,147,148,197)$. 
The indirect methods of investigation aim at the detection of changes in hemodynamics in the periorbital arteries, caused by a stenotic lesion in the ICA. These methods do not aim at the derection of flow disturbances, or the subsequent appearance of bruits at the site of the lesion, but try to record signs of a decreased pressure or a delayed pulse arrival secondary to the stenotic lesion as compared to a reference. These methods are called 'indirect' because they do not focus on the stenosis itself but are used at some distance from the lesion. Since the ophthalmic artery is the first major branch of the ICA the changes in pressure and flow in the orbital circulation may be sensitive indices of severe hemodynamic changes at proximal sites, such as the carotid artery bifurcation. The indirect tests may be divided into those aiming at the orbital branches of the ophthalmic artery ("superficial" tests) and those aiming at the bulbar branches of the ophthalmic artery ('deep' tests). The most commonly used technique for the investigation of the superficial orbital circulation is directional Doppler ultrasonography of the periorbital arteries. The other methods in this group include supraorbital photoplethysmography, thermography and supraorbital fluorescine resting $(81,82,118)$. Ocular pneumoplethysmography is the most commonly applied technique to investigate the deep orbital circulation; fundus reflectometry, oculotonography and ophthalmodynamometry are used less often $(13,111,258)$.

In fact, all these different techniques examine the same parameter: the pressure reduction as induced by a carotid artery stenosis. This parameter may be measured as an actual pressure reduction, or a change in the watershed between the distribution areas of the ICA and the ECA with subsequent changes in periorbital blood flow or a delayed pulse arrival. The limitations of the various indirect techniques are therefore identical. The indirect tests can only detect high grade lesions because only these lesions will cause a pressure drop. The different techniques that investigate the same part of the circulation, i.e. either the superficial orbital circulation or the deep bulbar circulation, cannot be expected to provide different informarion. Only those indirect methods of examination than are commonly used nowadays will be described.

\section{4-3-1 Oculoplethysmography according to Kartchner (OPG-K)}

The OPG-K was developed as a fluid filled system in which the transducers were coupled to the right and the left eye. The transducers record the cyclic variations in ocular volume. These variations are a reflection of the flow rate of blood into and from the eye during the cardiac cycle. The variations in ocular volume are not only recorded, but also compared electronically to detect small delays between the tracings that can not be detected by visual examination. This differential tracing is also recorded. To further facilitate the interpretation of the examination a transducer is coupled to the lobe of the ear to allow the comparison of pulse 
arrivals berween the eyes and the ear. The recordings are analyzed with regard to pulse arrival and the rate of the pulse increase. The presence of a significant lesion in the ICA is indicated by a delay in pulse arrival or a suppressed ocular pulse amplitude. $A$ unilateral stenosis is recognized by a delay in pulse arrival in comparison to the contralateral eye, or by a combination of pulse arrival delay and a decreased ocular pulse amplitude. According to Kartchner and McRae (136) the degree of the delay may be indicative of the severity of the stenosis. They were able to distinguish five different degrees of stenosis. Collateral blood flow has the effect of decreasing the pulse differences between the side of the stenosis and the contralateral side. Bilateral flow reducing stenoses of equal severity may be detected by bilateral delay with flattening of the ocular pulse or by a delay of 40 msec or more berween the pulse arrival time in the eyes and the pulse arrival time in the ear lobe (190).

The advantages of this technique are the applicability to virtually all persons in whom the eye is still present and the absence of an induced intraocular hypertension, thus minimizing the risks to the patients. Besides, glaucoma does not limit the performance and interpretation of the $O P G-K(190)$. The limitations of this technique are primarely related to the detection of bilateral stenoses. These lesions may not be detected as easily as suggested. Especially this aspect shows the observer variability in the interpretation of the OPG-K results $(28,185)$. Furthermore, the method provides only relative information. The OPG-K was developed simultaneously with carotid phonoangiography, by the same authors. In combination with carotid phonoangiography the test is consistently able to detect significant lesions in the carotid arteries $(30,37,135,140,143,241)$.

\section{4-3-2 Ocular pneumoplethysmography according to Gee (OPG-Gee)}

Ocular pneumoplethysmography (OPG-Gee) as available in its present form has been developed by Gee (88) by combining ocular plethysmography and suction ophthalmodynamometry $(43,87,261)$.

\section{4-3-2-1 Principle of the OPG-Gee}

The OPG-Gec enables the registration of the phasic changes in the wolume of the eye. These changes reflect the pulsarile arterial blood flow to the eye. Therefore, recording of the appearance of these volume changes may be indicative of the systolic pressure in the ophthalmic artery. In the OPG-Gee examination the pulsatile changes in the eye are recorded through an air-filled system of tubing and suction cup. The suction cup with a diameter of $14 \mathrm{~mm}$ has a smaller curvature than the bulb of the eye and, therefore, does not closely fit to the sclera. The air volume in the space between the suction cup and the sclera changes with the pulsations of the eye. These changes are picked up by a volume transducer and reconded for each eye separately. By means of restrictors in the volume transducers small non-phasic pulsatile changes are suppressed on the registration. 
Through the hollow tubing a negative pressure can be applied to the space berween the sclera and the suction cup. On a third tracing this applied negative pressure is recorded. The negative pressure maximally applied to the suction cups is $500 \mathrm{~mm} \mathrm{Hg}$. This negarive pressure can be gradually decreased to $0 \mathrm{~mm} \mathrm{Hg}$ in 32 seconds. By applying the negative pressure to the suction cup the geometry of the bulb of the eye is altered and the intraocular pressure increases $(86,269)$. The intraocular pressure may be increased to a level that exceeds the systolic blood pressure in the ophthalmic artery. This will result in the cessation of blood flow to the choroideal and retinal branches of the ophthalmic artery. With the gradual decrease of the applied negative pressure a point is reached when the intraocular pressure equals the systolic blood pressure in the ophthalmic artery. From that point on the orbital distension during the systolic phase of the cardiac cycle will cause the small portion of the sclera, drawn into the suction cup by the negative pressure applied, to be pulled out of the cup during systole. In the subsequent diastolic phase this part of the sclera will be pulled back into the suction cup. These pulsatile changes in the air volume between the sclera and the suction cup are transmitted to the volume transducer for recording. The appearance of the first pulsation on the recording reflects the height of the systolic blood pressure in the ophthalmic artery.

\section{4-3-2-2 Interpretation of the OPG-Gee}

The relationship between the negative pressure applied and the increased intraocular pressure has been established in experimental studies $(88,89,90)$. The conversion table for the applied negative pressure and the increased intracular pressure is shown in figure 4-9. An example of a recording of the OPG-Gee examination is shown in figure 4-10. The upper tracing shows the recording of the right eye and the lower tracing the recording of the left eye. In the middle tracing the decrease in the negative pressure is presented. The actual systolic pressure of the ophthalmic artery can be calculated by drawing a vertical line from the first pulsation of the right and left eye to the middle tracing, and converting the applied negative pressure at that point to the intracular pressure. In the presence of a hemodynamically significant stenosis in the ICA the pressure drop across the stenosis may be reflected by a lowered systolic pressure in the ophthalmic artery, as measured by the OPG-Gee. The criteria used for the interpretation of the $O P G-G e e$ are described in chapter 5. To allow a standard way of investigation the amplitudes of the plethysmograms can be calibrated.

The instrument is equipped with a fourth tracing, which is not shown in figure 4-10, to be used for the ECG recording. 


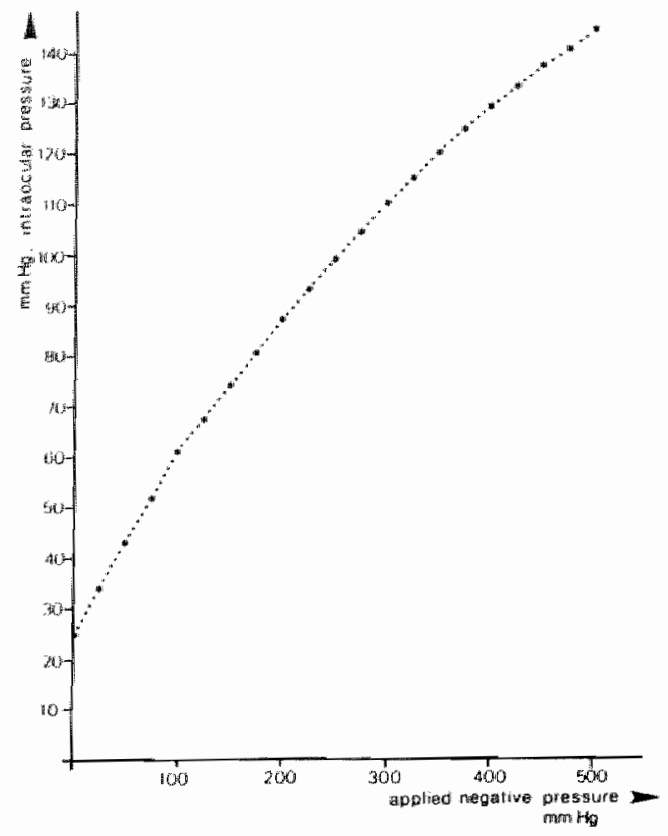

Figure 4-9. Diagram showing the ralation between the level of applied negative pressure in the suction cup of the ocular pneumoplethysmograpty according to Gee fon the horizonval axis) and the increase in intraocular pressure (on the vertical axis). The diagram is based on the findings of Galin er al $(86)$.

Figure 4-10. Recordings obtained at the ocular pneumoplethysmography examination according to Gee. The recordings show three tracings. The middle tracing shows the gradual decrease in the applied negatieve pressure. "The upper and the lower tracing show the registration of pulsations on the right and the lefteye, respectively.

In reording A the applied negative presure is $300 \mathrm{~mm} H \mathrm{Hg}(=5 \mathrm{~cm}$. After adecrease in the applicd negative pressure of $8 \mathrm{~mm}(=48 \mathrm{~mm} \mathrm{Hg}$ ) pulsations appear on the registrations of the right and the left eye. The applied negative pressure of $252 \mathrm{~mm} \mathrm{Hg}$ at that wime corresponds to an intracular pressure of $100 \mathrm{~mm} \mathrm{Hg}$. The systolic pressure in the ophthatmic artery (OA.P) on the right and the left side is $100 \mathrm{~mm} \mathrm{Hg}$.

In recording $B$ the applied negative pessure is $500 \mathrm{~mm} \mathrm{Hg}(-5 \mathrm{~cm})$. After a decrease of 5 mm ( $=50 \mathrm{~mm} \mathrm{Hg}$ pullsations appear on the registration of the left eyc. The applied negative pressure at the appearance of the arterial pulsations of $450 \mathrm{~mm} \mathrm{Hg}$ corresponds with an introcular pressure, and this an $O A P$, of $137 \mathrm{~mm} \mathrm{Hg}$. On the registration of the right eye the pulsations appear after a decrease in the applied negatuve pressure of $20 \mathrm{~mm}$ ( = $200 \mathrm{~mm} \mathrm{Hg}$. The intraocular pressure, and thus the $O A P$, at an applied negative pressure of $300 \mathrm{~mm} \mathrm{Hg}$ is $110 \mathrm{~mm} \mathrm{Hg}$. 

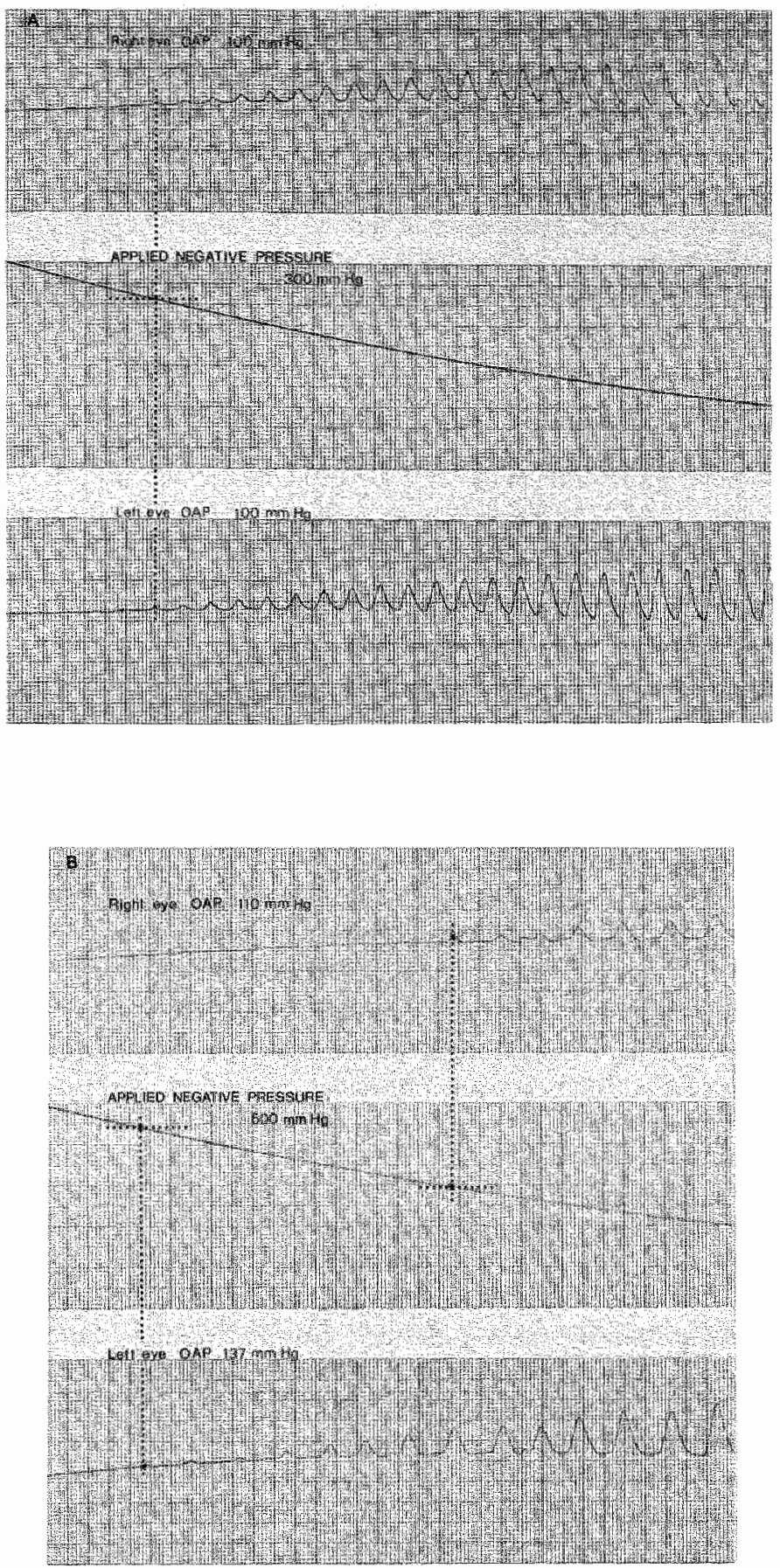
The OPG-Gee is a safe and simple indirecr method of examination for the evaluation of the carotid artery bifurcation. A vast experience with this technique has been gained by various investigators $(11,71,94,186,278)$. The test is well tolerated by the parients. If certain precautions (chapter 5 ) are respected the only complication reported of this examination is the appearance of posi-examination subconjunctival erythema or hematoma in approximately 3 per cent of the patients (11). The limitations of indireci tests, as discussed in chapter 4 , also apply to this test (95). Nevertheless, the OPG-Gee provides reliable information about the systolic ophthalmic artery pressure. An expert review of the possibilities and limitations of the OPG-Gee examination has been presented by Eikelboom (72).

In recent reports the combined use of the OPG-Gee and ocular pulse timing has been described $(12,27,245)$. Since this combination was not available at the time of our investigation it will nat be discussed here.

\section{4-3-3 The periorbital Doppler examination}

The periorbital Doppler examimation (OphthalmoSonoMetry = OSM) is the oldest cerebrovascular Doppler examination to idenrify hemodynamically significant obstructions in the ICA. The examination has been developed by Brockenbrough (44), who initially used a non-directional Doppler flowmeter. Subsequently, this test was refined by Maroon and colleagues (178) and Barnes et al (16).

\section{4-3-3- 1 Principle of the periorbital Doppler examination}

The examination aims at the detection of the direction of blood flow in the periorbital arteries and the response in these arteries to compression of the branches of the ECA. As already mentioned before (secrion 1-4-2) the periorbital arteries may act as a pathway of collateral circulation. Under normal circumstances the flow in the periorbital arteries is directed from the orbita to the forehead. Compression of the branches of the ECA (see below) will result in an increase of flow through the ophthalmic artery branches to compensate for the cessation of flow caused by the compression. Disturbances of the normal partern can be caused by an obstruction proximally in the ICA, causing a reduced pressure in the ipsilateral ophthalmic artery. This may result in diminished flow velocity, cessation of flow, or reversal of the direction of flow in the periorbital arteries. Even in the presence of a stenoric lesion in the ICA a normal direction of flow in the periotbital arteries may be seen after compression of the superficial temporal and the facial artery. The results of the OSM may be influenced by the presence of a stenotic lesion in the ECA or by intracranial arteriovenous fistulate (199). 
Blood flow in the periorbital arteries can easily be assessed with a Doppler thowmeter. The Doppler signals can be obtained from three periorbinal arteries: the frontal artery, the supraorbital artery and the dorsal nasal artery. The frontal artery, which is usually located near the inner canthus of the eye, runs with the supraorbital artery, which may be palpable in the supraorbital notch, to the forehead. The dorsal nasal artery runs below the medial canthus of the eye to the lateral aspect of the base of the nose. The dorsal nasal artery often shows reversed blood flow and may not communicate with the ophthalmic artery. The Doppler signal obtained over the frontal artery is usually most prominent and therefore most often used for the examination $(51,206)$.

\section{4-3-3-2 Interpretation of the periorbital Doppler examination}

Careful positioning of the Doppler probe just below the rim of the orbit is essential. The direction of flow in the periorbical arteries on both sides is established. Then the peak systolic frequency shift of the Doppler signal is measured in the sonogram of the frontal artery, at rest. Inadvertent reversal of the direction of blood flow may be obtained if the artery is insonated after it has crossed the orbital rim, where it runs away from the probe (203). Furthermore, the artery lies on a bony surface. The ultrasound may reach the artery after it has been reflected from the orbita, thus arousing the false impression of a reversal of the direcrion of flow (199). The compression manoeuvre is performed after localization of the superficial temporal artery, the facial artery and the infraorbital artery, at both sides. The superficial temporal artery is most easily compressed just above the zygoma, in front of the ear. The facial artery can be identified at the lower border of the mandibula, just in front of the masseter muscle; the infraorbital artery may be compressed at the site where it leaves the infraorbital foramen. After compression of the terminal branches of the ECA the direction of flow is, again, established. The criteria for the interpretation of the OSM are described in chapter 5.

\section{4-3-3-3 General remarks}

The OSM is a safe test and has been used for the evaluation of lesions of the ICA by many investigators $(36,65,138,199,201,279)$. The OSM is not a simple test. The positioning of the probe should be accurate and gentle. Interpretation of the examination may be hampered by the various pathways of collateral circulation and the backscatcering of the insonated beam of ultrasound from the underlying bone. As this test depends on the development of collateral circulation the presence of a stenoric lesion in the ECA, in combination with an ICA lesion may influence the interpretation of the test (170). The limitations of all indirect tests also apply to the OSM (section 4-3). The use of compression of the CCA, as advocated $(16,45)$, was not used in this study. In figure $4-11$ the normal response of the flow velocity in the frontal artery to compression of the branches of the ECA is shown. 


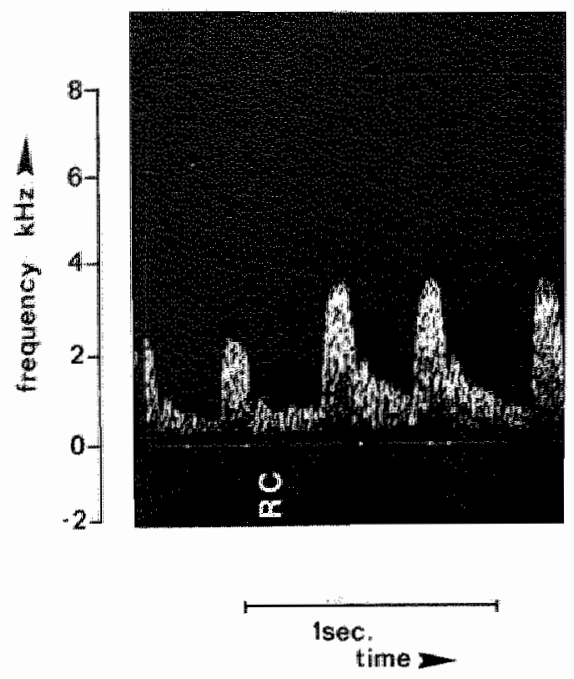

Figure 4-11. Sonogram of the continuous-wawe Doppler signal of the frontal artery. The sonogram shows the Doppler signal before and after compression of branches of the external carotid artery. The Doppler signals move from the left to the right. After the release of compression (R.C.) of the branches of the external carotid artery the peak systolic requency shifts decrease, indicating a decrease in blood llow velocity in the frontal artery from within the orbita outward.

\section{4-4 Contrast arteriography}

Contrast arteriography has been used for the documentation of the cerebral circulation since the report by Moniz in 1937 (200). In recent years the limitations of this technique as the "golden standard" for the evaluation of stenotic lesions in the carotid artery and the improved reliability of the noninvasive methods of examination have changed the central position of arteriography in the management of patients with cerebral ischemia.

\section{4-4-1 Technique}

In contrast arteriography of the cerebropetal and cerebral arteries an image of these arteries is obtained by the injection of a contrast medium in to this part of the arterial tree. The contrast medium is deposited with the catheter technique as described by Seldinger (246). In this technique a catheter is introduced over a guide wire by the transfemoral or transaxillary route into the ascending aorta and the carotid arteries selectively. Contrast arteriography performed in this way is a relatively safe procedure. The overall incidence of neurologic events following cerebral arteriography is approximately 1 to 2 per cent $(112,177)$. Most often transient neurologic abnormalities are seen, with stroke occurring in less than 0.1 
per cent of the patients (121). Complications related to the site of arterial puncture occur in 0.2 per cent in case of the transfemoral route and in 0.6 per cent in case of the transaxillary route (112).

In the performance of contrast arteriography certain limitations of this technique should be anticipated. Because obstructing atherosclerotic lesions frequently develop at arterial bifurcarions the demonstration of the luminal configuration in these junctional segments is particularly important. An atherosclerotic plaque in one branch may be obscured by the superimposed image of the other arterial branch. Furthermore, the stenotic lesion may be situated on the anterior or the posterior wall. If only one projection is used the lesion may not be evident. For these reasons two projections or more should always be made.

In the antero-posterior projection the superimposition of the dental fillings may be extremely bothersome, especially since the level of the carotid artery bifurcation may be quite variable. If the appearance of vasospasm is suspected it will often be induced by the catheter tip and the examination may have to be discontinued.

\section{4*4-2 Reliability of contrast arteriography}

Both interobserver and intraobserver variability in the estimation of the degree of stenosis of lesions shown on arteriography have been reported $(49,54,106)$. This may even become a limiting factor for the validation of noninvasive tests ( 150$)$. The reproducibility of the determination of the degree of stenosis can be improved by measuring (in $\mathrm{mm}$ ) rather than estimating the minimal diameter at the site of stenosis and the diameter of the 'normal' artery distal to the site of the lesion (47). DeWeese et al (62) found a "close" agreement between the measured percentage stenosis in gross specimen and on angiogram. A difference of less than $10 \%$ was found in 28 out of 34 patients $(82.4 \%)$. Croft $(57,58)$ found a "limited" agreement $(78 \%)$ between the X-ray interpretation and the findings at pathologic examination in a study on 64 dissected specimens of the carotid artery bifurcation. He defined "stenosis" only as a more than $20 \%$ lumern reduction, without further subdivisions.

The limitations of contrast arteriography and the variability in its interpretation create a problem in the assessment of the reliability of the noninvasive techniques in detecting stenotic lesions and their ability to quantify the severity of such lesions. Until now, however, no better "golden standard" is at hand.

\section{4-4-3 General remarks}

Bearing the limitations in mind $\mathrm{it}$ is imperative to review the images of the cerebral arteries obtained for technical imperfection while the catheter is still in place. If the examination requires an extension the subsequent images can be obtained with less effort and risks at this stage. Especially the possibility to add 
information by adjusting the angle of propection or performing cine-angiography has proved to be useful. If the noninvasive examination revealed stenotic lesions that were not suspected at arteriography, additional films were occasionally found to be helpful. Therefore, in the furure cerebral arteriography should only be performed if the radiologist has prior knowledge of the results of the noninvasive examination. All angiographies of the cerebropetal arteries as presented are reproduced from $X$-rays of patients included in the study. 


\title{
METHODS OF EXAMINATION
}

\author{
used in the present study
}

In this chapter the methods of investigation used in this study will be described. As the study was intended to compare the results of invasive and noninvasive methods of examination the criteria used for the interpretation of the different tests will be discussed.

\section{S-1 Contrast arteriography}

Because of the need to compare the results of the noninvasive tests with those of contrast arteriography only the patients with interpretable $\mathrm{X}$-rays of the carotid artery bifurcation and over 20 years of age entered the study. All arteriographic investigations were performed or supervised by a radiologist, with a vast experience in neuro-angiography.

\section{5-1-1 Technique of contrast arteriography}

Arteriography of the aortic arch was routinely performed in the $15^{\circ}$ left anterior oblique position, with a contrast medium administered in three seconds. Then the catheter could be exchanged, and positioned in the origin of the right and left CCA, respectively. Into each carotid artery $12 \mathrm{ml}$ of contrast medium was injected in one second. Simultaneous bi-plane views were made of both carotid arteries during the first seven seconds after injection, with multiple pictures per second during the first four seconds. Therefore, the CCA and its bifurcation could be examined in the antero-posterior projection and the lateral projection. Subsequently, the examination of the left vertebral artery and sometimes the right vertebral artery was performed. All pictures were examined by the radiologist during the examination. If the information as obtained from these projections was inadequate supplementary information was derived from X-ray films or cine-angiography following additional contrast injections. If necessary, conventional subtracrions of the arteriograms were provided for accurate determination of the degree of stenosis. 


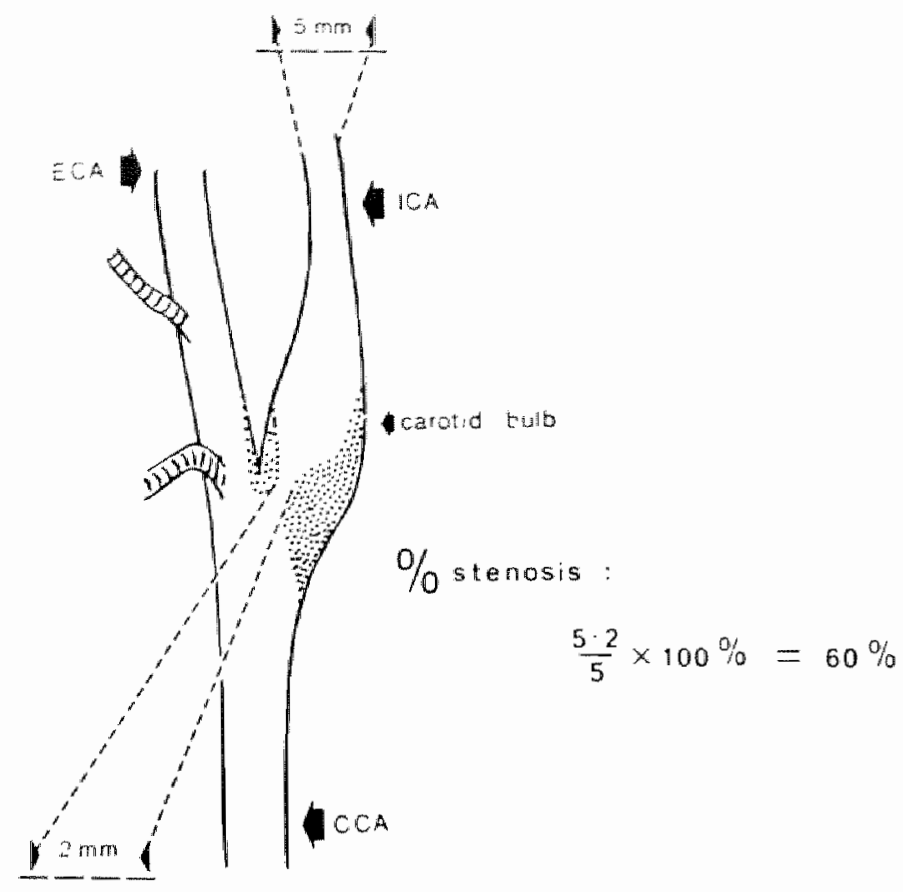

Figure 5-1. Diagram illustrating the measurement of the degree of stenosis of a lesion at the origin of the internal carotid artery.

\section{5-1-2 Interpretation of the arteriograms}

In the determination of the degree of stenosis of the carotid artery bifurcation the CCA, ECA and the ICA were considered separately. Stenotic lesions of the carotid artery arising at the carotid bilurcation and clearly compromising the origin of the ICA or the ECA were considered as lesions of the ICA or the ECA, respectively. Furthermore, the projection showing the stenotic lesion most clearly was used for the ultimate assessment of the severity of the lesion. The degree of stenosis was determined by expressing the difference between the smallest diameter at the site of stenosis and the diameter of the "normal" vessel at some distance from the stenosis as a percentage of this 'normal" diameter. In the ICA the measurement of the 'normal' diameter was performed distal to the carotid artery bulb. An example of the method of determination of the degree of 
stenosis is shown in figure 5-1. According to this procedure the degree of stenosis, in steps of $10 \%$, was determined independently by a radiologist and a surgeon. In case of disagreement in the estimation of the degree of stenosis the opinion of a third consultant was asked to reach a consensus.

Furthermore, the results of arteriography could be alloted to one of five categories, depending on the degree of stenosis. These categories, at forehand arbitrarily chosen, were:

$\begin{array}{llr}\text { I } & : & 0 \%-29 \% \text { stenosis } \\ \text { II } & : & 30 \%-49 \% \text { stenosis } \\ \text { III } & : 50 \%-69 \% \text { stenosis } \\ \text { IV } & : \quad 70 \%-99 \% \text { stenosis } \\ \text { V } & : \quad 100 \% \text { stenosis }\end{array}$

The resolution of cerebral arteriography under optimal conditions is $0.2 \mathrm{~mm}$, or more. Therefore, a differentiation between true negative arteriograms $10 \%$ stenosis) and arteriograms showing minimal lesions ( $1 \%$ to $9 \%$ stenosis) cannot be made. Even the classification of the degree of stenosis in steps of $10 \%$ is open for discussion.

At the time of this investigation digital subtraction angiography was not available in the hospital. Therefore, conventional arteriography, with occasionally subtractions of these arteriograms, was used throughout the study.

\section{5-2 The noninvasive examination}

The noninvasive tests were performed without prior knowledge of the results of the angiographic examination. The purpose of the examination was explained to the patient and an informed consent was obtained. The parient was asked for signs or symptoms of disorders that may interfere with the performance of the OPGGee examination. In this study the OPG-Gee examination was not performed if the patient had a history of retinal detachment, glaucoma, previous eye surgery, persistent eye infections, or when the patient could not deny such a condition, as in aphasia. Furthermore, the OPG-Gee was not performed if the patient was submitted to eye surgery in the near future.

The local anesthetic was installed on both eyes and cuff blood pressure measurements were performed on both upper arms, using a stethoscope. Both the systolic and diastolic brachial artery blood pressures were measured. The side with the highest blood pressure was taken as the reference for the OPG-Gee examination. Subsequently, the OPG-Gee examination, the periorbital Doppler examination and the direct Doppler examination of the cervical arteries were performed, in that order. All examinations were performed with the patient in the supine position. 


\section{5-2-1 Ocular pneamoplethysmography}

The OPG-Gee was performed after the patient had been resting at the vascular laboratory for approximately 10 minutes. The local anesthetic was installed as soon as the history of the patient, regarding contraindications for the OPG-Gee had been taken. The OPG-Gee was performed immediately after the measurement of the blood pressure.

\section{5-2-1-1 Method of examination}

In the OPG-Gee examination the pressure transducer was placed in front of the head of the patient, at such a distance that the connecting tubes between the eyecups and the transducer drooped and no tension was exerted to the eyes of the patient. The patient was warned that during the first few seconds after the maximal negative pressure had been applied loss of vision would occur, but that this loss would last only for seweral seconds. The cups were applied to the eyes, just lateral to the cornea. Under permanent surveillance the negative pressure was applied to the eye-cups. By starting the recording of the OPG-Gee the applied. negative pressure was gradually reduced. It is recommended to look at the recording during this procedure to detect artefacts, such as blinking of the eyes. immediately after this first recording a second examination was performed. If no useful] recording had been obtained, for example due to excessive eye movements, the procedure was started all over again. When adequate registrations were obrained the OPG-Gee examination was interpreted.

\section{5-2-1-2 Criteria for the interpretation of the OPG-Gee}

Since the introduction of the OPG-Gee several reports on the criteria used for the interpretation of this test have been published. Most commonly known are the criteria as developed by Gee (94), Eikelboom (72) and Machleder (10). In these criteria the relation between the systolic Brachial Artery Pressure (BAP) in the arm with the highest blood pressure and the systolic Ophthalmic Artery Pressure (OAP) is used.

\section{Criteria according to Gee:}

$=->$ for the detection of a unilateral hemodynamically significant lesion:

- both OAPs are above the lower limit of normal, as characterized by the formula $\mathrm{OAP}=39+0.43 \mathrm{BAP}$, and the OAP on the side of the lesion is at least $5 \mathrm{~mm} \mathrm{H} g$ lower than the contralateral OAP.

OR

- the OAP on the side of the hemodynamically patent ICA is above the lower limit of normal and the OAP on the side of the hemodynamically significant lesion is equal to or below the lower limit of normal, regardless the degree of difference in the OAPs. 
$=->$ for the detection of bilateral hemodynamically significant lesions:

- both OAPs are equal to or below the lower limit of normal. The OAPs may be equal, or different to a varying degree.

\section{Criteria according to Eikelboom:}

$=->$ for the detection of a unilateral hemodynamically significant lesion:

- a difference between the OAP of the right and left eye of $4 \mathrm{~mm} \mathrm{Hg}$ or more OR

- a difference in the amplitudes of the recorded signal of the right and the left eye of $2 \mathrm{~mm} \mathrm{Hg}$ or more, when both OAPs exceed the maximally induced intra-ocular pressure.

$=->$ for the detection of bilateral hemodynamically significant lesions:

- both $O A P s$ are less than the maximally induced intraocular pressure and less than $60 \%$ of the BAP.

\section{Criteria according to Macbleder:}

$=->$ for the detecrion of a unilateral hemodynamically significant lesion:

- a difference in the OAPs of the right and left eye of $5 \mathrm{~mm} \mathrm{Hg}$ or more

$\mathrm{OR}$

- a difference in the OAPs of the right and left eye of 1 to $4 \mathrm{~mm} \mathrm{Hg}$ and a ratio of the lowest OAP and the highest BAP of less than 0.66: (OAP / BAP <0.66).

$=->$ for the detecrion of bilateral hemodynamically significant lesions:

- no OAP asymmetry, but a ratio of the lowest OAP and the highest BAP of less than 0.60: (OAP / BAP <0.60).

The BAP, and therefore, the OPG-Gee can only be used if the Doppler examination of the brachial artery shows a normal signal. Each of the authors considers the OPG-Gee to be indicative of a stenotic lesion in the ICA if one of the criteria is met.

\section{5-2-2 The Doppler examinations}

Two of the noninvasive tests concerned CW Doppler examinations of the extracranial cerebropetal arteries. In these examinations the Doppler signal was processed by the UNIGON ANGIOSCAN*. Some features of this instrument will be described below.

\section{5-2-2-1 The Doppler instrumentation}

The examinations were performed with a bi-directional CW Doppler flowmeter (MEDASONICS $D^{9}$ ). The cervical arteries were insonated with an $8 \mathrm{MHz}$ Doppler probe. An $8 \mathrm{MHz}$ probe was chosen because of its adequate penetration and high frequency resolution (235). If necessary a $5 \mathrm{MHz}$ probe could be used. The Doppler signal was processed with the use of a UNIGON ANGIOSCAN* spectral analyzer based upon Fast Fourier Transform. The instrument displayed the sonogram on a $23 \mathrm{~cm}$ (diagonal) video screen. The frequency shift was shown along the vertical axis and the time was displayed horizontally. The amplitude of 
the frequencies was displayed as shades of gray. The sonogram moved from the left to the right on the screen and the time axis could be extended, by choice, for $1.6,3.2$ or 9.6 seconds. The image presented on the screen could be frozen indefinitely, either by hand or foot switch. The frequency range displayed on the full screen could be set from $5 \mathrm{kHz}$ to $20 \mathrm{kHz}$ to allow measurements over a wide range of frequency shifts. A five position input level switch provided sensirivities covering a range from $100 \mathrm{mV}$ to $10 \mathrm{~V}$, to accept the output from most commercially available Doppler flowmeters. A light emission diode (LED) inpuz level indicated the input overload. A $300 \mathrm{~Hz}$ high pass filter could be switched "on" or "off" to eliminate the already described artifacts due to excessive vessel wall thumping. The instrument as equiped with a cursor, consisting of a horizontal line, which could be moved up and down the display. The frequency indicated by the cursor was digitally presented on the screen. In later models the screen allowed the display of patient identification and the description of the vessel under investigation, while also certain parameters of the sonogram could be displayed. The instrument used in this study did not yet provide hard copy documentation and, therefore, self-developing photographs of the video screen had to be taken. The sonograms, as presented in this study, were reproduced from them. The characteristics of the instrument were described by Macpherson et al (174).

\section{5-2-2-2 The periorbital Doppler examination}

The OSM was performed after the OPG-Gee had been interpreted, allowing the patient some time to adjust after the OPG-Gee examination.

\section{5-2-2-2-1 Method of examination}

For this examination the patient was asked to close the eyes and acoustic gel was applied to the inner canthus of the upper eyelid. Using an $8 \mathrm{MHz}$ bi-directional CW Doppler probe, the frontal and the supraorbital arteries were located and insonated. The artery with the strongest Doppler signal was used for the examination. The Doppler probe was carefully positioned below the orbital rim to ensure correct interpretation of the direction of flow in the periorbital artery. The Doppler signal obtained from the periorbital artery was recorded. Then the compression manoeuvre was started by compressing the superficial temporal artery and the facial artery on the same side. The change in the Doppler signal, as far as peak systolic velocity and direction of flow are concerned, was recorded. Subsequently, the superficial temporal artery and the tacial artery on the contralateral side were compressed and again the changes in the Doppler signal were recorded. The changes in the Doppler signal of the periorbital artery following compression of the foramen infraorbitale were investigated. If still doubt existed about the periorbital Doppler examination the procedure was repeated with combined compression of all arteries. The same procedure was followed on the other side. 
The use of a bi-directional Doppler flowmeter permits the identification of the direction of flow. The test was considered indicative of a hemodynamically significant lesion in the ICA if one of the following criteria was met:

$\rightarrow \rightarrow$ reversal of the normal direction of blood flow in the periorbital arteries.

$\rightarrow \rightarrow$ diminution or absence of blood flow velocity in the periorbital arteries following compression of the branches of the ECA. The branches of the ECA may be compressed separately on the ipsilateral or contralateral side, or simultaneously.

$=->$ a difference in the peak systolic frequency shift berween both sides of $40 \%$ or more.

The first and second criterion can be used for the detection of bilateral obstructions in the ICA, while the third criterion can only be used to identify a lesion on the side with the lower frequency shift.

\section{5-2-2-3 The direct Doppler examination}

Subsequently the direct Doppler examination was performed, after the acoustic gel had been removed from the upper eye lid.

\section{5-2-2-3-1 Method of examination}

In this examination the head of the patient was slightly extended and rotated away from the side of investigation. The examination was performed with an $8 \mathrm{MHz}$ CW bi-directional Doppler probe. The CCA was insonated low in the neck and followed until the bifurcation was reached. Then the ECA was followed. If the ECA had been followed for approximately two centimeters the ICA could be found by moving the Doppler probe slowly outward. The ICA may be located just lateral and dorsal to the ECA. The differences in the Doppler signals of the ECA and the ICA can be appreciated from both the audible and the visual information provided to the examiner. If srill doubr existed, as might be the case if severe stenotic lesions were present in both the ECA and the JCA, intermittent compression of the superficial temporal artery often yielded additional information. The carotid artery bifurcation was examined more closely by approaching it from the three different vessels. The various arteries were then insonated. The angle between the Doppler probe and the vessel was unknown. The position of the probe was adjusted to produce recordings with the highest peak frequency, the least amoun of spectral broadening at peak systole, the maximum power, and the best signal - to - noise ratio. Only after it had been established that the findings in the three vessels were reproducible the examination was completed by recording the characteristics of the vessels under examination. Subsequently, the same procedure was followed on the other side. 
The carotid arteries were insonated repeatedly and from different angles. Whether a lesion was present in one of the arteries was decided during the investigarion. The direct Doppler examination was performed in approximately 35 minutes (range 23 minutes to 45 minutes). The maximal frequency shifts in the CCA, the ECA and the ICA were compared and local increases in the peak systolic frequency were sought for. The maximal peak systolic velocities were recorded. If the direct Doppler examination was performed with a $5 \mathrm{MHz}$ rather than an $8 \mathrm{MHz}$ emission probe the maximal peak frequency recorded was multiplied by $8 / 5$ to correct for the difference in emission frequency. Of course, $8 / 5$ is only an approximation of the ratio of the emission frequencies. The degree of spectral broadening was estimated. For clinical use the interpretation of the sonogram can be based on the presence of a clear area (the 'window'; "Grade 1 "), or the presence of spectral broadening in the Doppler signal ("Grade 2", or "Grade 3", or "Grade 4") or absence of the Doppler signal ("Grade 5"). The "Grade 2" sonograms showed spectral broadening, without negative frequency shifts, and the pattern of the sonogram could be recognized as arising from either the CCA, the ECA or the ICA. Only if the spectral broadening was associated with the presence of negative frequency shifts and the pattern of the sonogram could still be recognized as arising from a specific artery the sonogram was interpreted as "Grade 3". If the Doppler signal of the sonogram could not be interpreted as arising from the CCA, the ECA or the ICA and the sonogram showed severe spectral broadening it was interpreted as "Grade 4", assuming to arrive from the ICA. In the absence of a Doppler signal the artery was supposed to be occluded ("Grade 5"). It is obvious that this visual interpretation of the sonogram remains a personal impression of the degree of spectral broadening. This subjective approach has been used by a variety of investigators $(4,18,19,78)$ because accurate quantification of the degree of spectral broadening of the Doppler signal is still problematic $(64,130,137,150,235)$. The appearance of the 'window' is influenced by the signal-noise ratio. Therefore, to allow a fair interpretation of the sonograms the input sensitivity of the amplitude of the Doppler signal should be set, and maintained at that level. The sonograms of the carotid arteries were allotted to one of 5 categories, describing various degrees of spectral broadening. These categories were used by Barnes and collaborators (19). They defined these categories as follows:

Grade I : normal spectral distribution with concentration of the intensity of the spectrum along the upper border of the frequency envelope.

Grade II : mild spectral broadening with an increase of amplitudes in the lower frequency domain, while maintaining the major portion of the amplitudes near the upper frequency. 
Grade III : moderate spectral broadening with equal amplitudes of signals throughout the range of frequencies.

Grade IV : severe spectral disturbance with the majority of the signal amplitudes in the lower frequency range.

Grade V : absence of Doppler signals, implying occlusion of the vessel.

In all cases the decision to allot the result of the direct Doppler examination to one of the five categories of spectral broadening was made at the end of the examination for each of the investigated arteries. Then the degree of spectral broadening was assessed and the result was allotted to one of the five categories. The personal interpretation of the sonogram was documented on self-developing films. Examples of the sonograms typical for the various degrees of spectral broadening of the ICA are shown in figure 4-5.

Bandyk et al (14), Barnes (19) et al and Arbeille et al (4) suggested that the classification of the degree of spectral broadening of the Doppler signal could be related to the degree of stenosis found at arteriography. In this study we attempted to relate the five categories of stenosis at arteriography, as mentioned in section 5-1-2, to the classification of spectral broadening as is shown in table $5-1$.

TABLE 5-1. Assumed correlation of the degree of spectral broadening of the Doppler signal of the ICA and the severity of the stenosis of the ICA as seen at arteriography.

degree of

stenosis:

Grade 1

--- degree of spectral broadening ---

0. $29 \%$

$\mathrm{X}$

30. $49 \%$

$50-69 \%$

$70-99 \%$

$\mathrm{X}$

$100 \%$

$\mathrm{X}$

\section{5-3 Statistical analysis}

The results of the noninvasive tests in comparison to the results of arteriography of the extracranial cerebropetal arteries can be described as the ability of the noninvasive tests to detect stenotic lesions of these arteries, or their ability to exclude such lesions.

The use of the sensitivity and the specificity of a noninvasive test enables the comparison of the results of that noninvasive test with other tests and studies. These values are not influenced by the prevalence of the disease in the group of patients under investigation, in contrast to the diagnostic accuracy, the positive predicrive value and the negative predictive value. The decision matrix from which these values can be calculated is shown in table 5-2. 
TABLE 5-2. Decision matrix for the determination of sensitivity, specificiry, diagnostic accuracy, positive predictive value and the negative predicrive walle.

ARTERIOGKAHYY $(+)$ ARTERTOGRAPHY $(-)$ TOTAL

$\begin{array}{lccc}\text { TEST" }(+) & \mathrm{A} & \mathrm{B} & \mathrm{A}+\mathrm{B} \\ \text { TEST }(-) & \mathrm{C} & \mathrm{D} & \mathrm{C}+\mathrm{D} \\ \text { TOTAL } & \mathrm{A}+\mathrm{C} & \mathrm{B}+\mathrm{D} & \mathrm{T}\end{array}$

sensitivity

specificity

diagnostic accuracy

posiche predicrive value

negative predictive walue
$A /(A+C) \times 100 \%$

$D /(B+D) \times 100 \%$

$: \quad(A+D) / T \times 100 \%$

$: A /(A+B) \times 100 \%$

: $D /(C+D) \times 100 \%$

Obviously, a good diagnostic test has a good sensitivity and a good specificity. The interpretation of the results of the various noninvasive tests is facilitated by plotting the sensitivity and 1-specificity against each other. The plot, which takes the shape of a concave curve, is known as a receiver-operating-characteristic curve, "ROC curve" (187).

The diagnostic accuracy of a test depends on the prevalence of disease in the population under investigation (208). The Kappa-corrected diagnostic accuracy allows better comparison of the results of different noninvasive studies because it corrects the diagnostic accuracy for differences in the composition of the population under investigation $(244,256)$. Kappa-corrected diagnostic accuracy can be thought of as observed agreement in the interpretation of the test compared to the golden standard not accounted for by chance, divided by the possible agreement not accounted for by chance (152). Like a correlation coefficient Kappa-corrected diagnostic accuracy varies fron -1.0 (complete disagreement) through 0 (chance agreement) to +1.0 (perfect agreement). The Kappa-corrected diagnostic accuracy is characterized by the formula: Kappa $=(\mathrm{Po}-\mathrm{Pc}) /(1-\mathrm{Pc})$, in which $\mathrm{Po}=$ the observed proportion of agreement and $P_{C}=$ the proportion of agreement expected by chance. Kappa can be calculated as shown in table 5-3. 
TABLE 5-3. Example of the derermination of the Kappa-corrected diagnostic accuracy -- degree of spectral broadening -..-

stenosis Grade 1 Grade 2 Grade 3 Grade 4 Grade 5 total

$\begin{array}{rrrrrrr}0 \%-29 \% & 168 & 12 & 0 & 0 & 1 & 181 \\ 30 \%-49 \% & 2 & 22 & 2 & 0 & 0 & 26 \\ 50 \%-69 \% & 0 & 0 & 18 & 3 & 0 & 21 \\ 70 \%-99 \% & 0 & 2 & 12 & 32 & 0 & 46 \\ 100 \% & 0 & 0 & 0 & 2 & 20 & 22 \\ \text { total } & 170 & 36 & 32 & 37 & 21 & 296\end{array}$

Kappa $=\left(\mathrm{Po}_{\mathrm{O}} \mathrm{Pc}\right) /(1-\mathrm{P} \mathrm{c})$, in which:

$\mathrm{P}_{\mathrm{O}}=$ observed proportion of agreement $=(168+22+18+32+20) / 296=0.88$

$\mathrm{P}_{\mathrm{c}}=$ proportion of agreement by chance: $=$

$[(181 / 296) \times(170 / 296)]+$

$[(26 / 296) \times(36 / 296)]+$

$[(21 / 296) \times(32 / 296)]+$

$[(46 / 296) \times(37 / 296)]+$

$[(22 / 296) \times(21 / 296)]+=0.39$

Kappa $=(0.88-0.39) /(1-0.39)=0.49 / 0.61=0.80$ 


\section{RESULTS}

\section{6-1 Description of the patients}

In the present study 148 consecutive patients in whom cerebral arteriography was performed were investigated, between may 10, 1981 and december 31, 1982. All patients were selected for cerebral arteriography by the neurologist. The decision to perform cerebral arteriography was based on clinical symptoms of ischemic and non-ischemic cerebral dysfunction. Therefore, this series includes patients without atherosclerotic stenotic lesions of the cerebropetal arteries. The indication to perform cerebral arteriography and the correlation of symptoms and the findings of arteriography were no subject of this study. In this series 52 female patients (ages ranging from 23 to 76 years, mean age 59.2 years) and 96 male patients (ages ranging from 35 to 76 y ears, meara age 59.7 years) were investigated. The distribution of sex and age per decade is shown in figure 6-1.

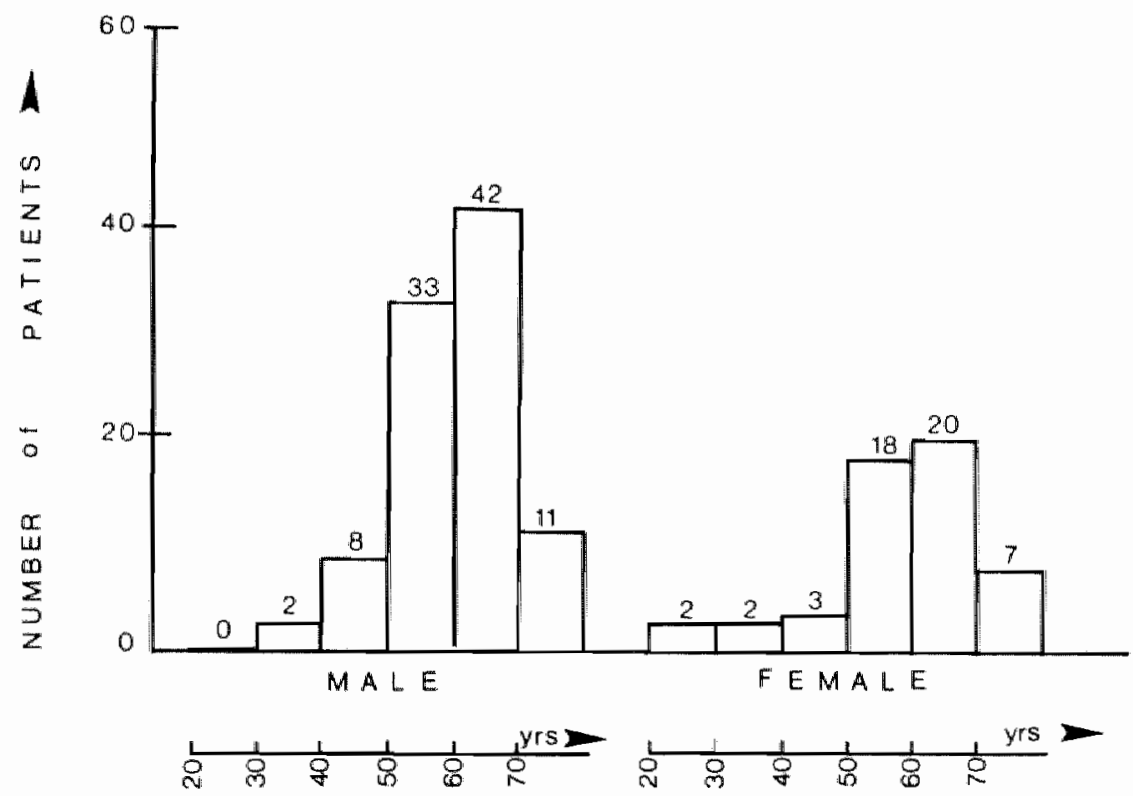

Figure 6-1. Diagram showing the number of male and female patients in the various decades. 
A history of reversible or permanent cerebral ischemia was present in 25 of the 52 female patients, and in 50 of the 96 male patients. Besides, 17 of the 52 female patients and 34 of the 96 male patients had a history of coronary artery disease. A history of both cerebral ischemia and coronary artery disease was present in 7 of the female patients and in 13 of the male patients. Of the 148 patients 82 had the habit of smoking. The prevalence of cerebral ischemia and coronary artery disease in relation to smoking is shown in table 6-1 for the female and in table 6-2 for the male patients. In two of the patients it was impossible to obtain adequate information about the history of cerebral ischemia and in one of the patients it was impossible to obtain information concerning symptoms of coronary artery disease, cerebral ischemia or his habit of smoking.

TABLE 6-1. Prevalence of coronary artery disease (CAD) and cerebral ischemia (CI) in female patients, in relation to smoking.

\begin{tabular}{|c|c|c|c|c|c|c|}
\hline & \multicolumn{3}{|c|}{$\mathrm{CAD}(+)$} & \multicolumn{3}{|c|}{$\mathrm{CAD}(-)$} \\
\hline & $\mathrm{Cl}(+)$ & $\mathrm{CI}(-)$ & $\mathrm{CI}(?)$ & $\mathrm{Cl}(+)$ & $\mathrm{Cl}(-)$ & Cll? \\
\hline smoking $(+)$ & 4 & 6 & - & 5 & 4 & - \\
\hline smoking $(-)$ & 3 & 4 & - & 13 & 12 & 1 \\
\hline
\end{tabular}

TABLE 6-2. Prevalence of coronary artery disease (CAD) and cerebral ischemia (CI) in male patients, in relation to smoking.

\begin{tabular}{lcccccc} 
& \multicolumn{3}{c}{$\mathrm{CAD}(+)$} & \multicolumn{3}{c}{$\mathrm{CAD}(-)$} \\
& $\mathrm{Cl}(+)$ & $\mathrm{Cl}(-)$ & $\mathrm{Cl}(?)$ & $\mathrm{Cl}(+)$ & $\mathrm{CI}(-)$ & $\mathrm{Cl}(?)$ \\
smoking (+) & 7 & 14 & - & 22 & 19 & 1 \\
smoking (-) & 6 & 7 & - & 15 & 4 & - \\
\hline
\end{tabular}

\section{6-2 Results of arteriography}

A prerequisite of this study was that the arteriograms of the cerebropetal arteries were of sufficient quality to allow interpretation of the cxtracranial cerebral arteries and the intracranial part of the ICA. This condition was met in 148 patients, bilaterally. Therefore, a total of 296 segments of the cerebropetal arteries were available for interpretation and comparison.

6-2-1 Arteriographic lesions in the proximal part of the brachiocephalic arteries At arteriography 44 stenotic lesions of the proximal part of the brachiocephalic arteries were found in 30 parients. These stenotic lesions were located in the innominate artery in 5 cases, and in the right and the left CCA in 5 cases and 6 
cases, respectiwely. A stenosis of a subclavian artery was found in 20 cases. The vertebral artery showed a stenosis in 8 cases. The prevalence and degree of stenosis of the lesions in the proximal part of the brachiocephalic arteries are shown in table 6-3.

TABLE 6-3. Prevalence and degree of stenosis of lesions in the proximal part of the brachiocephalic arteries in 148 patients.

\begin{tabular}{|c|c|c|c|c|c|c|c|}
\hline \multirow{2}{*}{$\begin{array}{l}\text { degree of } \\
\text { stenosis }\end{array}$} & \multirow[t]{2}{*}{$\begin{array}{c}\text { invominate } \\
\text { artery }\end{array}$} & \multicolumn{2}{|c|}{$\begin{array}{c}\text { common carotid } \\
\text { artery }\end{array}$} & \multicolumn{2}{|c|}{$\begin{array}{c}\text { vertebral } \\
\text { artery }\end{array}$} & \multicolumn{2}{|c|}{$\begin{array}{c}\text { subclavian } \\
\text { artery }\end{array}$} \\
\hline & & $\mathrm{R}$ & $L$ & $\mathrm{R}$ & $L$ & $\mathrm{R}$ & L \\
\hline $0-9 \%$ & 143 & $\{43$ & 142 & 145 & 143 & 137 & 139 \\
\hline $10-19 \%$ & 1 & 1 & 2 & - & - & $-\cdots$ & 1 \\
\hline $20-29 \%$ & 1 & 1 & 2 & - & - & $\mathbb{1}$ & - \\
\hline $30-39 \%$ & - & 1 & 1 & - & $\mathbb{1}$ & - & - \\
\hline $40-49 \%$ & 1 & - & - & - & $\mathbb{1}$ & 3 & 1 \\
\hline $50-59 \%$ & - & - & - & j & $\mathbb{1}$ & 1 & 1 \\
\hline $60-69 \%$ & - & - & - & 1 & $\sim$ & 3 & 1 \\
\hline $70-79 \%$ & 1 & - & 1 & - & 2 & - & 3 \\
\hline $80-89 \%$ & - & 2 & - & - & - & 1 & 1 \\
\hline $90-99 \%$ & - & - & -- & - & - & 2 & - \\
\hline $100 \%$ & 1 & - & - & 1 & - & - & 1 \\
\hline total & $\llbracket 48$ & 148 & 148 & 148 & 148 & 148 & 148 \\
\hline
\end{tabular}

6-2-2 Arteriographic lesions at the origin of the external carotid artery

In 35 patients a stenotic lesion was found at the origin of the ECA. In 12 of these patients both ECAs were involved. The prevalence and degree of stenosis of the lesions found at the origin of the ECA are shown in table 6-4.

TABLE 6-4. Prevalence and degree of stenosis of lesions at the origin of the ECA in 148 patients (296 sides).

$\begin{array}{lrrr}\text { degrec of } & \text { right ECA } & \text { 1cfr ECA } & \text { toral } \\ \text { stcnosis } & & & \\ 0-9 \% & 120 & 129 & 249 \\ 10-19 \% & - & - & - \\ 20-29 \% & 1 & - & 1 \\ 30-39 \% & 1 & 1 & 2 \\ 40-49 \% & 4 & 3 & 7 \\ 50-59 \% & 4 & 3 & 7 \\ 60-69 \% & 5 & 2 & 7 \\ 70-79 \% & 1 & 2 & 3 \\ 80-89 \% & 6 & 6 & 12 \\ 90-99 \% & 3 & 1 & 4 \\ 100 \% & 3 & 1 & 4 \\ \text { total } & 148 & 148 & 296\end{array}$


In 25 of the 28 cases with a stenosis of the tight ECA a stenotic lesion was present in the ICA at the same side. In 2 of the 3 cases in which the lesion in the right ECA was not associated with a lesion in the right ICA a stenosis of the left ICA was found. At the left side a stenosis in the ICA was found in 16 of the 19 cases with a lesion in the ECA. If the lesion in the ECA was not associated with a lesion in the ICA at the same side, again a contralateral lesion in the ICA was found in 2 cases.

\section{6-2-3 Arteriographic lesions at the origin of the internal carotid artery}

Angiographic examination of the carotid artery bifurcation showed a lesion with a degree of narrowing of $10 \%$ or more in 155 ICAs. The prevalence and degree of stenosis of the lesions at the origin of the ICA are shown in table 6-5.

TABLE 6-5. Prevalence and degree of stenosis of lesions at the origin of the ICA in 148 parients (296 sides).

\begin{tabular}{lrrr}
\hline degree of & right ICA & lefi ICA & rotal \\
stenosis & & & \\
$0-9 \%$ & 70 & 71 & 141 \\
$10-19 \%$ & 8 & 12 & 20 \\
$20-29 \%$ & 10 & 10 & 20 \\
$30-39 \%$ & 3 & 3 & 6 \\
$40-49 \%$ & 13 & 7 & 20 \\
$50-59 \%$ & 2 & 5 & 7 \\
$60-69 \%$ & 8 & 6 & 14 \\
$70-79 \%$ & 4 & 5 & 9 \\
$80-89 \%$ & 8 & 5 & 13 \\
$90-99 \%$ & 10 & 14 & 24 \\
$100 \%$ & 12 & 10 & 22 \\
total & 148 & 148 & 296 \\
\hline
\end{tabular}

At arteriography elongation of the ICA was seen in 15 arteries. In three of these vessels kinking of the ICA was found at arteriography.

6-2-4 Arteriographic lesions in the intracranial part of the internal carotid artery In the intracranial part of the [CA a stenotic lesion was seen in 15 patients. In 5 of these patients a bilateral lesion was found. The severity of these lesions is shown in table 6-6. In 3 sides the lesion in the ICA syphon was not associated with a lesion at the origin of the ipsilateral ICA. The degree of stenosis of these 'solitary' lesions was $10 \%, 10 \%$ and $40 \%$, respectively. In 6 sides ( 4 patients) the syphon lesion was more severe than the lesion at the origin of the ICA. 
TABLE 6-6. Prevalence and degree of stenosis of lesions in the intracranial part of the TCA in 148 patients (296 sides).

\begin{tabular}{|c|c|c|c|}
\hline $\begin{array}{l}\text { degree of } \\
\text { stenosis }\end{array}$ & $\begin{array}{l}\text { syphon } \\
\text { right ICA }\end{array}$ & $\begin{array}{l}\text { syphon } \\
\text { left ICA }\end{array}$ & total \\
\hline $0-9 \%$ & 136 & 140 & 276 \\
\hline $10-19 \%$ & 4 & 4 & 8 \\
\hline $20-29 \%$ & 3 & 1 & 4 \\
\hline $30-39 \%$ & - & 1 & $\Downarrow$ \\
\hline $40-49 \%$ & 2 & - & 2 \\
\hline $50-59 \%$ & 1 & - & 1 \\
\hline $60-69 \%$ & 2 & - & 2 \\
\hline $70-79 \%$ & - & 2 & 2 \\
\hline $80-89 \%$ & - & - & - \\
\hline $90-99 \%$ & - & - & - \\
\hline $100 \%$ & - & - & - \\
\hline rotal & 148 & 148 & 296 \\
\hline
\end{tabular}

\section{6-2-5 Description of the 'arterial segments'}

The direct Doppler examination of the cervical carotid arteries can be expected to detect stenotic lesions within reach of the emitted ultrasonic beam. Therefore, the results of the direct Doppler examination will be compared with the results of arteriography as shown in table 6-4 for the ECA and in table 6-5 for the ICA. On the other hand, the indirect noninvasive tests can be expected to reflect the hemodynamics of the cerebropetal circulation between the aortic arch and the branches of the ophthalmic artery under investigation. Therefore, to allow a fair comparison of the results of the different noninvasive tests with those of arteriography the latter have to be described per bifurcation for the direct noninvasive test but per arterial segment, including the entire parhway from the aortic arch to the ophthalmic artery, for the indirect noninvasive tests. The results of the indirect noninvasive tests will be compared to the results of arteriography as shown in table 6-7 describing the most severe lesion, i.e. the lesion with the most severe degree of narrowing, in the segment of the cerebropetal circulation under investigation. The hemodynamic effects of multiple lesions of a lesser degree of narrowing may be more pronounced than those of one severe lesion alone. This aspect has not been solved and, therefore, may influence the overall results as obtained with the indirect tests.

A stenotic lesion in the proximal part of the brachiocephalic arteries or in the syphon of the ICA, was more severe than the stenosis at the origin of the ICA in 11 patients. In 2 of these patients this situation was met bilaterally. The degree of stenosis per side studied, including the more proximally and distally located lesions, is shown in table $6-7$. 
TABLE 6-7. Prevalence and degree of stenosis of the most severe lesion in the arterial segments from the aortic arch to the ophthalmic artery in 148 patients $(296$ sides).

\begin{tabular}{lrrr}
\hline $\begin{array}{c}\text { degree of } \\
\text { stenosis }\end{array}$ & right side & left side & rotal \\
$0-9 \%$ & 66 & 70 & 136 \\
$10-19 \%$ & 9 & 11 & 20 \\
$20-29 \%$ & 9 & 10 & 19 \\
$30-39 \%$ & 3 & 3 & 6 \\
$40-49 \%$ & 13 & 6 & 19 \\
$50-59 \%$ & 2 & 5 & 7 \\
$60-69 \%$ & 8 & 7 & 15 \\
$70-79 \%$ & 5 & 7 & 12 \\
$80-89 \%$ & 10 & 5 & 15 \\
$90-99 \%$ & 10 & 14 & 24 \\
$100 \%$ & 13 & 10 & 23 \\
total & 148 & 148 & 296 \\
\end{tabular}

TABLE 6-8. Results of the OPG-Gee (Gee) in 148 patients (296 sides) per side studied.

$\begin{array}{rrrrr}\text { degree of } & \text { OPG }(+) & \text { OPG }(-) & \text { OPG unknown } & \text { total } \\ \text { stenosis } & 16 & 109 & 11 & 136 \\ 0-9 \% & 3 & 15 & 2 & 20 \\ 10-19 \% & 2 & 17 & - & 19 \\ 20-29 \% & 2 & 3 & 1 & 6 \\ 30-39 \% & 3 & 16 & - & 19 \\ 40-49 \% & 1 & 5 & 1 & 7 \\ 50-59 \% & 7 & 7 & 1 & 15 \\ 60-69 \% & 3 & 8 & 1 & 12 \\ 70-79 \% & 10 & 3 & 2 & 15 \\ 80-89 \% & 17 & - & 3 & 24 \\ 90-99 \% & 17 & 187 & 6 & 23 \\ 100 \% & 81 & & 28 & 296 \\ 10 t a 1 & & & & \end{array}$

\section{6-3 Results of ocular pneumopletbysmography}

The result of the OPG-Gee was called positive if one of the criteria according to Gee, Eikelboom or Machleder as described in chapter 5 was met and was called negative if none of these criteria was met. The results, as obtained with the $\mathrm{OPG}$ Gee, will be described per side studied.

In 1,2 patients (24 sides) the OPG-Gee could not be performed because of contraindications (section 5-2). Furthermore, in 2 patients (4 sides) the results of 
the OPG-Gee could not be interpreted because of either inadvertent blinking of the eyes or hypentension. Therefore, the results of the OPG-Gee can be described for 268 arterial segments of the cerebropetal arteries. The results of the OPG-Gee examination are shown in table 6-8,6-9 and 6-10 for the interpretation according to Gee [OPG-Gee (Gee)], Eikelboom [OPG-Gee (Eikelboom)] and Machleder [OPG-Gee (Machleder)], respectiwely.

TABLE 6-9. Results of the OPG-Gee (Eikelboom) in 148 parients (296 sides) per side studied.

\begin{tabular}{|c|c|c|c|c|}
\hline $\begin{array}{l}\text { degree of } \\
\text { stenosis }\end{array}$ & $O P G(t)$ & OPG (-) & OPG unknown & total \\
\hline $0-9 \%$ & 4 & 121 & II & 136 \\
\hline $10-19 \%$ & - & 18 & 2 & 20 \\
\hline $20-29 \%$ & - & 19 & - & 19 \\
\hline $30-39 \%$ & - & 5 & 1 & 6 \\
\hline $40-49 \%$ & 2 & 17 & - & 19 \\
\hline $50-59 \%$ & - & 6 & 1 & 7 \\
\hline $60-69 \%$ & 5 & 9 & 1 & 15 \\
\hline $70-79 \%$ & 2 & 9 & 1 & 12 \\
\hline $80-89 \%$ & 8 & 5 & 2 & 15 \\
\hline $90-99 \%$ & 16 & 5 & 3 & 24 \\
\hline $100 \%$ & 17 & - & 6 & 23 \\
\hline total & 54 & 214 & 28 & 296 \\
\hline
\end{tabular}

TABLE 6-10. Results of the OPG-Gee (Machleder) in 148 parients (296 sides) per side studied.

\begin{tabular}{|c|c|c|c|c|}
\hline $\begin{array}{l}\text { degree of } \\
\text { stenosis }\end{array}$ & $O P G(+)$ & $\mathrm{OPG}(-)$ & OPG unknown & total \\
\hline $0-9 \%$ & 4 & 121 & 11 & 136 \\
\hline $10-19 \%$ & 1 & 17 & 2 & 20 \\
\hline $20-29 \%$ & - & 19 & - & 19 \\
\hline $30-39 \%$ & 1 & 4 & 1 & 6 \\
\hline $40-49 \%$ & 2 & 17 & - & 19 \\
\hline $50-59 \%$ & - & 6 & 1 & 7 \\
\hline $60-69 \%$ & 4 & 10 & 1 & 15 \\
\hline $70-79 \%$ & 1 & 10 & 1 & 12 \\
\hline $80-89 \%$ & 9 & 4 & 2 & 15 \\
\hline $90-99 \%$ & 17 & 4 & 3 & 24 \\
\hline $100 \%$ & 17 & - & 6 & 23 \\
\hline total & 56 & 212 & 28 & 296 \\
\hline
\end{tabular}


The sensitivity and the specificity of the OPG-Gee can be assessed if the results of the OPG-Gee are compared to the resules of arteriography. In table 6-11 the sensitivity and the specificity of the OPG-Gee examination are shown if the criteria for the interpretation of the OPG. Gee according to Gee, Eikelboom and Machleder are used.

TABLE 6-11. Sensitivity and specificity of the OPG-Gee examination to detect stenotic lesions in the cerebropetal arteries using criteria for the interpretation of the OPG-Gee according to Gee, Eikelboom and Machleder, per side studied.

\begin{tabular}{lrrrrrr} 
degree of & \multicolumn{2}{c}{ GEE } & \multicolumn{2}{c}{ EDKELBOOM } & \multicolumn{2}{c}{ MACFLEDER } \\
stenosis & sens & spec & sens & spec & sens & spec \\
$>=10 \%$ & 45 & 87 & 35 & 97 & 36 & 97 \\
$>=20 \%$ & 50 & 87 & 40 & 97 & 41 & 97 \\
$>=30 \%$ & 57 & 87 & 47 & 98 & 48 & 97 \\
$>=40 \%$ & 57 & 86 & 50 & 98 & 50 & 96 \\
$>=50 \%$ & 67 & 86 & 59 & 97 & 59 & 96 \\
$>=60 \%$ & 71 & 86 & 63 & 97 & 63 & 96 \\
$>=70 \%$ & 76 & 83 & 69 & 95 & 71 & 94 \\
$>=80 \%$ & 86 & 83 & 80 & 94 & 84 & 94 \\
$>=90 \%$ & 89 & 80 & 87 & 91 & 89 & 90 \\
$=100 \%$ & 100 & 75 & 100 & 85 & 100 & 84
\end{tabular}

The ability of the noninvasive test to detect stenotic lesions is greater for highgrade lesions than for low-grade lesions. Therefore, the sensitivity of the test increases if the degree of stenosis, used as a threshold to differentiate between 'healthy' and 'diseased', increases.

\section{6-3-1 Comments to the results of ocular pneumoplethysmography}

The most striking feature in the results of the OPG-Gee is the fact that the OPGGee could not be performed or interpreted in 12 cases with a stenosis of $70 \%$ or more. The OPG-Gee could nor be interpreted on 6 sides with a total occlusion of the ICA. The data in this study show a limited ability of the OPG-Gee to detect lesions of a minor or moderate degree of stenosis. Of the 22 sides with a stenosis between $50 \%$ and $70 \%$ dianseter reduction the OPG-Gee could be interpreted in 20 sides. The OPG-Gee detected such a lesion in 8,5 and 4 sides, if the examination was interpreted according to the results of Gee, Eikelboom and Machleder, respectively. Even high-grade stenotic lesions ( $>70 \%$ stenosis) are detected in a limited number of sides. The OPG-Gee could be interpreted in 62 of the 74 sides in which a high-grade stenosis was present. Of these 62 sides the OPG-Gee failed to detect stenotic lesions with a degree of narrowing of $70 \%$ or more if the criteria according to Gee, Eikelboom and Machleder are used in 15, 19 and 18 sides, 
respectively. Of the 222 arterial segments without a lesion or with a lesion less than $70 \%$ diameter reduction the OPG-Gee could be interpreted in 206 sides. The OPG-Gee showed a negative result in 172,195 and 194 sides, respectively.

The results of the OPG-Gee interpreted according to Eikelboom and Machleder are only slightly different. However, the criteria according to Gee for the interpretation of the OPG-Gee show a higher sensitivity, especially in the case of minor or moderate degrees of stenosis. These differences did not reach the level of statistical significance $(0.10>\mathrm{p}>0.05)$. The differences in the sensitivity and specificity of the OPG-Gee if different criteria for the interpretation of the test are applied in the present study corroborate the findings of Eikelboom (75). The data in this study suggest that a positive result of the OPG-Gee (Gee) has only a limited value for the detection of moderate or high-grade stenotic lesions in the cerebropetal arteries.

Comparison of the results of the OPG-Gee in this study with the results in other studies is hampered by the fact that the results of the OPG-Gee in the various studies are compared with different degrees of narrowing of the cerebropetal arteries, as seen at arteriography. Besides, in the present study the results of the OPG-Gee are described on a 'per side' basis. To facilitate the comparison of the results of the OPG-Gee examination in this study with those of other studies the sensitivity and the specificity of the OPG-Gee (Machleder) were also expressed on a "per patient' basis. The results of the OPG-Gee (Machleder) "per patient" compared to the results of arteriography on a "per patient" basis are shown in table 6-12.

TABLE 6-12. Results af the OPG-Gee, using the criteria according to Machleder, compared to the results of arteriography, per patient (148 parients).

\begin{tabular}{|c|c|c|c|c|}
\hline $\begin{array}{l}\text { degree of } \\
\text { stenosis }\end{array}$ & $O P G(t)$ & $\mathrm{OPG}(-)$ & OPG unknown & total \\
\hline $0-9 \%$ & 3 & 37 & 3 & 43 \\
\hline $10-19 \%$ & - & 7 & 1 & 8 \\
\hline $20-29 \%$ & - & 9 & - & 9 \\
\hline $30-39 \%$ & - & 3 & - & 3 \\
\hline $40-49 \%$ & - & 8 & - & 8 \\
\hline $50-59 \%$ & - & 4 & - & 4 \\
\hline $60-69 \%$ & 4 & 6 & 1 & 11 \\
\hline $70-79 \%$ & 2 & 5 & - & 7 \\
\hline $80-89 \%$ & 9 & 3 & 2 & 14 \\
\hline $90-99 \%$ & 14 & 3 & 3 & 20 \\
\hline $100 \%$ & 17 & - & 4 & 21 \\
\hline total & 49 & 85 & 14 & 148 \\
\hline
\end{tabular}


From the data in table 6-12 the sensitivity and specificity of the OPG-Gee (Machleder) per patient studied are derived. These values are shown in table 6-13.

TABLE 6-13. Sensitivity and specificity of the OPG-Gee (Machleder) per patient studied (148 parients).

$\begin{array}{lcc}\text { degree of } & \text { OPG }- \text { Gee } & (\text { Mach leder } \\ \text { stenosis } & \text { sensitivity } & \text { specificity } \\ >=10 \% & 49 & 93 \\ >=20 \% & 53 & 94 \\ >=30 \% & 59 & 95 \\ >=40 \% & 61 & 95 \\ >=50 \% & 69 & 96 \\ >=60 \% & 73 & 96 \\ >=70 \% & 79 & 91 \\ >=80 \% & 87 & 90 \\ >=90 \% & 91 & 82 \\ >=100 \% & 100 & 73\end{array}$

If the OPG-Gee was used on a 'per parient' basis rather than on a 'per side' basis of the 62 cases with stenotic lesions with a diameter reduction of $70 \%$ or more 53 cases could be interpreted. Of these 53 cases a positive $O P G-G$ in 42 cases, and a negative OPG-Gee in 11 cases. Of the 86 cases without a stenosis or with a stenosis of less than $70 \%$ diameter reduction the OPG-Gee could be interpreted in 81 cases. The OPG-Gee showed a negative result in 74 of these cases, and a false positive result in 7 of these cases.

In the present study the sensitivity of the OPG-Gee, if used on a 'per patient' basis, is increased compared to the sensitivity of the OPG-Gee used on a 'per side' basis. The specificity of the OPG-Gee if used on a 'per patient' basis is diminished as compared to the the specificity of the test if used on a 'per side' basis. The differences in specilicity of the test are less pronounced than the differences in sensitivity. The differences in sensitivity and specificity were not statistically significant for lesions of $70 \%$ diameter reduction or more $(0.5>p>0.1)$.

Gee repeatedly $(90,91,92,93,94,184)$ modified his criteria for the interpretation of the OPG-Gee after retrospective analysis of the results and after changes in the OPG-Gee instrument. Gee's criteria for the interpretation as described in this study are derived from a study in 1982 (94). In this study on over 600 patients the result of arteriography was considered "positive" if at the origin of the ICA a lesion was seen of $50 \%$ diameter reduction of the carotid bulb or more. The sensitivity and specificity of the test, on a "per side" basis, was $92 \%$ and $96 \%$ 
respectively. These results are better than the results obtained in this present study.

Eikelboom (72) describes the sensitivity and specificity of the OPG-Gee examination for a range of diameter reductions, from $50 \%$ to $99 \%$. The OPG-Gee was used on a 'per patient' basis, using his own criteria. The sensitivity and specificity of the OPG-Gee for the detection of lesions of $50 \%$ stenosis or more was $74 \%$ and $85 \%$, respectively, compared to a sensitivity and a specificity of the OPG-Gee (Machleder) on a 'per patient' basis of $69 \%$ and $96 \%$, respectively, in the present study. Eikelboom also compared the criteria according to Gee, Eikelboom and Machleder for their ability to detect arteriographic lesions with a diameter reduction of $65 \%$ or more. The sensitivity was $84 \%, 87 \%$ and $83 \%$, respectively and the specificity $80 \%, 84 \%$ and $84 \%$, respecrively. This comparison was made on a 'per parient' basis. A fair comparison of these results to those obtained in the present study is hampered because of the fact that a diameter reduction of $65 \%$ was not used as a threshold in the present study, and the 'per patient'basis of Eikelboom"s study. However, for the OPG-Gee (Machleder) the results can be compared to those shown in table 6-13. By interpolation it can be estimated that the sensitivity of the OPG-Gee (Machleder) performed by Eikelboom is higher, whereas the specificity is lower in comparison to the results of the present study.

Machleder and collaborators $(10,11)$ described the results of the OPG-Gee on a "per parient' basis, for lesions with an arteriographically determined degree of stenosis of $65 \%$ or more. The sensitivity and specificity of the rest was $87 \%$ and $96 \%$, respectively. These results compare favorably to the results obtained in the present study (table 6-13) and seem far better than those reported by Gross et al (101). Similar results were reported by other investigators $(75,171,172,186)$, although different criteria were used for the interpretation of the noninvasive test and arteriography.

The limited sensitivity of the OPG-Gee in detecting lesions with a lesser degree or even a severe degree of stenosis may reduce the applicability of this examination as a solitary test in the detection of obstructions in the cerebropetal arteries. The most important advantage of the OPG-Gee over other noninvasive techniques is its ability to estimate the contribution of the collateral circulation because the OAP as measured is the resultant of the pressure transferred both across the stenosis and the collateral vessels $(71,94,179,210)$.

6-4 Results of the periorbital Doppler examination

The result of the periorbital Doppler examination (OSM) was called positive if one of the criteria as mentioned in chapter 5 was met and the result was called negative if none of these criteria was met. The results will be described per side 
studied. The results of the OSM will be compared to the results of arteriography, per side, in the arterial segments between the aortic arch and the ophthalmic artery as shown in table 6-7. The OSM could not be interpreted on both sides in 1 parient because of lack of cooperation in the performance of the test. The results of the OSM examination are shown in table 6-14.

TABLE 6-14. Results of the OSM examination in 148 patients (296 sides).

\begin{tabular}{lcccc}
\hline $\begin{array}{c}\text { degree of } \\
\text { stenosis }\end{array}$ & OSM $(+)$ & OSM $(-)$ & OSM unknown & total \\
$0-9 \%$ & 5 & 130 & 1 & 136 \\
$10-19 \%$ & 1 & 19 & - & 20 \\
$20-29 \%$ & 1 & 17 & 1 & 19 \\
$30-39 \%$ & - & 6 & - & 6 \\
$40-49 \%$ & 2 & 17 & - & 19 \\
$50-59 \%$ & 1 & 6 & - & 7 \\
$60-69 \%$ & 7 & 8 & - & 15 \\
$70-79 \%$ & 3 & 9 & - & 12 \\
$80-89 \%$ & 7 & 8 & - & 15 \\
$90-99 \%$ & 18 & 6 & - & 24 \\
$100 \%$ & 23 & - & - & 23 \\
tonal & 68 & 226 & 2 & 296 \\
& & & &
\end{tabular}

A positive OSM was found on 51 of the 74 sides with a degree of narrowing of $70 \%$ or more of the arterial segment between the aornic arch and the ophthalmic artery. The OSM was negative on 23 sides in this group. Of the 222 sides without a stenosis or a with a stenosis of less than $70 \%$ a positive OSM was found on 17 sides, and a negative OSM 203 sides. The sensitivity and specificity of the OSM were calculated for increasing degrees of stenosis in steps of 10\%. Therefore, a comparison between the results of the OSM and the OPG-Gee in this study can be made. The results of the OSM, expressed as sensitivity and specificity, are shown in table $6-15$.

\section{6-4-1 Comments to the results of the periorbital Doppler examination}

The results of the OSM in this study can be hardly compared with those obtained in other investigations because the criteria for the interpretation vary widely from study to study. Only the absence or reversal of the direction of periorbital flow is generally acknowledged as one of the criteria for an abnormal result of the OSM $(16,36,65,80,138,201)$. Furthermore, the OSM can be performed with or without compression of the CCA. The sensitivity of the OSM in the various reports ranges from $59 \%(101,267)$ to $98.7 \%$ (16) for the detection of "significant stenotic lesions" of the ICA.

In the interpretation of the OSM examination attempts liave been made to relate characteristics of the sonogram of the supraorbital arteries to the patency of the 
TABLE 6-15. Sensitivity and specificity of the OSM described for increasing degrees of stenosis in steps of $10 \%$.

$\begin{array}{lcc}\text { degree of } & \text { sensitivity } & \text { specificity } \\ \text { stenosis } & 40 & 96 \\ >=10 \% & 45 & 96 \\ >=20 \% & 50 & 96 \\ >=30 \% & 53 & 96 \\ >=40 \% & 61 & 95 \\ >=50 \% & 65 & 95 \\ >=60 \% & 69 & 92 \\ >=70 \% & 77 & 91 \\ >=80 \% & 87 & 89 \\ >=90 \% & 100 & 83 \\ >=100 \% & & \end{array}$

ICA $(23,211)$. The presence of a 'presystolic notchn' as described by Kadota et al (129) was reported after the start of this study. Therefore, their $89 \%$ accuracy in the differentiation between severe stenosis and total occlusion of the ICA reached in a (retrospective ?) study of 44 patients could not be evaluated in this study. The ratio of the first and second systolic peak (A/B ratio), as described by Gosling et al $(23,211)$, reached in their hands a sensitivity and a specificity for the detection of stenotic lesions in the cerebropetal arteries of $89 \%$ and $92 \%$, respectively (166). The use of the $\mathrm{A} / \mathrm{B}$ ratio of the periorbital arteries before and after compression of the branches of the ECA proved to be of no value for the discrimination of 'normal' and 'diseased" ICAs in the present study (figure 6-2 and figure 6-3).

\section{6-5 Comparison of the results of the indirect tests}

The most striking difference between the OPG-Gee and the OSM is the number of failures in the former test. The OPG-Gee could not be pertormed or interpreted on 28 sides and the OSM could not be performed on 2 other sides. Of the 74 sides of a stenotic lesion with a diameter reduction of $70 \%$ or more a positive result of an indirect test was acquired on $47,43,44$, and 51 sides for the OPG-Gee (Gee), the OPG-Gee (Eikelboom), the OPG-Gee (Machleder) and the OSM respectively, and a false negative result on $15,19,18$, and 23 sides, respectively. The OSM showed a positive result on all 23 sides with an occlusion of the ICA, whereas the OPG-Gee could not be performed in all patients and showed a positive result on only 17 sides. These differences in the sensitivity and specificity between the interpretations of the OPG-Gee according to Eikelboom and Machleder and the OSM are small. The OSM barely fails and there are no real contraindications for this test. Therefore, the OSM may be preferred ower the OPG-Gee, as far as the detection of stenotic lesions is concerned. For the quantification of the overall collateral circulation the OPG-Gee may be preferred, as it also takes the collateral circulacion through the circulus arteriosus into consideration. 


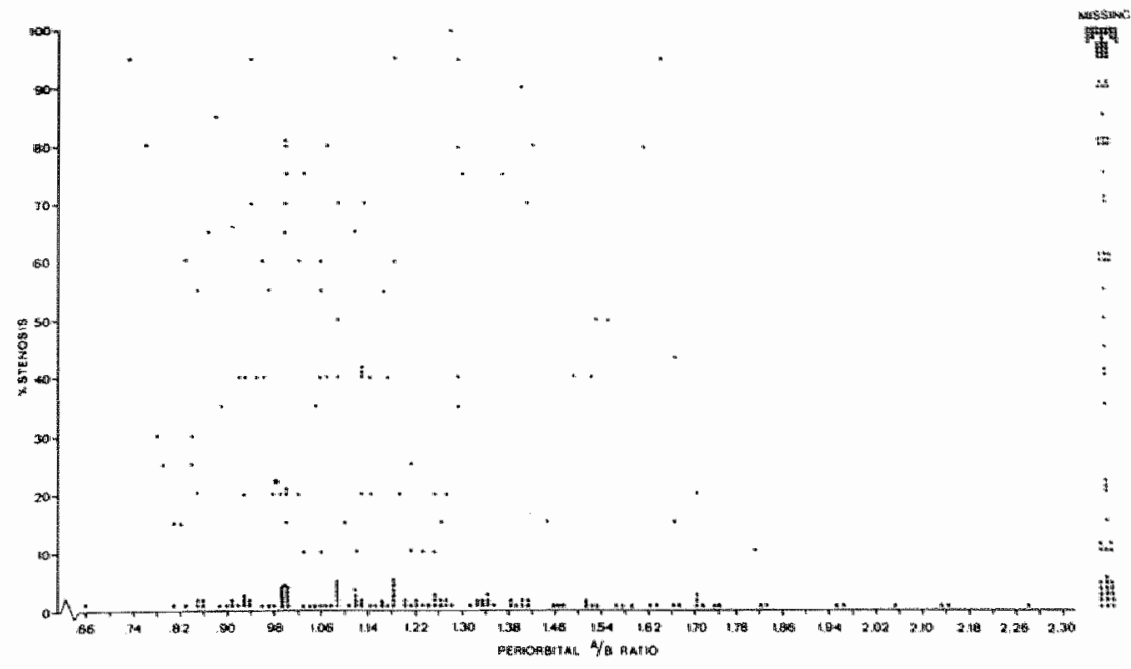

Figure 6-2. Diagram showing the relation between the degree of stenosis in the arterial segment from the aortic arch to the ophthalmic artery (on the vertical axis) and the $A / B$ ratio, i.e. the ratio of the first and second systolic peak of the sonograms of the periorbial Doppler examination, as described by Gosling et al. The diagram is discussed in section 6-4-1.

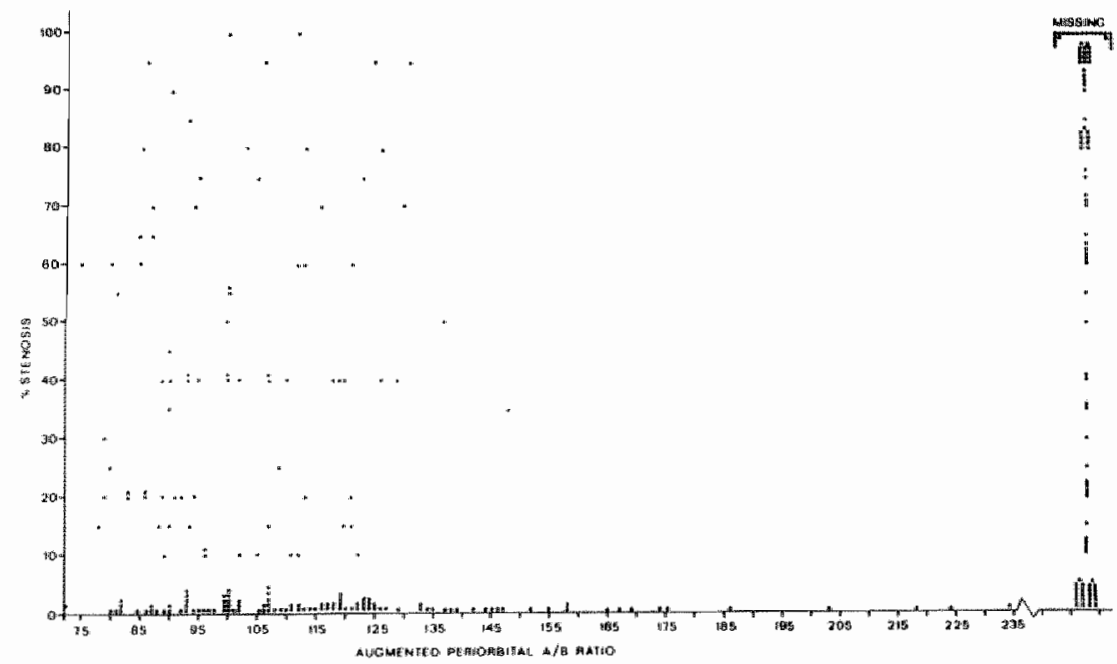

Figure 6-3. Diagram showing the relation between the degree of stenosis in the arterial segment from the aortic arch to the ophthalmic artery (on the vertical axis) and the augmented $A / B$ ratio, i.e. the ratio of the first and second systolic pak of the sonograms of the periorbital Doppler examination, as described by Gosling et al, after compression of the branches ofthe externall carotid artery. The diagram is discussed in section $6-4-1$. 
The results of the OPG-Gee were combined with the results of the OSM for the various degrees of stenosis. For this combination the $O P G$-Gee was, arbitrarely, interpreted with the criteria according to Machleder. The results are shown in Table 6-16. The results of the OPG-Gee (Machleder) and the OSM could be combined for 266 sides. Of the 62 sides in which a lesion with a degree of narrowing of $70 \%$ or more was found at arteriography both the OPG-Gee and the OSM were positive on 35 sides, while both examinations were negative on 13 sides. The results of the indirect examinations were contradictory on 14 sides. On 9 out of these 14 sides the OPG-Gee was indicarive of a severe stenosis while the OSM showed a negative result. A direct relation to the presence of a stenosis in the ECA in these cases could not be found, as the ECA only showed a stenosis on 4 of the 14 sides. The OSM showed a positive result on 1 side while the OPG-Gee showed a negative result and the origin of the ECA presented a $80 \%$ stenosis. Once the OSM showed a negative result, and the OPG-Gee a positive result, but the lesion at the origin of the ECA was estimated as $40 \%$ diancter reduction. In only 2 of the 14 sides in which the results of the OSM and the OPG-Gee were contradictory the negative result of the OSM might be caused by a severe stenosis in the origin of the ECA ( $80 \%$ and $95 \%$ diameter reduction, respectively). Of the 204 cases without a lesion or with a lesion of less than $70 \%$ narrowing both indirect examinations were negative in 185 cases, while the examinations were positive in 9 cases. In this category the results of the OPG-Gee and the OSM were contradictory in 10 cases. In 7 of these cases the OSM showed a positive result.

TABLE 6-16. Combination of the results of the OPG-Gee (Machleder) and the OSM in 148 patients (296 sides).

\begin{tabular}{|c|c|c|c|c|c|c|c|c|c|c|}
\hline \multirow{3}{*}{$\begin{array}{l}\text { degree of } \\
\text { stenosis }\end{array}$} & \multicolumn{3}{|c|}{ OPG (t) } & \multicolumn{3}{|c|}{$\mathrm{OPG}(-)$} & \multicolumn{3}{|c|}{$O P G(?)$} & \multirow{3}{*}{ rotal } \\
\hline & OSM & OSM & OSM & OSM & OSM & OSM & OSM & OSM & OSM & \\
\hline & + & - & $?$ & 4 & - & $?$ & + & - & $?$ & \\
\hline $0-9 \%$ & 3 & 1 & - & 2 & 118 & 1 & - & $1 \|$ & - & 136 \\
\hline $10-19 \%$ & $-m$ & 1 & - & 1 & 16 & - & - & 2 & - & 20 \\
\hline $20-29^{\circ} \%$ & - & - & - & 1 & 17 & 1 & - & - & - & 19 \\
\hline $30-39 \%$ & - & 1 & - & - & 4 & - & - & 1 & - & 6 \\
\hline $40-49 \%$ & 2 & - & - & - & 17 & - & - & - & - & 19 \\
\hline $50-59 \%$ & - & - & - & 1 & 5 & - & - & $\mathbb{1}$ & - & 7 \\
\hline $60-69 \%$ & 4 & - & - & 2 & 8 & - & 1 & $\ldots$ & - & 15 \\
\hline $70-79^{\mathrm{th} / \mathrm{s}}$ & - & 1 & - & 2 & 8 & - & 1 & - & - & 12 \\
\hline $80-89 \%$ & 4 & 5 & - & 1 & 3 & - & 2 & - & - & 15 \\
\hline $90-99^{10 / 4}$ & 14 & 3 & - & 2 & 2 & - & 2 & 1 & - & 24 \\
\hline $100 \%$ & 17 & - & 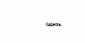 & - & - & - & 6 & - & - & 23 \\
\hline total & 44 & 12 & - & 12 & 198 & 2 & 12 & 16 & - & 296 \\
\hline
\end{tabular}


During the direct Doppler examination a decision was made regarding the degree of spectral broadening of the Doppler signal as observed in the CCA, the ECA and the $\mathbb{C} \mathrm{CA}$. The degree of spectral broadening was classified as "Grade 1 " through "Grade 5" according to the criteria described in chapter 5 . The results of the direct Doppler examination describing the degree of spectral broadening of the Doppler signal are compared with the degree of stenosis found at arteriography in increasing steps of narrowing of $10 \%$. The relarion between the degree of spectral broadening and the results of arteriography of the ICA is shown in table 6-17.

TABLE 6-17. Relation between the degree of stenosis of the ICA as observed at arteriography and the degree of spectral broadening of the direct Doppler examination in 148 patients (296 sides).

degree of

stenosis

0. $9 \%$

$10-19 \%$

$20-29 \%$

$30-39 \%$

$40-49 \%$

$50-59 \%$

$60-69 \%$

$70.79 \%$

$80-89 \%$

$90-99 \%$

$100 \%$

total

\begin{tabular}{rcr} 
& \multicolumn{3}{c}{ Grade 1} & Grade 2 & Grad \\
134 & 6 & - \\
18 & 2 & - \\
16 & 4 & - \\
1 & 5 & - \\
1 & 17 & 2 \\
- & - & 6 \\
- & - & 12 \\
- & 1 & 5 \\
- & - & 5 \\
- & 1 & 2 \\
- & - & - \\
170 & 36 & 32
\end{tabular}

spectral broadening -...

de 3 Grade 4 Grade 5 rotal

$\begin{array}{rrrr}- & - & 1 & 141 \\ - & - & - & 20 \\ - & - & - & 20 \\ - & - & - & 6 \\ 2 & - & - & 20 \\ 6 & 1 & - & 7 \\ 2 & 2 & - & 14 \\ 5 & 3 & - & 9 \\ 5 & 8 & - & 13 \\ 2 & 21 & - & 24 \\ - & 2 & 20 & 22 \\ 2 & 37 & 21 & 296\end{array}$

In 155 ICAs a stenosis of $10 \%$ or more was found an arteriography. In 119 of these cases a Doppler signal with spectral broadening or an absent Doppler signal was found. On the 119 sides with a Doppler signal showing spectral broadening or absence of the Doppler signal a "Grade 2" pattern was found on 30 sides, a "Grade 3" pattern on 32 sides, a "Grade 4" pattern on 37 sides and absence of the Doppler signal on 20 sides. Of the $14 \|$ ICAs without a stenosis or with a stenosis of less than $10 \%$ diameter reduction a "normal' Doppler signal, showing no spectral broadening, was found on 134 sides. In 6 of the 141 sides a "Grade 2 " pattern was found, showing minimal spectral broadening. In 1 of the 141 sides no Doppler signal of the ICA could be obtained, thus arousing the talse impression of an occluded ICA. On the other hand, in 36 of the 170 Doppler examinations of the ICA showing a 'normal' Doppler signal, a stenosis at the origin of the ICA 
was found. These false negative Doppler examinations were obtained from 18 ICAs with a stenosis of $10 \%$ to $19 \%$, from 16 ICAs with a stenosis of $20 \%$ to $29 \%$, from 1 ICA with a stenosis of $30 \%$ to $39 \%$ and 1 ICA with a stenosis berween $40 \%$ and $49 \%$. It should be mentioned that on 2 sides a severe degree of spectral broadening was found ("Grade 4" pattern), while the ICA was found to be occluded at arteriography. However, it cannot be excluded that these very tight lesions have been missed at arteriography, assuming that the Doppler signal originated from the ICA. On 5 sides a severe stenotic lesion was present proximal to the carotid artery bifurcation (Table 6-3). In these cases the interpretation of the sonogram of the ICA and the ECA was performed reluctantly. Furthermore, on another 9 sides the estimation of the degree of spectral broadening was hampered by the presence of a short, fat or rigid neck. Nevertheless, all examinations were concluded by the allocation of the degree of spectral broadening to one of the live categories of spectral broadening previously described. A graphic representation of the degree of spectral broadening and the degree of stenosis at the origin of the ICA is shown in figure 6-4.

Spectral broadening of the Doppler signal obtained from ICAs without stenotic lesions can be caused by irregularities in the CCA or by elongation of the ICA. Therefore, the presence of mild spectral broadening in the Doppler signal of the ICA does not automatically imply the presence of a stenosis in the ICA. These fearures will be further discussed in chapter 7 . If merely the presence of spectral broadening in the Doppler signal of the ICA is considered indicative of a stenosis in this artery the sensitivity and specificity of the direct Doppler examination can be calculated from the data in table 6-17. The values for these parameters are shown in table 6-18.

TABLE 6-18. Sensirivity and specificity of the direct Doppler examination of the ICA carotid artery and the peak systolic frequency shift of the Doppler signal of the internal

\begin{tabular}{lcc} 
degree of & \multicolumn{2}{c}{ direct Doppler examination } \\
stenosis & sensitivity & specificity \\
$>=10 \%$ & 77 & 95 \\
$>=20 \%$ & 87 & 94 \\
$>=30 \%$ & 98 & 93 \\
$>=40 \%$ & 99 & 90 \\
$>=50 \%$ & 100 & 82 \\
$>=60 \%$ & 100 & 79 \\
$>=70 \%$ & 100 & 75 \\
$>=80 \%$ & 100 & 72 \\
$>=90 \%$ & 100 & 68 \\
$>=100 \%$ & 100 & 62
\end{tabular}

In the direct Doppler examination of the ICA the maximal peak systolic frequency shift of the Doppler signal was recorded. At the site of a stenosis the peak 


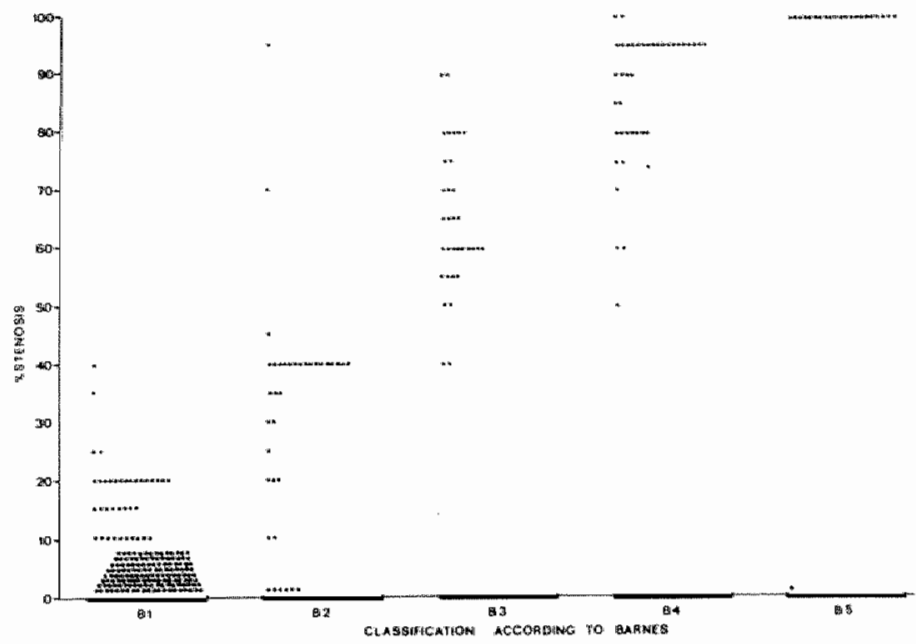

Figare 6-4. Diagram showing the relation berween the degree of stenosis in the internal carotid artery and the degree of spectral broadening, classified according to Barnes, in the Doppler signal of the internal caratid artery in 148 patients (296 sides). "The degree of stenosis is shown on the vertical axis and the five degrees of spectral broadening, indicared as B1 to B5 for Grade 1 to Grade 5 respectively, are shown on the horizontal axis.

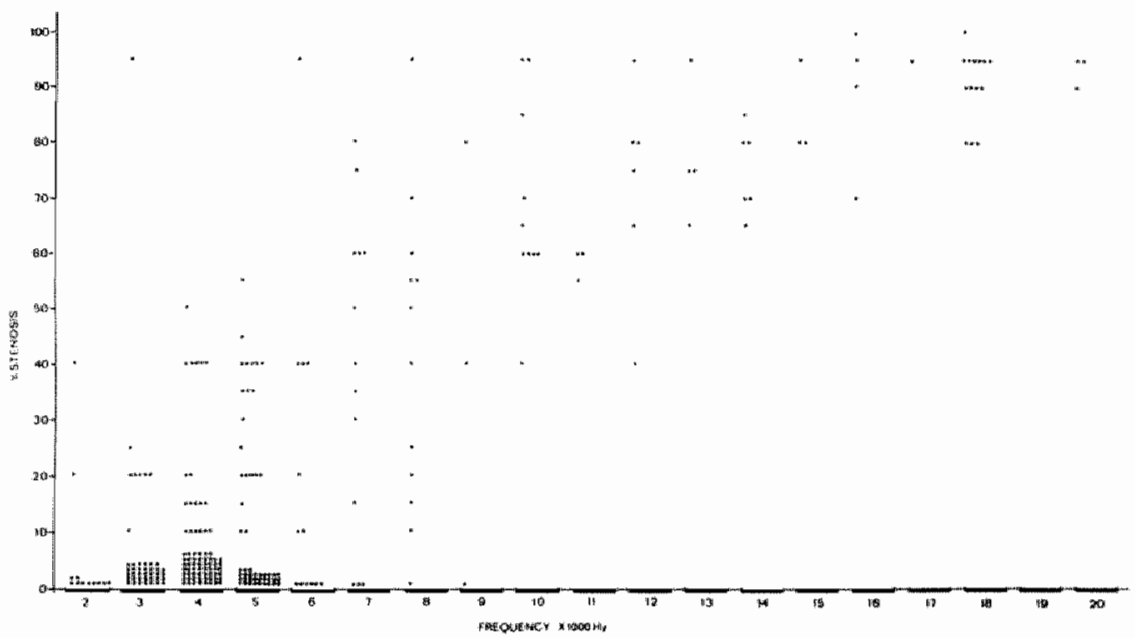

Figure 6-5. Diagram showing the relation between the degree of stenosis in the internal carorid artery and the peak systolic frequency shift of the Doppler signal of the internal carotid arrery in 148 patients (296 sides). The degree of stenosis is shown on the vertical axis and the peak systolic frequency shift is shown on the horizontal axis. 
systolic frequency increases in proportion to the severity of the stenosis $(46,154)$. The increases in the peak systolic frequencies with the transition from a minor to a moderate stenosis are small compared to the potential changes that can occur by altering the angle of insonation (64). In the present study this peak systolic frequency shift was obtained without an imaging technique of the carotid arteries. Therefore, the assessment of the peak systolic frequency shifts in the CCA and the $\mathrm{JA}$ cannot be compared with those described by Blackshear et al (31). The relation between the peak systolic frequency shift of the Doppler signal and the degree of stenosis of the ICA is shown in figure 6-5. It should be noted that these frequency shifts are obtained with a Doppler probe with an $8 \mathrm{MHz}$ emission frequency, or corrected for if they were obtained with a $5 \mathrm{MHz}$ Doppler probe. The data in figure 6-5 indicate that a peak systolic frequency of $9 \mathrm{kHz}$ or more is always associated with moderate or severe stenosis of the ICA. On the other hand, even severe stenotic lesions can be present if the peak systolic frequency shift does not exceed 2 to $3 \mathrm{kHz}$. These findings are in agreement with the observations of Spencer (251), indicating that the peak systolic blood flow velocity decreases if the degree of stenosis exceeds a diameter reduction of approximately $90 \%$. Whether by combining the degree of spectral broadening and the peak systollic frequency shift more accurate information can be obtained about the severeness of the stenosis will be discussed in section 6-6-1.

6-6-1 Comments to the results of the direct Doppler examination of the internal carotid artery

The data in table 6-17 clearly demonstrate the ability of the direct Doppler examination using spectral frequency analysis of the Doppler signal to detect the presence of stenotic lesions in the cervical carotid artery. Even if only spectral broadening of the Doppler signal is used as a criterion, the results of the direct Doppler examination of the ICA are superior to the results of the indirect methods of examination. Spectral broadening of the Doppler signal occurs in the presence of mild, moderate and severe stenotic lesions. The degree of spectral broadening of the Doppler signal is used as a criterion the results of the direct also shown by Barnes et al (15), Brown et al (46) and Douville et al (64). The "Grade 2" pattern of spectral broadening seems to be a transitional partern. This pattern can be observed in the Doppler signal obtained from arteries without stenotic lesions and from arteries with minor degrees of stenosis. Spectral frequency analysis of the Doppler signal also allows the measurement of the peak systolic frequency shift at the site of the lesion. The peak systolic frequency shift increases with the degree of stenosis, up to very high grade stenotic lesions $(90 \%$ diameter reduction). If the peak systolic frequency shift of the Doppler signal is considered together with the degree of spectral broadening of this Doppler signal (tigure 6-6) a more accurate estimation of the degree of stenosis is possible than with determining peak frequency alone. For example, in figure 6-6 the result of the direct Doppler examination showing a $4 \mathrm{kHz}$ peak systolic frequency shift 


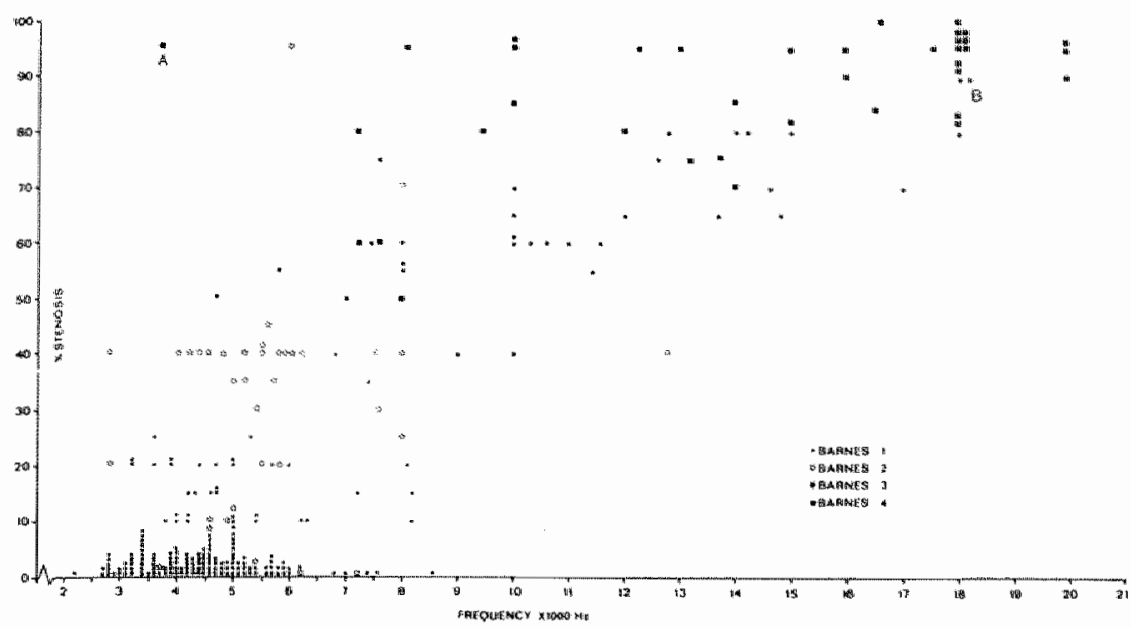

Figure 6-6. Diagram showing the relation between the degree of stenosis in the incernal carovid artery (on the vertical axis), the peak systolic frequency shift of the Doppler signal of the internal carotid artery (on the horizontal axis) and the degrec of spectral broatening of the Doppler signal of the internal carotid artery (shown as different markers for the various frequency shifts) in 148 parients (296 sides).

with a stenosis of $95 \%$ diameter reduction (indicated as - A-) will be recognized as a severe stenoric lesion because the degree of spectral broadening ("Grade 4") is taken into consideration. This lesion would have been missed when considering peak frequency alone. It may be expected that the increase in the degree of spectral broadening and the increase in the peak systolic frequency shift of the Doppler signal are related. In this study the, subjecrive, estimation of the degree of spectral broadening, as 'Grade 1' to 'Grade 5' and the peak systolic frequency shift, in $\mathrm{kHz}$, were multiplied to explore whether the new parameter would lead to a more accurate diagnosis of the lesions in the ICA. For example, the lesion in tigure 6-6 indicated as -A-presents a "Grade 4 " degree of spectral broadening with a peak systolic frequency shift of $4 \mathrm{kHz}$. If multiplied the effect will be 16 (a dimensionless number). The results are shown in ligure 6-7. The peak systolic frequency shift cannot be measured in the case of an occluded artery. Furthermore, the determination of the peak systolic frequency is difficult, or impossible, if the Doppler spectrum lacks a distinct upper band width. Therefore, the results in figure 6-6 and 6-7 will be shown for less than 296 samples.

The direct Doppler examination can be interpreted according to the degree of spectral broadening, the peak systolic frequency shift, or the combination of both. The sensitivity and the specificity of the direct Doppler examination for the various degrees of stenosis, if only the degree of spectral broadening was used as a criterion, are shown in table 6-18. To compare the results of the direct Doppler 


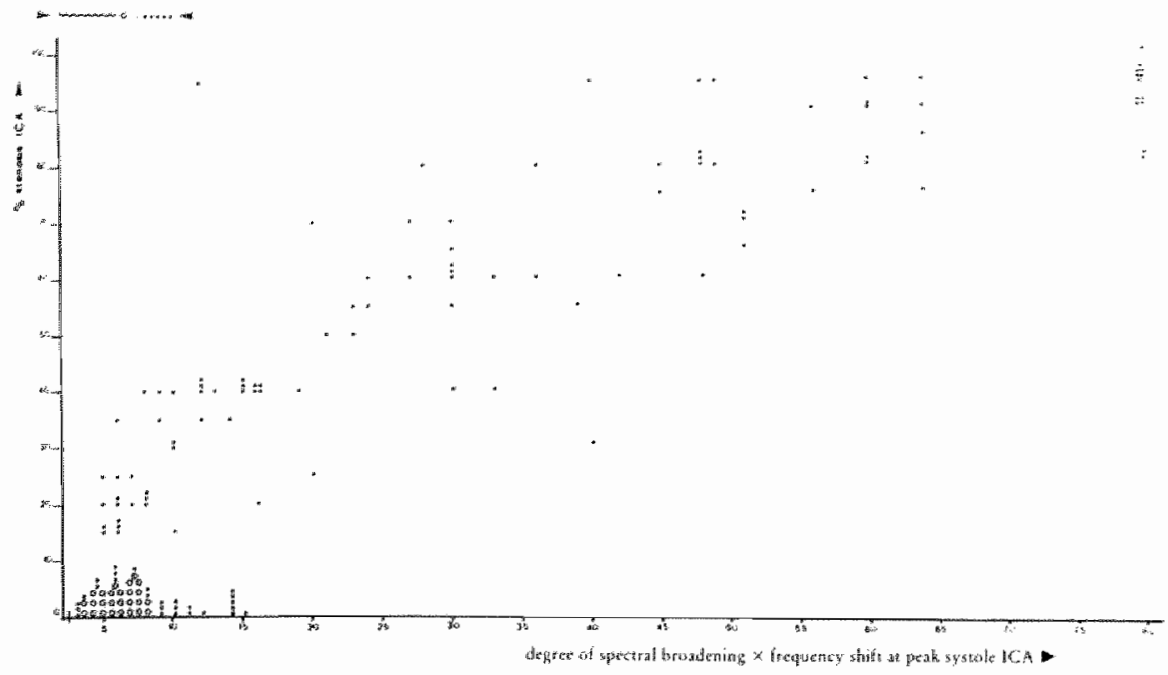

Figure 6-7. In this diagram the degree of spectral broadening is mutriplied by the peak systolic frequency shift of the Doppler signal, as described in section 6-6 1. This multiplication results in a dimensionless number. "The degree of stenosis in the internal carotid artery is shown on the wertical axis, and the outcome of the "mulciplication' is shown on the horizontal axis.

examination if the degree of spectral broadening, the peak systolic frequency, or the multiplication of both are used as a criterion $\mathrm{ROC}$-curves were prepared. These curwes are presented for the degree of stenosis of the ICA of $30 \%$ or more. The ROC-curves are presented in figure 6-8. The threshold for the interpretation of the degree of spectral broadening is set at the 'Grade 2' and the 'Grade 3' level. A lower degree of spectral broadening is inappropriate, and higher degrees were not used because then the differentiation between normal and abnormal is lost. 'The sensitivity and the specificity of the peak systolic frequency shift and the "muttiplication" are shown for a wider range of thresholds. The thresholds are indicated in figure 6-8. It is obvious that the subjective interpretation of the degree of spectral broadening is superior to the assessment of the peak systolic frequency shift, or the "multiplication".

Especially the sensitivity of the direct Doppler examination is superior to the sensitivity of the indirect tests. The specificity of the direct Doppler examination is less than that obtained with the indirect tests. This is caused by the increasing number of so-called "false positive" direct Doppler examinations. The results of the direct Doppler examination in the present study compare faworably with the results of Bandyk et al $(14)$, Barnes et al $(18,19)$, Arbeille et al (4) and Brown et al (48), all using spectral broadening of the CW Doppler signal. The results of this 


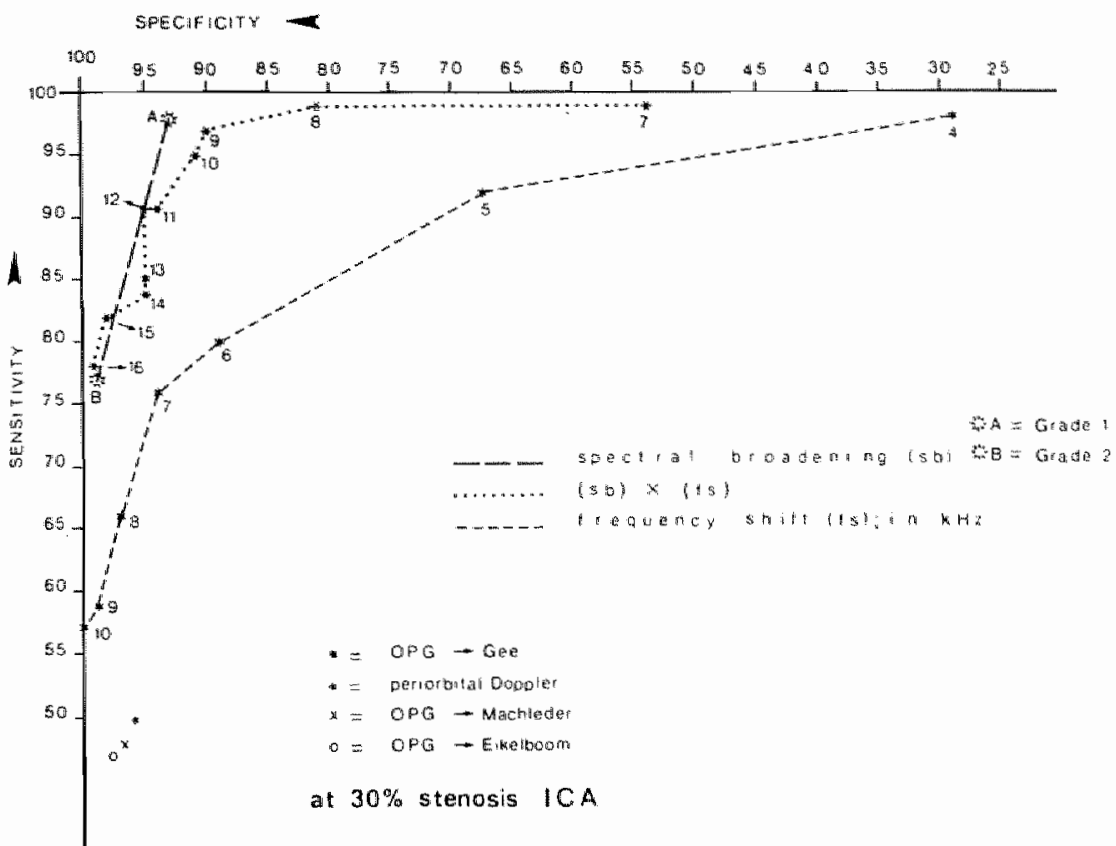

Figure 6 8. ROC-curve showing the rellation between the sensitivicy and the specificity of the various interpretations of the direct Dopplen examinations for the detecrion of stenotic lesions with $30 \%$ diameter reduction or more in the internal carorid artery. If the degrec of spectral brondening is used as a criterion only two thresholds are considered: "Grade 2" spectral broadening indicated as A and "Grade 3" spectral broadening indicated as B. If the peak systolic frequency shift is used as a criterion the thresholds are: $4=4 k \mathrm{~Hz}, 5=5$ $\mathrm{kHz}$, and so on. If the multiplication of the degree of spectral broadening and the peak systolic frequency shift is used as a criterion the thresholds indicate the resulting number. The values of the sensitivity and the specificity of the indirect methods of examination for the detection of stenoses of $30 \%$ or more are also plotred. From this ROC-curve it is obvious that the estimation of the degree of spectral broadening is superior to the peak trequency analysis and the indirecr techniques in the derection of stenotic lesions.

study corroborate our earlier findings on spectral analysis of the CW Doppler signal of the ICA $(8,9)$.

The direct Doppler examination of the cervical carotid arteries can be expected to differentiate between sewerestenosis and total occlusion of the ICA. Therefore, in three cases the results of the direct Doppler examination merit further explanation. In one case no Doppler signal could be obtained from the ICA, while the Doppler signal of the CCA was compatible with an occlusion of the ICA. In this patient both indirect tests were indicative of a hemodynamically signiticant stenosis on the same side. At arteriograpty, approwimately 48 hours later, no lesion could be detected at the ICA. The repeated noninvasive tests (not included 


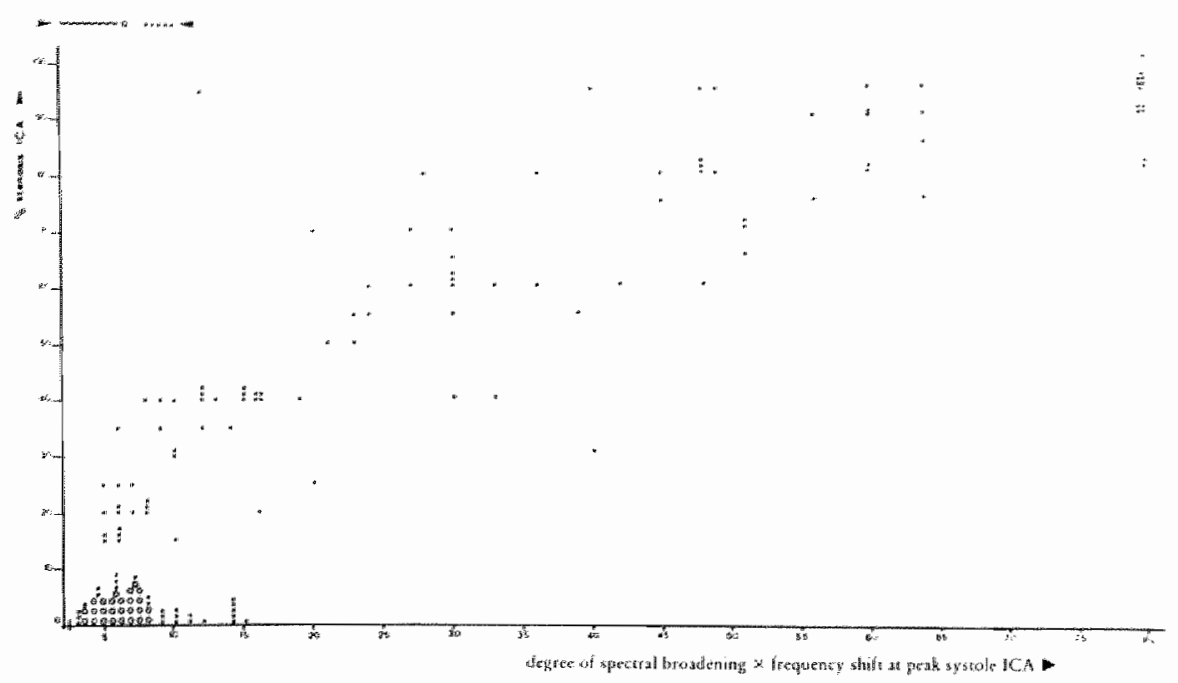

Figure 6-7. In this diagram the degree of spectral broadening is multiplied by the peak systolic frequency shife of the Doppler signal, as described in section 6-6-1. This multiplication results in a dimensionless number. The degree of stenosis in the internal carotid artery is shown on the vertical axis, and the outcome of the "multiplication" is shown on the horizonal axis.

examination if the degree of spectral broadening, the peak systolic frequency, or the multiplication of both are used as a criterion ROC-curves were prepared. These curves are presented for the degree of stenosis of the ICA of $30 \%$ or more. The ROC-curves are presented in figure 6-8. The threshold for the interpretation of the degree of spectral broadening is set at the 'Grade 2' and the 'Grade 3' level. A lower degree of spectral broadening is inappropriate, and higher degrees were not used because then the differentiation between normal and abnormal is lost. The sensitivity and the specificity of the peak systolic frequency shift and the "multiplication" are shown for a wider range of thresholds. The thresholds are indicated in figure 6-8. It is obvious that the subjective interpretation of the degree of spectral broadening is superior to the assessment of the peak systolic frequency shift, or the "multiplication".

Especially the sensitivity of the direct Doppler examination is superior to the sensitivity of the indirect tests. The specificity of the direct Doppler examination is less than that abtained with the indirect tests. This is caused by the increasing number of so"called "false positive" direct Doppler examinations. The results of the direct Doppler examination in the present study compare favorably with the results of Bandyk et al (14), Barnes et al $(18,19)$, Arbeille et al (4) and Brown et al (48), all using spectral broadening of the CW Doppler signal. The results of this 


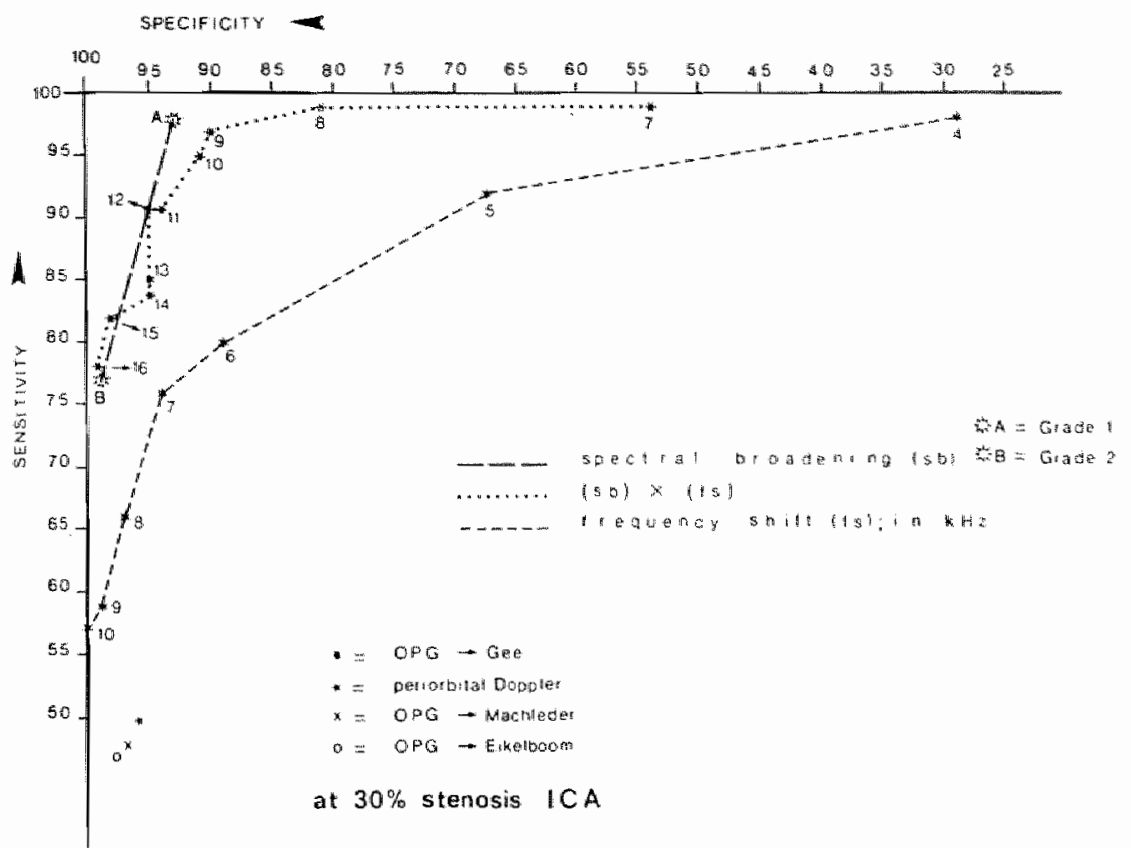

Figure 6-8. ROC-curve showing the relation between the sensitivity and the specificity of the various interpretations of the direct Doppler examinations for the detection of stenotic lesions with $30 \%$ diameter reduction or more in the internal carotid artery. If the degrec of spectral broadening is used as a criterion only two thresholds are considered: "Grade 2" spectral broadening indicated as A and "Grade 3" spectral broadening indicaned as B. If the peak systolic frequency shift is used as a criterion the thresholds are: $4=4 \mathrm{kHz}, 5=5$ $\mathrm{kHz}$, and so on. If the multiplication of the degree of spectral broadening and the peak systolic frequency shift is used as a criterion the thresholds indicate the resulting number. The values of the sensitivity and the specificity of the indirect methods of examinarion for the detection of stenoses of $30 \%$ or more are also plotted. From this ROC-curve it is obvious that the estimation of the degree of spectral broadening is superior to the peak frequency andysis and the indirect techniques in the detection af stenotic lesions.

study corroborate our earlier findings on spectral analysis of the CW Doppler signal of the ICA $(8,9)$.

The direct Doppler examination of the cervical carotid arteries can be expected to differentiate between severe stenosis and total occlusion of the ICA. Therefore, in three cases the results of the direct Doppler examination merit further explanation. In one case no Doppler signal could be obtained from the $\llbracket \mathrm{CA}$, while the Doppler signal of the CCA was compatible with an occlusion of the ICA. In this patient both indirect tests were indicative of a hemodynamically signiticant stenosis on the same side. At arteriography, approximately 48 hours later, no lesion could be detected at the ICA. The repeated noninvasive tests (not included 
in this study) showed no abnormal results. This phenomenon, which has also been met by other investigators, may be caused by vasospastic disorders of the cervical arteries. During surgery the occurrence of vasospasm can sometimes be noiced. In two cases the Doppler examination of the cervical carotid arteries showed a severe stenosis ("Grade 4" partern) of one of the branches of the CCA and total occlusion (absence of the Doppler signal) of the other branch of this artery. In these cases the artery showing severe spectral broadening was inadvertently identified as the ICA. At arteriography it proved to be a total occlusion of the ICA and a sewere stenosis of the ECA in both cases.

Because of basic differences in the Doppler processing technique the value of the $A / B$ ratio of the first and second systolic peak of the Doppler signal of the CCA, as described by Gosling et al $(23,211)$, or the value of the S/D ratio of the CCA, as described by Mol $(198,199)$, cannot be expected to bear a significant relation to the degree of narrowing of the origin of the ICA in this study.

6-7 Results of the direct Doppler examination of the external carotid artery and the common carotid artery

Arteriographic examination of the ECA revealed a stenotic lesion in 47 cases. A Doppler signal showing spectral broadening was recorded in 51 ECAs. The relation between the degree of stenosis of the ECA and the Doppler findings in the ECA is shown in table 6-19. In this study a vast amount of normal ECAs was found: 249 arteries.

TABLE 6-19. Relation between the degree of stenosis of the ECA and the results of the direct Doppler examination in 148 patients (296 sides).

degree of

stenosis

$0-9^{1 \%} \%$

$10-19 \%-$

$20-29 \%$

$30-39 \%$

$40-49 \%$

$50.59 \%$

$60-69 \%$

$70.79 \%$

$80.89 \%$

$90.99 \%$

$100 \%$

cotal

\section{-..- degree of spectral broadenung -.. \\ Grade 1 Grade 2 Grade 3 Grade 4 Grade 5 total}

242

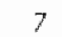

$-$

249

$\begin{array}{rrr}242 & 7 & - \\ \ldots & - & - \\ - & - & - \\ - & 2 & - \\ - & 4 & 1 \\ - & 2 & 5 \\ - & 1 & 6 \\ - & 1 & 1 \\ - & 2 & 6 \\ - & - & 1 \\ 245 & - & - \\ & 19 & 20\end{array}$

$\begin{array}{rrr}- & - & 249 \\ - & - & \\ - & - & 1 \\ - & - & 2 \\ - & - & 7 \\ - & - & 7 \\ - & - & 7 \\ 1 & - & 3 \\ 4 & - & 12 \\ 3 & - & 4 \\ - & 4 & 4 \\ 8 & 4 & 296\end{array}$


The number of stenotic lesions in the ECA is limited. As in the case of the Doppler examination of the ICA the more pronounced degree of spectral broadening is associated with more severe stenotic lesions of the ECA. A Doppler signal of the ECA showing mild spectral broadening was seen in 7 cases in which arteriography revealed no stenosis. On the other hand, a Doppler signal without spectral broadening was obtained from 1 ECA with a stenosis of $20 \%$ to $29 \%$ and from 2 ECAs with a stenosis of $40 \%$ to $49 \%$. The various degrees of spectral broadening are observed in ECAs with a wide range of stenosis. The ability of the direct Doppler examination to recognize stenotic lesions at the origin of the ECA may be important in the analysis of cervical bruits. The sensitivity and the specificity of the direct Doppler examination, derived from the data in table 6-19, are shown in table 6-20.

TABLE 6-20. Sensitivity and specificity of the direc Doppler examination of the ECA, compared to arteriography.

\begin{tabular}{lcc} 
degree of & \multicolumn{2}{c}{ direct Doppler examination } \\
stenosis & sensitivity & specificity \\
$>=10 \%$ & 94 & 97 \\
$>=20 \%$ & 94 & 97 \\
$>=30 \%$ & 96 & 97 \\
$>=40 \%$ & 100 & 96 \\
$>=50 \%$ & 100 & 95 \\
$>=60 \%$ & 100 & 92 \\
$>=70 \%$ & 100 & 90 \\
$>=80 \%$ & 100 & 89 \\
$>=90 \%$ & 100 & 85 \\
$>=100 \%$ & 100 & 84
\end{tabular}

The CCA was examined from the level of the clavicula up to the carotid artery bifurcation. Mild spectral broadening of the Doppler signal of the CCA was seen in 30 cases. A lesion of the CCA of $30 \%$ stenosis or more was seen in 5 of these cases. In a patient with a CCA stenosis of $80 \%$ a moderate degree of spectral broadening was seen. The results of the direct Doppler examinations of the CCA showing spectral broadening are summarized in table 6-21. Mild spectral broading of the Doppler signal of the CCA, without a stenosis, was seen in a large number of patients. The possible explanation for this phenomenon may be found in the angle of the Doppler probe in relation to the CCA, especially in older patients with a deep chesr. Furthermore, the CCA at the right side is sampled in a short segment between two bifurcations. At the left side the CCA is longer and the volocity profile may have become more parabolic, thus influencing the width of the Doppler spectrum. Of the $25 \mathrm{CCAs}$ showing spectral broadening of the Doppler signal without a stenosis or with a stenosis of less than $30 \% 15$ cases were obtained from the right CCA and 10 from the left CCA. It seems that mild or moderate spectral broadening of the Doppler signal of the CCA alone is insufficient for the detection of stenotic lesions in the CCA. 
TABLE 6-21. Relation between spectral broadening of the Doppler signal of the CCA and the presence of stenoses in the CCA.

mild spectral broadening: "Grade 2 "

- no proximal lesion present:

25 cases

- lesion of the innominate artery

- Lesion of the CCA:

$100 \%$ stenosis: 1 case

$30 \%$ stenosis : 2 cases

$70 \%$ stenosis: 1 case

$80 \%$ stenosis: 1 case

moderate spectral broadening: "Grade 3":

- lesion of the CCA:

$80 \%$ stenosis : 1 case.

6-8 Discussion on the results of the noninvasive tests, regarding stenotic lesions in the Internal Carotid Artery.

The recommendation to use a specific test as screening for the detection of stenotic lesions of the extracranial cerebral arteries must he based on the ability of the test to recognize "diseased" arteries and to exclude "healthy" arteries.

The observed differences in sensitivity and specificity of the various indirect noninvasive tests were not statistically significant. Therefore, on that basis no indirect test can be preferred to one of the others. If used merely for the detection of stenotic lesions in the cerebropetal arteries the periorbital Doppler examination is as useful as the OPG-Gee, but has less contraindications. The periorbital Doppler examination could be performed in all but 1 of the patients, whereas the OPG-Gee could not be performed or interpreted in 14 patients! On the other hand, the OPG-Gee provides the investigator with a numerical result (ratio OAP/BAP) and allows a more accurate determination of the efficacy of the collateral circulation.

In the $\mathbb{R O C - c u r v e ~ s h o w n ~ i n ~ f i g u r e ~ 6 - 9 ~ t h e ~ s e n s i t i v i t y ~ a n d ~ t h e ~ s p e c i f i c i t y ~ o f ~ t h e ~}$ various direct and indirect rests are shown. From figure 6-9 it is obvious that the direct Doppler examination combines the highest sensitivity with the smallest loss in specificity for degrees of narrowing of $30 \%$. At this degree of stenosis the differences between the indirect tests and the direct Doppler examination are statistically significant $(p<0.001)$. Therefore, the direct Doppler examination is to be preferred for the noninvasive evaluation of the cervical carotid arteries. The accuracy of the noninvasive tests is dependent on the prevalence of disease in the investigated population (208). In this study the diagnostic accuracy was corrected 


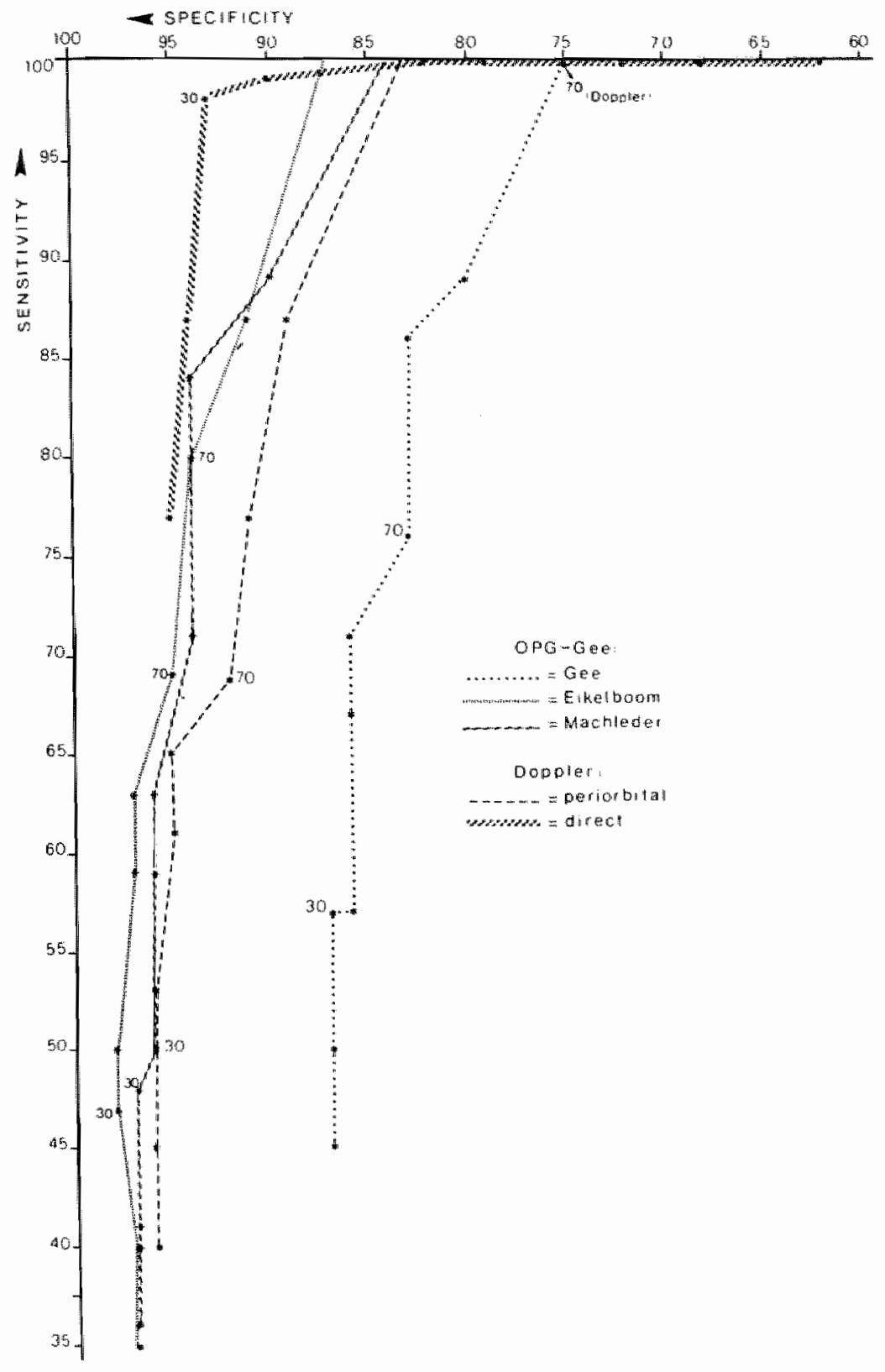

Figure 6-9. ROC-curve showing the relation between the sensitivity and the specificity of the various noninvasive tests in the detection of stenotic lesions of the internal carotid artery if the threshold for "stenosis" is increased in steps of $10 \%$. From this curve it is obvious that the direct Doppler examination reaches the highest sensintivity in combination with the highest specificity for lesions of $30 \%$ dianeter reduction or more. 
for by using Kappa (chapter 5). The Kappa-corrected diagnostic accuracy of the indirce noninvasive tests, if a stenosis of $70 \%$ diameter reduction is used as a threshold, is $0.54,0.68,0.68$ and 0.62 for the OPG-Gee (Gee), OPG-Gee (Eikelboom), OPG-Gee (Machleder) and the periorbital Doppler examination, respectively. The Kappa-corrected accuracy of the direct Doppler examination if the five categories of spectral broadening are used in comparison with five different levels of stenosis at the origin of ICA is 0.80 :

degrece of

stenosis

$0 \%-29 \%$
$30 \%-49 \%$
$50 \%-69 \%$
$70 \%-99 \%$
$100 \%$
total

\begin{tabular}{|c|c|c|c|c|c|}
\hline \multicolumn{5}{|c|}{-- degret of spectral broadening --- } & \\
\hline Gradic 1 & Grade 2 & Grade 3 & Grade 4 & Grade 5 & tot \\
\hline 168 & 12 & 0 & 0 & 1 & 10 \\
\hline 2 & 22 & 2 & 0 & 0 & \\
\hline 0 & 0 & 18 & 3 & 0 & \\
\hline 0 & 2 & 12 & 32 & 0 & \\
\hline 0 & 0 & 0 & 2 & 20 & \\
\hline 170 & 36 & 32 & 37 & 21 & \\
\hline
\end{tabular}

In this latter approach the spectral broadening of the direct Doppler examination, without taking the peak systolic frequency shift into consideration, is not only used to detect stenotic lesions in the origin of the ICA (either spectral broadening or not) but also to quantify the degree of stenosis (Grade 1 versus $0 \%-29 \%$; Grade 2 versus $30 \%-49 \%$; and so on). Evern then the corrected diagnostic accuracy is better than that of any of the indirect tests.

In conclusion, the direct Doppler examination of the origin of the ICA is superior to indirect noninvasive tests in the detection of stenoric lesions of the ICA. For the quantification of the overall collateral circulation the $O P G-G e e$ may be preferred, as it also takes the collateral circulation through the circulus arteriosus into consideration. 


\section{GENERAL DISCUSSION}

From the results in the present study it is obvious that the direct noninvasive test is superior in the detection of stenotic lesions of the internal carotid artery as compared to the indirect rechniques. The difference between the direct Doppler examination and the indirect techniques in the detection of lesions with a stenosis of $30 \%$ or more is statistically significant $(p<0.001)$. Stenotic lesions of the internal carotid artery with a degree of narrowing of $30 \%$ or more almost invariable showed spectral broadening of the continuous wave (CW) Doppler signal. On the orher hand spectral broadening was seen in only a very limited number of CW Doppler signals obtained from internal carotid arteries without a stenosis. Furthermore, the degree of spectral broadening of the direct $\mathrm{CW}$ Doppler examination allows a fair estimation of the degree of stenosis of the lesions and, in contrast to the indirect techniques, it can differentiate between high grade stenosis and total occlusion of the artery under investigation. The ability of the direct CW Doppler examination to detect stenotic lesions in the extracranial cerebropetal arteries cannot be improved by combining this technique with the results of the indirect methods. However, the hemodynamic consequences of the stenosis can be monitored far better by the indirect techniques. Both the detection of collateral circulation and the detection of a pressure drop, as caused by the stenosis, are the sole domain of the indirect techniques. Obviously, the direct and the indirect techniques are complementary. Therefore, a noninvasive examination of the extracranial cerebral circulation is incomplete if either the direct or the indirect technique are left out. The direct CW Doppler examination detects the degree of blood flow disturbance, as caused by a stenosis. The periorbital Doppler examination measures specifically the presence or absence of collateral circulation from the external carotid artery to the internal carotid artery. The ocular pneumoplethysmography (OPG-Gee) reveals the resultant of the effects of the stenotic lesion and collateral circulation. Both the occurrence of a pressure drop across a stenosis and the utilization of the anatomical pathways of collateral circulation are limited to lesions with a severe degree of cross-sectional area reduction. In theory the resultant pressure in the ophthalmic artery can be maintained at a sufficiently high level (normal OPGGee) through adequate collateral circulation from the external carotid artery to the internal carotid artery (abnormal periorbital Doppler examination) and/or through the intact circle of Willis. This phenomenon might account for a larger 
number of 'positive" periorbital Doppler examinations, as compared to the number of 'positive' OPG-Gee cxaminations in the presence of severe stenotic lesions of the intermal carotid artery. In the present series this assumption could not be confirmed because of the limited number of lesions in the range from a non-hemodynamically significant stenosis to a themodyanically significant one.

The performance of the OPG-Gee requires no special skill. The OPG-Gee examination provides the ophthalmic artery pressure in a quantitative way. Registrations of the OPG-Gee are shown in figure 4-10. The most common interruption in these pattern is caused by blinking of the eyes. This artifact is easily recognized. The OPG-Gee examination cannot be performed in all patients. In this study the OPG-Gee could not be performed in 12 patients $(8.1 \%)$ and could not be interpreted in another 2 patients $(1.4 \%)$. Therefore, incomplete results of the OPG-Gee examination were present in $9.5 \%$ of the patients examined in this series. Medical contrandications precluded the use of the OPGGee by Lynch et al (173) in 8 out of 75 patients $(10.7 \%)$. The results of the OPGGee, as presented in this study, support the findings in other studies $(11,186,210,220)$. As suggested by Eikelboom (75) the selection of the criteria for the interpretation of the OPG-Gee may depend on the patient population. If the test is used to screen a 'healthy' population for the prevalence of carotid artery disease the test should have a high specificity. If the test is used to screen a population of patients with symptoms of cerebral ischemia for the prevalence of carotid artery disease the test should have a high sensitivity. The differences in the sensitivity and the specificity of the OPG-Gee if the cricersa according to Gee are used on the one hand, and the criteria according to Eikelboom and Machleder on the other hand are not statistically significant $(0.10>\mathrm{p}>0.05)$. In this study the absolute value of the ophthaimic artery pressure and the amplitude of the OPGGee signal did not provide any additional information for the differentiation between normal and stenotic segments of the cerebropetal arteries. This was also observed by Eikelboom (75) concerning the amplitude of the OPG-Gee signal.

The use of the periorbital Doppler examination requires experience and careful interpretation of the results of the test, even during the examination. The importance of the angle of insonation of the periorbital artery is emphasized. Furthermore, the periorbital Doppler examination should be performed without exerting any pressure to the underlying artery. No limitations for the performance of the test are known. In this study the results of the periorbital Doppler examinacion could not be interpreted in $\mathbb{1}$ patien $(0.7 \%)$. The results of the periorbital Doppler examination obtained in this study are in agreement with the results obtained in other studies $(101,143,172,185)$. The periorbital Doppler examination may yield a higher sensitivity if the supraorbital artery is insonated (212). The most important disadvantage of the periorbital Doppler examination, as compared to the OPG-Gee, is the lack of quantification. The examination is "positive", 'negative' or "not interpretable'. This reduces the value of the perior- 
biral Doppler examination in follow-up studies on extracranial cerebropetal atherosclerosis. The periorbital Doppler examination can be performed with the CW Doppler instrument, also needed for the direct Doppler examination.

In the direct CW Doppler examination the differentiation berween the Doppler signals arising from the external carotid artery and the internal carond artery is relatively easy (chapter 4). It is facilitated by the audiovisual interpretation of the signals. In the external carotid artery the separation of the systolic and the diastolic phase by the decreased flow velociries, seen as a notch on the sonograms, is of special help up to high grade lesions. In figure $7-1$ this notch is shown in sonograms obtained from external carotid arteries with various degrees of narrowing. In sonograms obtained from the internal carotid artery this notch is almost invariably absent or far less prominent. In the sonogram of the common carotidartery the notch between the systolic and the diastolic part is intermediare, as compared to the notch in the sonograms of the internal carotid artery and the external carotid artery (figure 4-5). Furthermore, it is possible to change the Doppler signal of the external carotid artery by compressing the branches of this artery, and thus changing the peripheral resistance of the vascular bed of the artery. The sonogram of the carotid artery bifurcation is characterized by the occurtence of various, lower frequencies and negative frequencies (figure 4-4). Thiscan be explained by decelaration of the arterial flow due to an increase in total cross-sectional area at this bifurcation and the presence of backflow with helical vortices in the carotid bulb $(231,232)$. In the sonogram certain characteristics, caused by a stenosis, can be observed. Some of these changes were not anticipated at the start of this study and, therefore, not taken into consideration as possible criteria for the detection of vascular lesions. In short some of these characteristics will be mentioned. Firstly, the upslope of the systolic part of the sonogram is steep if no 'significant' lesion is present proximal to the site of sampling in the artery. Distal to a significant lesion the upslope becomes more gradual. Sometimes the appearance of a 'lazy-s' configuration in the upslope can be seen distal to a stenotic lesion (figure 7-2). Secondly, immediately proximal to and at the entrance of a stenosis 'wall thumping' can sometimes be noticed early in systole. An example of this low-Frequency and high-amplitude movement of the arterial wall and its effect on the sonogram is shown in figure 4-8B. Because of wall thumping the upslope of the systolic part of the cardiac cycle is sometime less conspicuous (226).

Spectral broadening of the CW Doppler signal reflects the presence of a variety of flow velocities. The presence of multiple flow velocities over the cross-sectional area of the vessel is a physiological observation. The increase in mean flow velocity will result in a wider range of flow velocities. The flow velocity in the arterial system may be increased because of a stenosis. Such a stenosis can be caused by a lesion in the arterial wall, by external compression of the arterial wall or by elongation of the artery. Furthermore, increased blood flow velocities can 

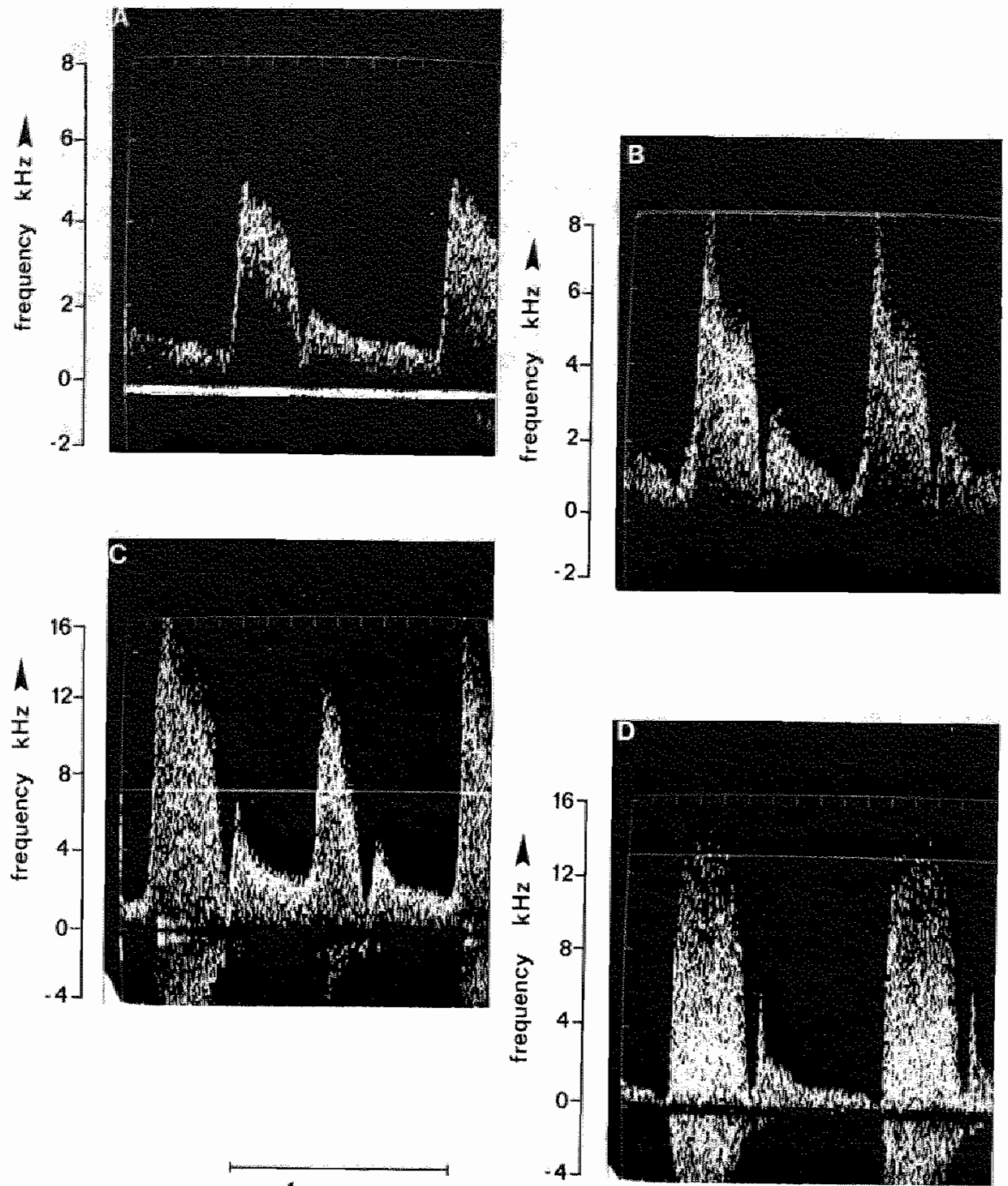

$1 \mathrm{sec}$.

time $=$

Figure 7-1. Examples of the sonogtams of the continous-wave Doppler signals obtained from external carotid arteries showing various degrees of stenosis. In the sonograms the differences in the degree of spectral broadening can be seen. Even in the presence of a severe stenotic lesion of the external carotid artery, characterized by severe spectral broadening (D) the deflection between the systolic part and the diastolic part of the cardiac cycle is obvious. 

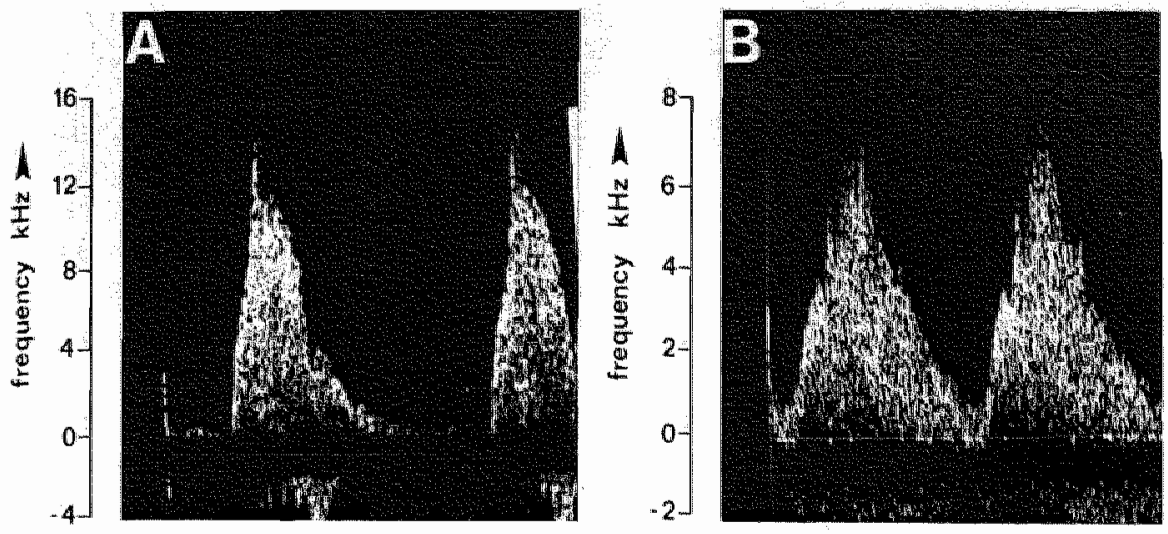

$1 \mathrm{sec}$

time

Figure 7-2. Sonograms of the continous-wave Doppler signal of the common carocid artery showing the "lazy-s" configuration of the upslope of the systolic phase (A) and the more gradual upslope (B). These phenomena ean be seen in the presence of a more proximal lesion of the common carotid artery.

be seen in case of arterio-venous malformations, high cardiac output states and lowering of the peripheral resistance in the vascular bed. Besides, the width of the Doppler spectrum depends on the angle of insonation and the site of insonation. For example, mild spectral broadening of the Doppler signal of the common carotid artery, without a detectable stenosis on the arteriogram, was seen in a large number of patients. Elongation of the artery can be diagnosed by trying to follow its course, and adjusting the angle of insonation. The use of a B-mode imaging technique may be useful in the investigation of elongated arteries.

The apprectation of the direct CW Doppler examimation is based on the sound of the Doppler signal, the peak systolic frequency shift and the wid th of the Doppler spectrum. The interpretation may require repeated investigations of the site of the lesion, from different angles of insonation. The entire arterial pathway from low in the neck up to the angle of the mandibula is in sonated. "The decision whether changes in the sonogram are representative for a stenocic lesion of not is made after the examination of the common carotid artery, external carotid artery and the internal carotid artery. The peak systolic frequency shift of the Doppler signal could be reliably reproduced on consecutive investigations (83). This relationship berween increased peak systolic Doppler frequency shifts and stenoses of the internal carotid artery has been well established, and proven to be a rellable indication of diameter reduction $(14,18,24,125)$. Johnston (126) states that, at the 
present time, the measurement of the peak systolic frequency shift from the recordings of the Doppler signals remains the most common method for quantitarive analysis.

However, determination of the peak systolic frequency of a Doppler signal depends on the exact insonation of the site of stenosis and varies with the angle of insonation. Therefore, for the evaluation of stenosis in follow-up studies the information provided by the peak systolic frequencies must be interpreted, bearing these limitations in mind. On the other hand, the interpretation of the degree of spectral broadening of the Doppler signal remains subjective and open for discussion. Even if the classification according to Barnes is used the transition from one category of spectral broadening to the other is controversial, except if absence of the Doppler signal is observed. The degree of spectral broadening, caused by a stenosis, depends on the phase of the cardiac cycle, the site of sampling, the shape of the stenotic lesion, and the severity of the stenosis.

Attempts have been made to quantify the degree of spectral broadening of the CW Doppler signal $(64,130,137,235)$. From some in vitro studies promising results have been obtained. However, the real value of the objective assessment of the degree of spectral broadening in the detection of minor and moderate lesions remains to be seen. The decision to allot the findings of the direct Doppler examination to a certain category was based upon subtle changes in the Doppler spectrum found at a single site along the course of the artery. The translation of the findings of the direct Doppler examination into an estimation of a degree of stenosis is based on the degree of spectral broadening and the maximal frequency shift of the Doppler signal that can be detected at the site of the lesion.

The mere finding of spectral broadening of the Doppler signal requires in addition an assessment of the probable cause. The ability of the investigator to perform this extension of the Doppler examination determines the ultimate diagnostic value of the technique in the detection of stenotic lesions.

For example, in figure 6-6 the lesion indicated by - A-can be recognized as a severe stenotic lesion because of the severe spectral broadening even though the peak systolic frequency shift does not represent the high blood flow velocity expected at the site of such a lesion. Also the lesions indicated by - B-can be recognized as severe stenotic because of the very high frequency shifts ( $18 \mathrm{kHz}$ with a Doppler probe with an emission frequency of $8 \mathrm{MHz}$ ) although the degree of spectral broadening is moderate ("Grade 3 "). The peak systolic frequency shift should be assessed in the narrowest part of the stenosis. The degree of spectral broadening should be assessed immediately distal to the stenosis. In figures 7-3,7-4 and 7-5 examples are shown of the sonograms of the continuous wave Doppler signals obtained from the carotid arteries of some patients. The need to further quantily the findings of the Doppler examinations is obvious and has been recognized by various investigators $(15,125,150,235)$. This problem has not yet been solved. 
In comparison to the continuous-wave Doppler systems the pulsed Doppler systems may have advantages over the CW Doppler systems in the detection of lesions of a lesser degree of stenosis $(29,110)$. However, the detection of flow disturbances due to minor stenotic lesions at an arterial bifurcation is complicated by the occurrence of flow disturbances induced by the division of blood flow at the bifurcation which mimics the 'stenoric' flow disturbances $(1,231,232)$. The advantages of the multi-gated pulsed Doppler systems over the single-gated pulsed Doppler systems have been described in detail by Reneman et al (232). Even with the former systems the complicated flow patterns found at the bifurcation of the common carotid artery limit the diagnosis of minor $(<30 \%$ diameter reduction) to moderate ( $<50 \%$ diameter reduction) lesions. The diagnosis of these lesions is based on secondary changes, such as the presence of recirculation at a site where it is not expected or diminished vessel wall displacement (232).

Surgical treatment of extracranial cerebrovascular disease may be necessary in some cases and justified in others. The treatment of extracranial cerebrovascular disease should be based primarely on the presence of functional disturbances, and not on anatomical lesions. Arteriography presents the anatomical features of the cerebropetal circulation. It has been the "gold standard" for the evaluation of the arterial system for years. The introduction of digital subtraction angiography has enabled the visualization of the arterial tree with minimal risk and in less time. The poor contrast density is responsible for a large number of inadequate studies if the contrast medium is injected intravenously (68). Nevertheless, this technique was found to be satisfactory compared to B-mode imaging (290) or operative findings (247). In comparison to conventional arteriography or digital subtraction angiography with intra-arterial contrast injections the intravenous studies have increasingly demonstrated inconsistent results $(181,223)$. As the intravenous digital suburaction angiography shows a tendency to underestimate the degree of stenosis of a lesion in the carotid arteries $(6,8,239)$ the rechnique should not be used for the screening of patients (98). Arteriography and noninvasive studies provide different information. These differences should be appreciated. The indication to perform arteriography may not be related to cerebral ischemia. However, if arteriography is performed to evaluate the extracranial cerebropetal arteries it should only be used after the noninvasive examination has been performed to evaluate the results of the noninvasive studies. The noninvasive examination of the extracranial cerebropetal arteries reflects physiological phenomena of the circulation in these arteries.

From the results in the present study it is obvious that noninvasive tests can, and should, be used to assess the presence and severity of lesions in the extracranial cerebropetal arteries. Since the noninvasive tests are accurate, virtually without risk and can be repeated without limitation they also provide a reliable method for the follow-up evaluation of lesions of the extracranial cerebropetal arteries. 

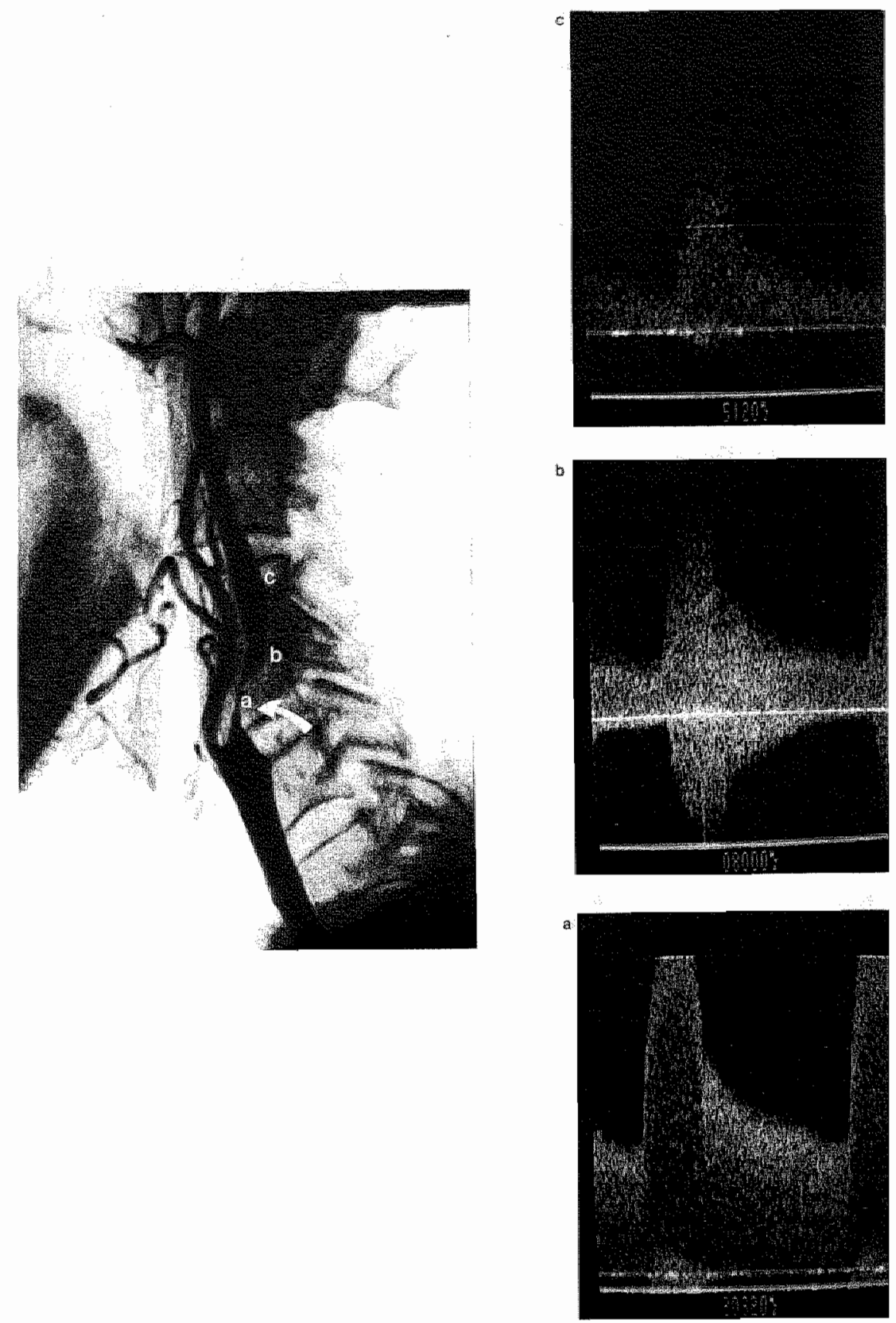

Figwre 7-3. Sonogram of the continuous-wave Doppler signal of the internal carotid artery obtained at the site of a septal stenosis, immediately distal to this lesion and at sonne distance from this lesion. The spectral brondening of the Doppler signal is most obvious immediately distal to the stenosis. 

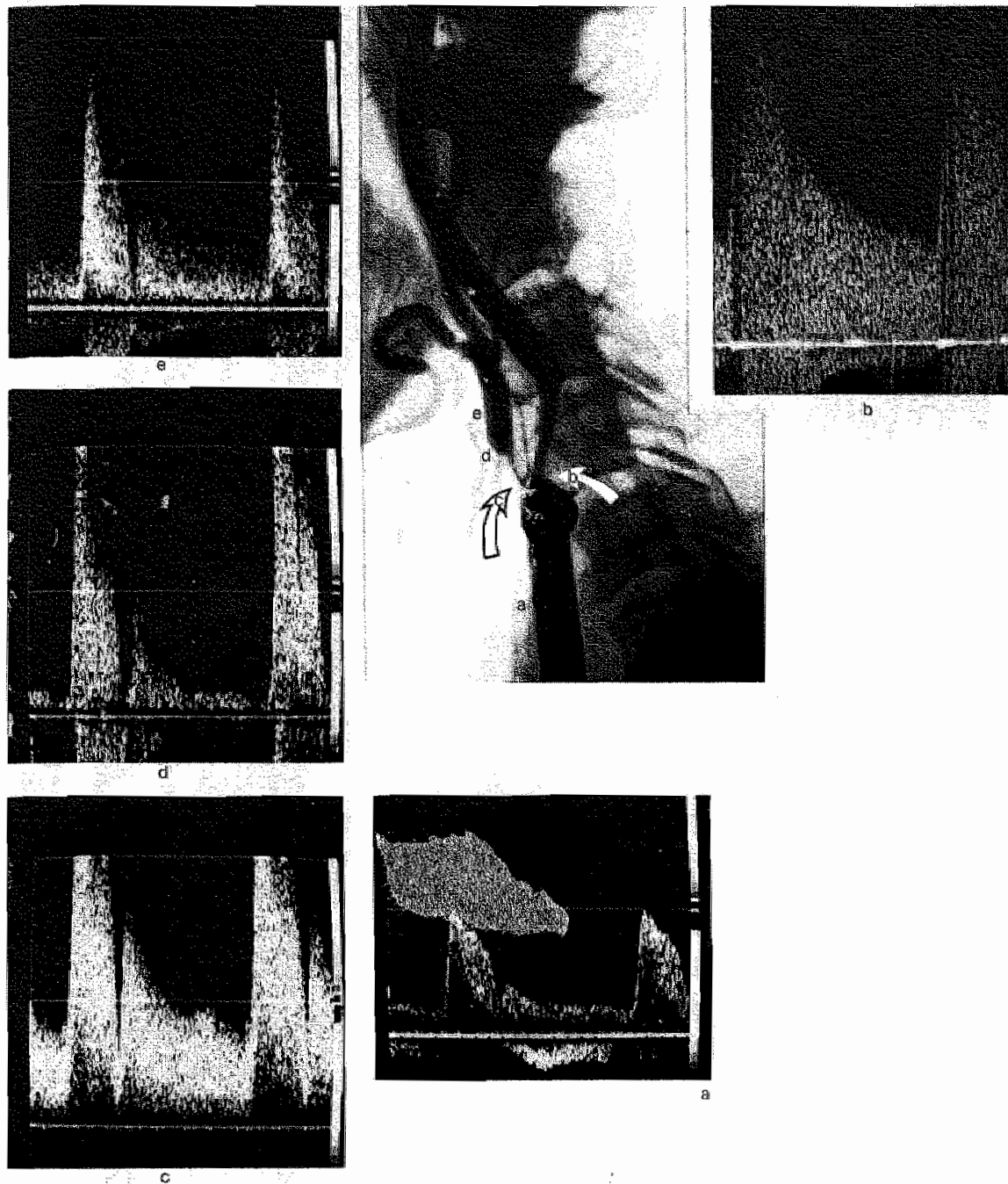

Figure 7-4. Sonograms of the continuous-wave Doppler signals of the internal carotid artery and the external carotid artery showing spectral broadening caused by the severe stenosis in these arteries. 


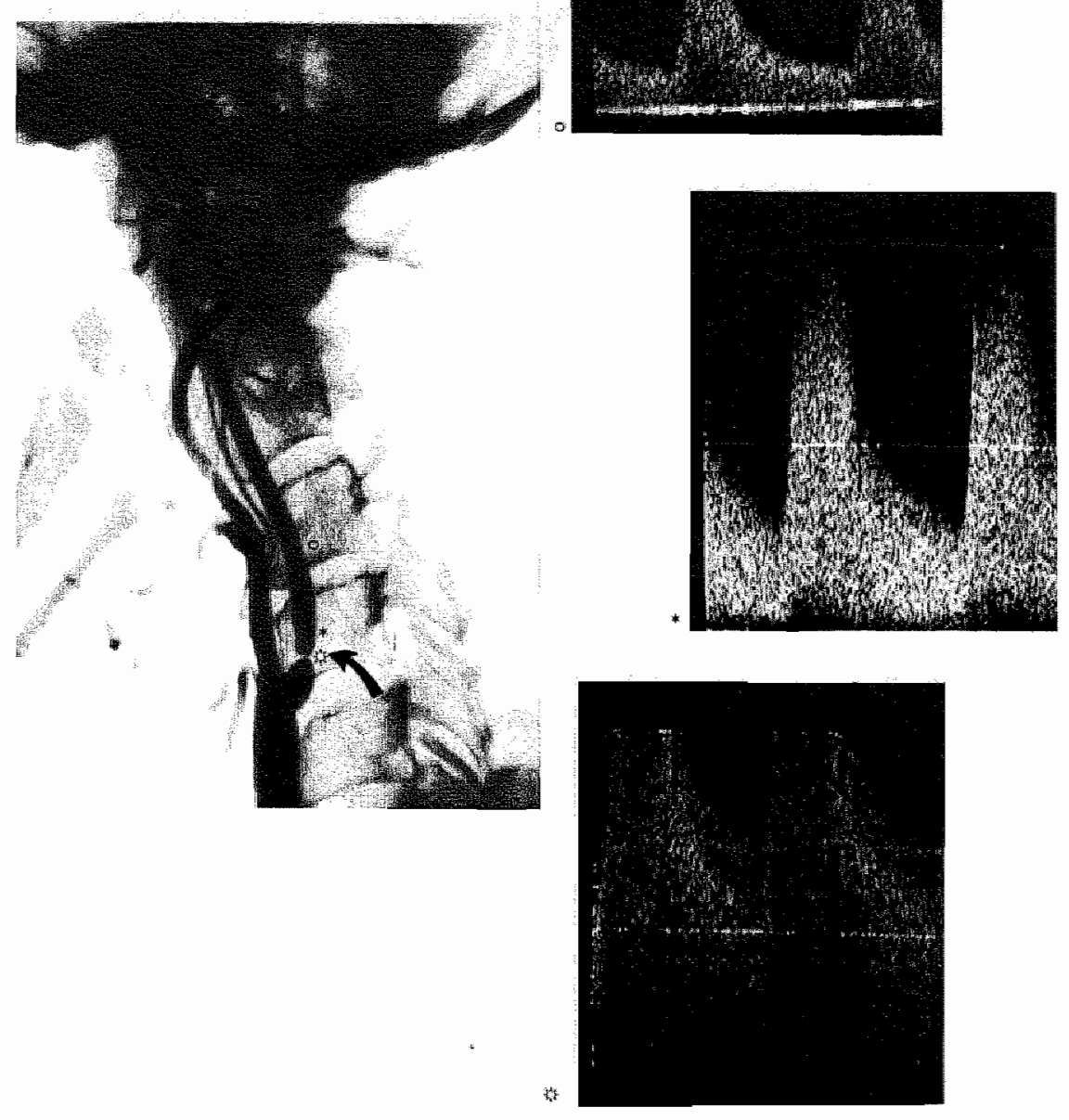

Figure 7-5. Sonograms of the continuous-wave Doppler signal of the internal carotid artery showing various degrees of spectral broadening and peak frequency shifts at the different sites in the stenotic lesion. 


\section{SUMMARY}

In the introduction the need for noninvasive examination of the cervical arteries is explained and the objective of the study is described.

In chapter 1 the anatomy and the physiology of the cerebral circulation are described. The collateral circulation, important in the presence of stenoses in the cerebropetal arteries, can be differentiated in extracranial, extracranial to intracranial, and intracranial collateral circulation.

In chapter 2 the presence of atherosclerotic lesions in the extracranial cerebropetal arteries is described. The atherosclerotic lesions may have a rough and irregular surface, and are then called 'ulcerated'. Ulcerated lesions are not always diagnosed. The detection of ulceration in the stenosis depends on the method used for the evaluation of the lesion. Stenotic lesions can cause cerebral ischemia by shooting emboli or by interference with blood flow through the diseased artery, especially if the collateral circulation fails. Indications are found that both mechanisms occur, and these indications are discussed.

In chapter 3 the basic principles that control blood flow in arteries are described, as far as they are related to the noninvasive examination of atherosclerotic lesions of the cervical arteries. Blood flows as a result of local differences in total energy. Total energy of the blood can be thought to be composed of hydrostatic energy, kinetic energy and potential energy. Kinetic energy and potential energy can change from one form into the other. The blood flow velocities over the crosssection of a pipe will fully develop in a long straight pipe and take the form of a parabola, in which the flow velocity in the centre of the pipe is higher than the flow velocity at the wall. Then various flow velocities will be present at the same time. Ar stenoses and bifurcations in the arterial system disturbances of this laminar flow pattern will occur. In the case of disturbance of the laminar blood flow a multitude of blood flow velocities may occur and turbulence may appear. The disturbance of laminar blood flow will cause the loss of potential and kinetic energy.

In chapter 4 the methods of examination to detect lesion in the cervical arteries are described. The examination can be performed at the site of the carotid artery or at some distance of these arteries. Examinations at the site of the carotid artery attempt to detect local changes in the vessel wall (imaging techniques) or in the blood flow velocities (direct Doppler techniques). In the detection of disturbaces of the blood flow velocities the Doppler flowmeter is generally used (see below). 
These methods are called "direct" methods of examination. In the examinations at some distance of the cervical arteries attempts are made to establish the consequences of changes in the cervical arteries. These methods are called "indirect" methods. The consequences of changes in the cervical arteries can be observed as a decrease of the blood pressure distal to the stenosis or the delay in the arrival of the pulse wave (OPG-Kartchner). The decrease in the blood pressure can be measured as a real decrease in the blood pressure in the ophchalmic artery. This blood pressure can be assessed at the bulb of the eye using the ocular pneumoplethysmography (OPG-Gee). The decrease of the blood pressure distal to the carotid artery can al so elicit periorbital collateral circulation. The presence of this collateral circulation can be investigated with the periorbital Doppler examination. Because these methods of examination try to investigate characteristics from the outside they are called 'noninvasive'. The most commonly used direct and indirect methods of examination are described. Furthermore, the contrast arteriography is discussed. In this technique an image of the arteries is obtained by the local injection of a contrast medium. As indicated above the Doppler ultrasound examination is important in this noninvasive diagnosis. The Doppler examination is based on the frequency shift of sound if it is reflected from moving particles. The reflection from fast moving particles will cause a greater frequency shift than the reflection from slowly moving particles. In the Doppler examination a continuously emirting signal (continuous wave Doppler system) or an intermittently emitting signal (pulsed Doppler system) can be used. The changes in the blood flow velocity, caused by local stenoses, can be recognized by the frequency shift of the Doppler signal. The Doppler signal can be interpreted in various ways. The audio interpretation of the Doppler signal is often used. It lacks the documentation of the information obtained. In the analogue interpretation of the Doppler signal the various frequencies of the Doppler signal are represented by a single curve. In spectral frequency analysis of the Doppler signal these various frequencies are represented separately in the Doppler spectrum. Especially in the presence of many different frequencies in the Doppler spectrum, as with disturbances of the laminar blood flow, this may prove to be an advantage. In the present study a continuous wave Doppler flowmeter with spectral frequency analysis of the Doppler signal was used.

In chapter 5 the methods of examination used in this study, and the criteria used for the interpretation of the results, are described. As 'golden standard' the contrast arteriography was used. Using local injections of contrast medium images of the arterial tree were obtained. These $\mathrm{X}$-rays were made using simultaneous bi-plane projections. The estimation of the degree of narrowing of the artery is described. As noninvasive methods of examination the OPG-Gee, the periorbital Doppler examination and the direct Doppler examination of the cervical carotid artery were used. In the OPG-Gee examination the results were interpreted with the criteria according to Gee, Eikelboom and Machleder. In the periorbital Doppler examination the direction of flow in the periorbital arteries and the peak frequency shift were assessed. In the direct Doppler examination the 
width of the Doppler spectrum and the amount of the frequency shift were estimated. The results of the noninvasive methods of examination were compared with the results of contrast arteriography. The chapter concludes with the description of the method of analysis of the results.

In chapter 6 the results of the present study are described. Some characteristics of the patient population are provided. The results of the contrast arteriography are described regarding the site and the severity of the stenoses. Because the indirect methods of examination reflect information on a larger traject of the arterial tree the concept of 'arterial segment' is introduced. This arterial segment is the traject from the aortic arch to the ophthalmic artery. As degree of stenosis, for comparison with the indirect methods of examination, the most severe degree of diameter reduction in this traject is taken. The results of the OPG-Gee, the periorbital Doppler examination and the direct Doppler examination with spectral frequency analysis of the Doppler signal are described. Subsequently, the results are discussed and compared with those obtained from other studies. Especially the results of the noninvasive techniques regarding the detection of stenotic lesions in the internal carotid artery are discussed. It proves that the $O P G-G e e$ in the detection of stenotic lesions of $30 \%$ diameter reduction or more if interpreted according to Gee, Eikelboom and Machleder has a sensitivity of $57 \%, 47 \%$ and $48 \%$, respectively. The specificity of the OPG-Gee is $87 \%, 98 \%$ and $97 \%$, respectively. The periorbital Doppler examination, with this degree of diameter reduction, has a sensitivity of $50 \%$ and a specificity of $96 \%$. The direct Doppler examination, with stenoses of $30 \%$ diameter reduction or more, has a sensitivity of $98 \%$ and a specificity of $93 \%$. If the threshold separating 'healthy' from 'diseased' is increased to $70 \%$ diameter reduction the sensirivity of the OPG-Gee interpreted with the criteria according to Gee, Eikelboom and Machleder is $76 \%$, $69 \%$ and $71 \%$, respectively. The specificity of the examination is then $83 \%, 95 \%$ and $94 \%$, respectively. In this situation the sensitivity and the specificity of the periorbital Doppler examination is $69 \%$ and $92 \%$, respectively. Then the direct Doppler examination has a sensitivity of $100 \%$ and a specificity of $75 \%$. It proves that the noninvasive examination of the extracranial cerebral circulation is accurate in the detection of stenoses at the bifurcation of the carotid artery. Even stenoses with a moderate degree of diamerer reduction can be detected. The direct noninvasive method of examination is more accurate in the detection of these lesions than the indirect methods of examination. However, this does not derogate the value of the indirect methods of examination from their ability to investigate the hemodynamic consequences of a stenosis. Therefore, the indirect methods can be seen as an addition to the direct method. The drawback of the indirect methods remains the impossibility to distinguish total occlusion from severe stenosis of the cervical arteries.

Chapter 7 provides a general discussion on the methods of examination. The methods are compared as far as accuracy and limitations are concerned. The questions raised in the introduction are answered. The tendency to evaluate stenoses in the carotid arteries with noninvasive methods of examination instead of contrast arteriography is explained. 


\section{SAMENVATTING}

In de inleiding wordt de noodzaak van niet-invasief onderzoek van de halsslagaders toegelicht en wordt de vraagstelling van de studie beschreven.

In hoofdstuk 1 worden de anatomie en fysiologie van de cerebroperale circulatie beschreven. De collaterale circulatie, van belang bij de aanwezigheid van vernauwingen (stenosen) in de cerebropetale circulatie, kan worden onderscheiden in een extracraniele-, een extra- naar intracraniele- en een intracraniele collaterale circulatie.

In hoofdstuk 2 wordt het voorkomen van atherosclerotische letsels in de extracraniele vaatboom beschreven. De atherosclerotische letsels kunnen een ruw en onregelmatig oppervlak hebben, en worden dan "ulcera" genoemd. Ulcera worden niet altijd gediagnostiseerd. Het ontdekken van een ulcus in een vernauwing is afhankelijk wan de methode warmee het onderzoek wordt verricht. Vernauwingen van de slagaders kunnen cerebrale ischemie veroorzaken door embolisatie van partikels of door belemmering van de bloedstroom in de aangedane slagader, vooral wanneer de collaterale circulatie te kott schiet. Voor het bestaan van deze beide mechanismen kunnen aanwijzingen worden gevonden en deze aanwijzingen worden besproken.

In hoofdstuk 3 worden de basale principes, die ten grondslag liggen aan de stroming in slagaderen, besproken voor zover deze van belang zijn voor de nietinvasieve diagnostiek van arherosclerotische lesies in de halsslagader. Het bloed stroomt als gevolg wan locale verschillen in totale energie. De totale energie van het bloed kan beschouwd worden te zijn opgebouwd wit hydrostatische energie, kinetische energie en potentiele energie. Deze twee laatste vormen van energie kunnen in elkaar overgaan. De snelheidsverdeling van de bloedstroom zal in een lange rechte buis zich volledig ontwikkelen en de vorm van een parabool aannemen, waarbii de stroomsnelheid in het centrums wan de buis hoger is dan de stroomsnelheid aan de wand van de buis. Dan zullen dus meerdere stroomsnellheden gelijkrijdig voorkomen. Bij stenosen en bifurcaties in het arteriele systeem treedt een verstoring op van de gelijkmatige bloedstroom. Bij deze verstoring kunnen meerdere stroomsnelheden optreden en wervelingen tot ontwikkeling 
komen. Deze verstoring van de gelijkmatige bloedstroom gaat gepaard met verlies van kinetische en potentiele energie.

In hoofdstuk 4 worden de methoden van onderzoek die letsels in de halsslagader kumnen opsporen besproken. Dit onderzoek kan verricht worden ter plaatse van de halsslagader of op enige afstand. Bij de onderzoeken ter plaatse van de halsslagader wordt getracht om platselijke veranderingen in de vaatwand (imaging technieken) of in de stroming van het bloed (direct Doppler onderzoek) op te sporen. Bij het opsporen van verstoringen in de bloedstroom wordt in het algemeen gebruik gemaakt van Doppler technieken (zie beneden). Deze methoden worden 'directe' onderzoekstechnieken genoemd. Bii de onderzoeken op enige afstand van de halsslagader wordt geprobeerd de gevolgen van de veranderingen in de halsslagader te vast te stellen. Deze methoden worden 'indirecte" methoden genoemd. De gevolgen van een vernauwing in de halsslagader kunnen zich uiten als een vermindering van de bloeddruk stroomafwaarts van de vernatwing, of het vertraagd optreden van een polsgolf (OPG-Kartchner). De verlaging van de bloeddruk kan worden gemeten als een werkelijke vermindering van de bloeddruk in de arteria ophrhalmica. Deze bloeddruk kan worden gemeten aan de oogbol met de methode van 'ocular pneumoplethysmography' (OPG-Gee). De vermindering van de bloeddruk stroomafwaars in de halsslagader kan ook collaterale circulatie in de bloedvaten rond het oog veroorzaken. De anwezigheid van deze collaterale circulatie kan worden onderzocht met het periorbitaal Doppler onderzoek. Omdat deze onderzoeksmethoden van buiten af karakteristieken in het lichaam proberen te objectiveren, worden zij "niet-invasieve" methoden genoemd. De meest gebruikte directe en indirecte methoden van onderzoek worden beschreven. Daarnaast wordt de contrast angiografie besproken, waarbij door middel van contrast injecties in de bloedbaan een afbeelding van de bloedbaan kan worden verkregen. Zoals hierboven aangegeven speelt het Doppler ultrageluid onderzoek een belangrijke rol bij de niet-invasieve diagnostiek. Het Doppler onderzoek is gebaseerd op de verandering in frequentie die het geluid ondergat als het wordt weerkaatst door bewegende voorwerpen. De weerkaatsing door snel bewegende voorwerpen zal een grotere frequentie verandering veroorzaken dan de weerkaatsing door langzaam bewegende voorwerpen. $\mathrm{Bij}$ het Doppler onderzoek kan gebruik worden gemaakt wan een continue (continuous wave Doppler systeem) of van een intermitterende (pulsed Doppler systeem) uitzendbron. De veranderingen in de stroomsnelheid, die her gevolg van locale vernauwingen zijn, kunnen worden herkend aan de frequentie veranderin gen van her Doppler signaal. Her verkregen Doppler signaal kan op verschillende wijzen worden beoordeeld. De interpretatie van het Doppler signaal op gehoor wordt dikwijls gebruikt. Er bestaat dan geen documentatie van de verkregen informatie. Bij de analoge verwerking van het Doppler signaal worden de werschillende componenten van het Doppler signaal gemiddeld en als een enkele curve weergegeven. Bij spectraal analyse van her Doppler signaal worden de verschillende componenten van het signaal apart weergegeven in het Doppler 
spectrum. Met name bij de aanwezigheid van veel verschillende frequenties, zoals bij verstoringen van de gelijkmatige bloedstroom, kan dit een voordeel betekemen. In de huidige studie werd gebruik gemaakt van een continuous wave Doppler flowmeter met spectraal analyse van her Doppler signaal.

In hoofdstuk 5 worden de methoden van onderzoek die in deze studie zijn gebruikt, en de criteria die werden gehanteerd bij het interpreteren van de resultaten beschreven. Als 'gouden standaard' werd de contrast angiografie gebruikt. Met behulp van lokale injecties van contrast in de vaatboom werden afbeeldingen verkregen van de halsslagader. Hierbij werden gelijktijdig opnamen van het vat gemaakt in twee richtingen loodrecht op elkaar. De berekening van de mate van wernauwing in een slagader wordt aangegeven. Als niet-invasieve methoden van onderzoek werden het OPG-Gee, het periorbitale Doppler onderzoek en het directe Doppler onderzoek met spectraal analyse van het Doppler signaal gebruikt. Bij her OPG-Gee onderzoek werd de uitslag van het onderzoek geinterpreteerd volgens de criteria van Gee, Eikelboom en Machleder. Bij het periorbitale Doppler onderzoek werd de stroomrichting en stroomsnelheid in de periorbitale slagaders bepaald. Bii het directe Doppler onderzoek van de halsslagaders werd de breedte van het Doppler spectrum bepaald en de grootte van de frequentie verandering van het ultrageluid. De resultaten van de niet-invasieve methoden van onderzoek werden vergeleken met de resultaten van de contrast angiografie. Het hoofdstuk besluit met een beschrijving van de wijze waarop de verkregen gegevens werden geanalyseerd en bewerkt.

In hoofdstuk 6 worden de resultaten van de studie besproken. Enkele karakteristieken van de onderzochte groep patienten worden beschreven. De resultaten van het contrast angiografisch onderzoek worden beschreven naar plaats en zijde van het stenotische letsel en de ernst wan deze letsels. Omdat de indirecte, nietinvasieve methoden van onderzoek een indruk geven over een uitgebreider traject van de vaatboom wordt voor deze indirecte methoden van onderzoek het begrip 'arterieel segment' geintroduceerd. Dit 'arteriele segment' is het traject vanaf de aortaboog tor de arteria ophthalmica. Als grad van vernauwing ter vergelijking met de indirecte methoden van onderzoek wordt de ernstigste stenose in dit traject beschouwd. Vervolgens worden de resultaten wan het OPG-Gee, het periorbitale Doppler onderzoek en het directe Doppler onderzoek met spectraal analyse wan het Doppler signaal besproken. Aansluitend op de beschrijving van de resultaten volgt een commentaar en worden de verkregen resultaten vergeleken met resultaten uit andere studies. Met name aan de resultaten van de verschillende niet-invasieve onderzoeken voor het opsporen van stenosen in de arteria carotis interna wordt aandacht besteed. Het blijkt dat het OPG-Gee onderzoek bij de interpretatie van het onderzoek volgens de criteria van Gee, Eikelboom of Machleder voor het opsporen van stenosen met een vernauwing van $30 \%$ of meer een sensitiviteit heeft van respectievelijk $57 \%, 47 \%$ en $48 \%$. De specificiteit van het OPG-Gee volgens deze criteria is respectievelijk $87 \%, 98 \%$ en $97 \%$. Het 
perjorbitale Doppler onderzoek heeft bij deze mate van vernauwing een sensitiviteit van $50 \%$ en een specificiteit van $96 \%$. Het directe Doppler onderzoek blijkt bij vernauwingen wan $30 \%$ of meer een sensitiviteir te hebben van $98 \%$ en een specificiteit $93 \%$. Als de drempel die onderscheid makt tussen 'gezond' en "ziek" wordt verhoogd tot $70 \%$ vernauwing is de sensitiviteit van her OPG-Gee onderzoek volgens de criteria van Gee, Eikelboom en Machleder respectievelijk $76 \%, 69 \%$ en $71 \%$. De specificiteit van het onderzoek is dan respectievelijk $83 \%$, $95 \%$ en $94 \%$. Voor het periorbitale Doppler onderzoek zijn de sensitiviteit en specificiteit onder deze omstandigheden respectievelijk $69 \%$ en $92 \%$. Het directe Doppler onderzoek blijkt dan een sensitiviteit en specificiteit te hebben van respectievelijk $100 \%$ en $75 \%$. Het blijkt dat niet-invasief onderzoek van de extracraniele circulatie betrouwbaar is in het ontdekken van stenosen in de bifurcatie van de arteria carotis. Zelfs stenosen met een geringe graad van diameter reductie kunnen worden ontdekt. Het directe niet-invasieve onderzoek is betrouwbaarder in de opsporing van deze stenosen dan de indirecte methoden van onderzoek. Hiermede wordt geen afbreuk gedaan aan de warde van de indirecte onderzoeken voor het beoordelen van de hemodynamische consequenties van een vernauwing. Deze indirecte methoden kunnen dan ook worden gezien als aanvullend op het directe onderzoek. Het nadeel van het indirecte onderzoek blift echter dat het niet mogelijk is de totale afsluiting van een slagader te onderscheiden van een ernstige vernauwing.

In hoofdstuk 7 wordt een algemene beschouwing over de verrichte onderzoeken gegeven. De onderzoeksmethoden worden vergeleken wat betreft de betrouwbaarheid en de beperkingenen. De in de inleiding gestelde vragen worden beantwoord. De tendens om vernauwingen in de halsslagader op te sporen met behulp van niet-invasieve methoden van onderzoek in plaats van contrast angiografie wordt toegelicht. 


\section{REFERENCES}

1 Adiga KR, Fresso SJ, Nayden J. Noninvasive Methods in the Diagnosis of Extracranial Carond Artery Disease: A Correla* tion with Carotid Arteriography in Eighty Patients.

Angiology 35, 331, 1984 .

2 Alpers BJ, Berry RG, Paddison RM, Anatomical Studies of the Circle of Willis in Normal Brain.

Arch Neurol Psych 81, 409, 1959.

3 Anson BJ, McVay CB,

Surgical Anatomy, wolume 1.

WB Saunders, Philadelphia, 1971.

4 Arbeille Ph, Lapierre F, Benhamou A-C, Salez F, Lagueyrie M, Pourcelot L,

l'Echotomographie et l'analyse spectrale du signal Doppler dans le bilan des lesions carotidiennes.

J Mal Vasic 9, 171, 1984.

5 Archie JP, Faldtman RW,

Critical stenosis of the internal carotid artery.

Surgery $89,67,1981$.

6 Archie JP, Posey PH, Goodson DS,

Accuracy of Digitalized Differential Pulse Timing Oculoplethysmography.

Surg Gynecol Obster 152, 259, 1981.

7 Awad I, Little JR, Modic MT, Furlan A],

Intravenous Digital Subtraction Angiography: An Index of Collateral Cerebral Blood Flow in Intermal Carovid Artery Occlusion.

Stroke 13,469, 1982.

8 Baalen JM van, Jakimowicz JJ, Reneman RS,

Noninvasive Evaluation of Carotid Artery Stenosis - Comparison of Direct and Indirect

Techriques.

Vascular Surgery 18, 88, 1984 .

9 Baalen JM wan, Haeck LBA,

Wardebepaling van de specraalanalyse van het Doppler signaal in de beoordeling van de bifurcatie van de arteria carotis.

Acta Chir Buelg 86, 31, 1986.

10 Baker JD, Barker WF, Machleder HI,

Evaluation of Extracranial Cerebrovascular Disease with Ocular Pneumoplethysmography.

Am $\|$ Surg 136, 206, 1978. 
11 Baker JD, Barker WF, Machleder HI,

Ocular Pneumoplethysmography in the Evaluation of Carotid Stenosis.

Circulation 62, suppl I, 1-1, 1980 .

12 Baker JD, Kaufman M, Machleder $\mathrm{HI}$,

Evaluation of Internal Carotid Stenosis with the Chromopulse, An Automated Ocular Pulse Arrival 'Time Recorder.

Am J Surg 142,212,1981.

13 Bakker RB, Odijk EJA van, Oosterhuis JA,

Binocular fundus reflectometry and ophthamodynamometry in carotid occlusion.

Neurology 29, $108,1979$.

14 Bandyk DF, Levine AW, Pohl L, Tiowne JB,

Classification of Carotid Bifurcation Disease Using Quantitative Doppler Spectrum Analysis.

Arch Surg $120,306,1985$.

15 Barnes RW, Bone GE, Reinertson J, Slaymaker EE, Hokanson DE, Strandness DE Jr, Noninvasive ultrasonic carotid angiography: Prospective validation by conrast angiography.

Surgery $80,328,1976$.

16 Barnes RW, Russell HE, Bone GE, Slaymaker EE,

Doppler Cerebrovascular Examination: Improved Results with Refinements in Technique.

Stroke \&, 468, 1979.

17 Barnes RW,

Hemodynamics for the Vascular Surgeon.

Arch Surg 115, 216, 1980.

18 Barnes $\mathrm{RW}$, Nix L, Rittgers SE,

Audible Interpretation of Carotid Doppler Signals. An Improwed Technique to Define Carotid Artery Disease.

Arch Surg 116, 1185, 1981.

19 Barnes RW, Rittgers SE, Putney WW,

Real-time Doppler Spectrum Analysis.

Arch Surg 117, 52, 1982.

20 Barnett HJM,

Pathogenesis of Transient Ischemic Attacks.

In: Cerebrovascular Diseases.

ed. Schenberg P, Raven Press, New York, 1976.

21 Barnett HJM,

Progress towards stroke prevention: Robert Wartenberg Lecture.

Neurology $30,1212,1980$.

22 Bartynski WS, Darbouze P, Nemir P Jr,

Significance of Ulcerated Plaque in Transient Cerebral Ischemia.

Am J Surg 141, 353, 1981 .

23 Baskett JJ, Beasley MG, Murphy GJ, Hyams DE, Gosling RG, Screening for carotid junction disease by spectral analys is of Doppler signals.

Cardiov Res 11, 147, 1977.

24 Bendick PJ, Glover $\rfloor L$,

Detection of subcritical stenoses by Doppler spectum analysis.

Surgery $91,707,1982$. 
25 Bergh $\mathrm{R}$ van de,

De subcorticale angioarchitectur van het menselik telencephalon.

Arscia Uitgaven, Brussel, 1960.

26 Berguer $\mathbb{R}$, Hwang $\mathbb{N H C}$,

Critical Arterial Stenosis: A Theoretical and Experimental Solution.

Ann Surg 180, 39, 1974 .

27 Berkowitz HD,

Diagnostic Accuracy of Ocular Pneumoplethysmography Attachment for Pulse Volume

Recorder.

Arch Surg 115, 190, 1980 .

28 Bernstein EF,

Additional Noninvasive Techniques for Cerebrowascular Diagnosis.

In: Intraduction to Vascular Ultrasonography.

ed. Zwiebel WJ, Grune \& Stratton, New York, 1982.

29 Blackshear WM Jr, Phillips DJ, Thiele BL. Hirsch JH, Chikos PM, Marinelli MR, Ward $K J$, Strandness DE Jr,

Detection of carotid occlusive disease by ultrasonic imaging and pulsed Doppler spectrum analysis.

Surgery $86,698,1979$.

30 Blackshear WM Jr, Thicle BL, Harley JD, Chikos PM, Strandness DE Jr,

A Prospective Evaluation of Oculoplethysmography and Carotid Phonoangiography.

Surg Gynecol Obstet 148,201, 1979.

31 Blackshear WM Jr, Phillips DJ, Chikos PM, Harley JD, Thiele BL, Strandness DE Jr, Carotid Artery Velocity Patterns in Normal and Stenotic Vessels.

Stroke 11, 67, 1980 .

32 Blaisdell FW, Hall AD, Thomas AN, Ros: SI,

Cerebrovascular Occlusive Disaese. Experience with Panarceriography in 300 Consecutive

Cases.

Calif Medic 103, 321, 1965.

33 Blaisdell FW, Glickman M, Trunkey DD,

Ulcerated arheroma of the carotid artery.

Arch Surg 108, 491, 1974.

34 Boer J de,

Stroming in slagaderen. Beknopt overzicht wan de fysica, de fysiologie en de partrologie.

Bohn, Seheltema en Holkema, Utrecht, 1978.

35 Bogousslavsky $\mathbb{J}$, Hachinski VC, Boughner DR, fox AJ, Vinuela F, Barnett $H J M$,

Cardiac and Arterial Lesions in Carotid Transient Ischemic Autacks.

Arcin Naurol 43,223, 1986.

36 Bone GE, Barnes RW,

Limitations of the Doppler cerebrovascular examination in hemispheric cerebral ischemia.

Surgery $79,577,1976$.

37 Bone GE, Dickinson D, Pomajzl MJ,

A prospective evaluation of indirect methods for detecting carotid atherosclerosis.

Surg Gynecol Obstet 152, 587, 1981 .

\section{Bosniak MA,}

An analysis of some anatomic-roentgenologic aspects of the brachiocephalic vessels.

An \Roengenol $91,1222,1964$. 
39 Brener $B J$, Raines $\mathbb{J K}$, Darling $\mathbb{R C}$, Austen $\mathrm{G}$,

Measurement of Systolic Femoral Arteriall Pressure during Reactive Hyperenia.

Circulation 49-50, suppl II, II-259, 1974.

40 Breslau PJ,

Ultrasonic Duplex Scanning in the evaluation of Carotid Artery Disease.

Thesis, Maastricht, The Netherlands, 1981.

41 Breslau PJ, Knox RA, Beach KW, Thiele BL, Philles DJ, Chikos PM, Strandness DE

Jr,

Ultrasonic duplex scanning with spectral analysis in extracranial carocid artery disease.

Comparison with contrast arteriography.

Vasc Diagn Ther $17,1982$.

42 Brice $J G$, Dowsett DJ, Lowe RD,

Hemodynamic Effects of Carotid Artery Stenosis.

Brit Med J 2, 1363, 1964.

43 Brockenbrough EC, Lawrence C, Schwenk WG,

Ocular plethysmography: a new technique for the evaluation of carotid obstructive disease.

Rev Surg 24, 299, 1967 .

44 Brockenbrough $\mathbb{E C}$,

Screening for the Prevention of Stroke: Use of a Dopplen Flowmeter.

Information and Education Resource Support Unir W/ashington / Alaska Regional Medical Program, 1969.

45 Brockenbrough $\mathbb{E} C$,

Periorbital Doppler Velocity Evaluation of Carotid Obstruction.

In: Noninvasive Diagnosric Techniques in Vascular Disease.

ed. Bernstein EF, The CV Mosby Company, Saint Louis, 1978.

46. Brown PM, Johnston $\mathrm{KW}$,

The difficulty of quantifying the severity of carotid stenosis.

Surgery $92,468,1982$.

47 Brown PM, Johnston KW, Douville Y,

Detection of occlusive disease of the carotid artery with continuous wave Doppler spectral analysis.

Surg Gynecoll Obstet 155, 183, 1982.

48 Brown PM, Johnston KW, Kassann M, Cobbold RSC,

A critical study of ultrasound spectral analysis for dececring carotid disease.

Ultrasound in Med \& Biol 8, 515, 1982.

49 Bruins Slot H, Strijbosch L, Greep JM,

Interobserver Variability in Single-plane Aortography.

Surgery $90,497,1981$.

50 Budingen $\mathrm{HJ}$, Reutern $\mathrm{G}-\mathrm{M}$ von, Freund $\mathrm{H}-\mathrm{J}$,

Doppler-Sonographie der extrakraniellen Hirnarterien.

Georg Thieme Verlag, Stuttgart, 1982.

51 Burger RD, Barnes RW,

Choice of Ophthandic Artery Branch for Doppler Cerebrowascular Examination: Advan tages of Frontal Artery.

Angiology $28,421,1977$.

52 Burton AC,

Physiology and Biophysics of the Circulation, 2 nd ed.

Year Book Medical Publishers, Chicago, 1972. 
53 Carter $\mathrm{SA}$,

Response of Ankle Systolic Pressure to Leg Excencise in Mild or Questonable Arterial Disease.

NEng J Med 287, 578, 1972 .

54 Chikos PM, Fisher LD, Hirsch $\int H$, Harley $\int D$. Thiele BL, Surandness DE Jr,

Observer Varabihy in Evaluatung Extractanial Carotid Artery Stenosis.

Stroke 14,885, 1983.

55 Coghlan BA, Taylor MG, King DH,

On-line Display of Doppler-shif Spectra by a New Time Compression Analyser.

In: Cardiovascular Applications of Doppler Ultrasound.

ed. Reneman RS, North Holland Publishing Company, Amsterdam, 1974.

56 Comerota AJ, Cranley J], Cook SE,

Real-time B-mode carotid imaging in diagnosis of cerebrovascular disease.

Surgery 89, 718, 1981.

57 Croft RJ, Eעlam LD, Harrison $M \| J G_{3}$

The Accuracy of Carotid Angiography in Determining Canotid Artery Disease.

In: Arteriopathies Cerebrales Extracraniennes Asymptomariques.

ed. Courbier $\mathbb{R}$, Documentation Medicale Oberval, Lyon, 1980.

58 Croft RJ, Ellam L.D, Harrison M\|G,

Accuracy of Carotid Angiography in the Assessment of Atheroma of the Internal Carotid Artery.

Lancet 2, $997,1980$.

59 Cross G, Light LG,

Non-invasive Intrathoracic Blood Velocity Measurement in the Assessment of Cardiovas cular Function.

J Biomed Eng 9, 464, 1974.

60 Curry GR, White DN,

Color Coded Ultrasonic Differential Velocity Arterial Scanner (Echoflow)

Ulrasound in Med \& Biol 4, 27, 1978.

61 Davis HH, Siegel BA, Sherman LA, Heanon WA, Naidich TP, Joist $\ H$, Welch M.J, Scintigraphic Detection of Carotid Atherosclerosis with In-111-labeled Autologous Platelecs.

Circulation 61, 982, 1980.

62 DeWeese JA, May AG, Lipchik EO, Rob CG,

Anatomic and Hemodynamic Correlations in Carotid Artery Stenosis.

Stroke 1, $149,1970$.

63 Dongen MEH van, Steenhoven AA van,

Some Fluid Dynamical Aspects of: Arterial Flow.

In: Doppler Ultrasound in the Diagnosis of Cerebrovascular Discase.

eds. Reneman RS, Hoeks APG, Research Studies Press, Chichester, 1982.

64 Douville $Y$, Johuston KW, Kassam M,

Determination of the hemody namic factors which influence the carotid Doppler spectral broadening.

Ultrasound in Med \& Biol $₫ 1,417,1985$.

65 Duke LJ, Slaymaker EE, Lamberth WC, Wright CB,

Results of Ophthalmosonomery and Supraorbital Photoplerhysmography in Evaluaring Carotid Arterial Stenoses.

Circulation 60, suppl 1, 1-127, 1979. 
66 Duncan GW, Gruber JO, Dewey CF, Myers GS, Lees RS,

Evaluation of carotid stenosis by phonoangiography.

N Eng I Med 293, 1124, 1975.

67 Dyken ML,

Evaluacion of Cerebral Ischemia: Transient, Focal, Lateralizing, and Nonhemispheric Ischemic Artacks.

In: Cerebrovascular Insufficiency.

eds. Bergan JJ, Yao JST, Grune \& Stration, New York, 1983.

68 Earnest F [V, Houser W, Forbes GS, Kispert DB, Folger WN, Sundt TM Jr, The Accuracy and Limitations of Intravenous Digital Subtraction Angiography in the Evaluation of Atherosclerotic Cerebrovascular Disease: Angiographic and Surgical Correlation.

Mayo Clin Proc 58, 735, 1983.

69 Eecken $H$ van der,

Discussion of: "Collateral Circulation of the Brain"

Neurology $11,16,1961$.

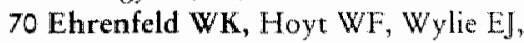

Embolization and Transient Blindness from Carotid Atheroma Surgical Considerations. Arch Surg 93, 787, 1966.

71 Eikelboom BC, Vermeulen FEE,

The Value of Ocular Pneumoplethysmography versus Angiography in the Determination of the Potential Collateral Hemispheric Circulation.

In: Noninvasive Cardiovascular Diagnosis, second edition,

ed. Dietrich EB, PSG Publishing Company, Littleton, 1980.

72 Eikelboom $\mathrm{BC}$,

Evaluation of Carotid Artery Disease and Potential Collateral Circularion by Ocular Pneumoplechysmograptiy.

Thesis, Utrecht, The Netherland, 1981.

73 Eikelboom BC, Ackerstaff RGA, Moll FL, Taks ACJM, Vermeulen FEE,

Is Carotid Coiling Hemodynamically Significant?

In: Noninvasive Assessment of the Cardiovascular System, Diagnostic Principles and Tiechniques.

ed. Diethrich EB, John Wright, Boston, 198.2.

74 Eikelboom BC, Riles TS, Folcarelli P, Imparato AM,

Griteria for Interpretacion of Ocular Pneumoplethysmography (Gee).

Arch Surg 118, 1169, 1983.

75 Eikelboom BC, Riles TR, Mintzer R, Baumann FG, DeFillip G, Lin J. Imparato AM, Inaccuracy of Angiography in the Diagnosis of Carotid Ulceration.

Stroke 14, 882, 1983 .

76 Eisenberg RL, Nemzek WR, Moore WS, Mani RL,

Relationship of Transient Ischemic Aracks and Angiographically Demonstrable Lesions of Carotid Artery.

Stroke 8, 483, 1977.

77 Fell G, Breslau PJ, Knox RA, Phillips DJ, Thiele BL, Strandness DE Jr, Importance of noninvasive ultrasonic-Doppler testing in the evaluation of patients with asymptomatic carotid bruits.

Am Heart I 102, 221, 1981 . 
78 Fell G, Phillps DJ, Chkos PM, Harley JD, Thiele BL, Strandness DE Ir, Ultrasonic Duplex Scanning for Disease of the Carowd Artery.

Circulation 64, 1191, 1981.

79 Fischer $G G$, Anderson DC, Farber $\mathbb{R}$, Lebow $\$$

Prediction of Carotid Disease by Ularasound and Digitall Subraction Angiography.

Arch Weurol 42, 224, 1985 .

80 Fischer $M$, Alewander $K$, Vogelsang $H$,

Erfahrungen mit der Ditectional-Doppler-Ultraschall-Sonographie der A. supratrochlearis bei Verschlussprozessen der A. caroris communis.

VASA $8,289,1979$

81 Fischer $M$, Alexander $K$, Haller $P$, Vogelsang $H$,

Fluoreszenz-Angiographie der Supraorbitalregion. Fune nicht belastende Untersuchung zur Diagnose von Verschlussprozessen der Arteria caroris.

VASA 9,266, 1980 .

82 Fischer M, Allexander K, Vogelsang H, Haller P,

Diagnosuk der Verschlussprozessen der Arteria carotis mit der supraorbitalen Lichtpllethysmographie.

VASA 9, 15, 1980.

83 Fischer M, Alexander $\mathbb{K}$,

Reproducibility of Carotid Artery Doppler Frequency Measurements.

Stroke 16, 973, 1985.

84 Fisher CM,

Obserwations of the fundus oculi in transient monocular blindness.

Neurology 9,333, 1959 .

85 Franklin DL, Schlegel W, Rushmer RF,

Blood Flow Measured by Doppler Frequency Shift of Backscattered Ultrasound.

Science $\mathbb{1 3 4}, 564,1961$.

86 Galin MA, Baras 1, Ciavero R, Best M.

Compression and Suction Ophthalmodynamometry.

Am J Ophthalmol 67, 388, 1969 .

87 Galin MA, Baras I, Best M, Cavero R, Friedman B,

Methods of Sucrion Ophthalmodynamometry.

Ann Ophrhal2, 439, 1970.

88 Gee W, Snith CA, Hinson CE, Wylie EJ,

Ocular Preumoplethysmography in Carotid Artery Disease.

Med Instrum 8, 244, 1974.

89 Gee W, Matigan JT, Wylle EJ,

Mcasurement of Collateral Cerebral Hemispheric Blood Pressure by Ocular Pneumoplethysmography.

An J Surg $130,121,1975$.

90 Gee W, Oller DW, Wylie E],

Nonimvasive Diagnosis of Carotid Oeclusion by Ocular Pneumoplethysmography.

Stroke 7, 18, 1976 .

91 Gee W, Oller DW, Amundsen DG, Goodreau JI,

The Asympromatic Carotid Bruit and the Ocular Pneumoplethysmography.

Arch Surg $112,1381,1977$. 
92 Gee W, Kaupp HA, McDonald KM, Lin FZ, Curry IL,

Spontaneous Dissecrion of Internal Carotid Arteries. Spontaneous Resolution Documented by Serial Ocular Pneumoplechysmography and Angiography.

Arch Surg 115, 944, 1980 .

93 Gee W, McDonald KM, Kaupp HA, Celani JS, Bast RG,

Carotid Stenosis Plus Occlusion: Endarterectomy or Bypass?

Arch Surg 115, 183, 1980 .

94 Gee W,

Carotid Physiology with Ocular Pneumoplethysmography.

Stroke 13,666, 1982.

95 Gee W,

Ocular Pneumoplethysmography (OPG-Gee).

In: Practical Noninvasive Vascular Diagnosis.

eds. Kempczinski RE, Yao JST, Year Book Medical Publishers, Chicago, 1982.

96 Genton E, Barnett HJM, Fields WS, Gent M, Hoak JC, XIV.

Cerebral Ischemia: The Role of Thrombosis and of Antithrombotic Therapy. Seudy Group on Antithrombotic Therapy.

Stroke 8, 150, 1977.

97 Ginsberg MD, Greenwood SA, Goldberg HI,

Limitations of Quantitative Oculoplethysmography and of Directional Doppler Ulurasonography in Cerebrowascular Diagnosis: Assessment of an Mir-filled OPG System.

Stroke 12, 27, 1981.

98 Glower JL, Bendick PJ, Jackson VP, Becker GJ, Dilley RS, Holden RW,

Duplex Ultrasonography, Digital Subtraction Angiography, and Conventional Angiography in Assessing Carotid Atheroscterosis.

Arch Surg 119, 664, 1984.

99 Goldman M, Leung JO, Aukland A, Hawker RJ, Drole Z, McCollum CN,

111-Indium Platelet Imaging, Doppler Spectral Analysis and Angiography Compared in Patients with Transient Cerebral Ischemia.

Stroke 14, 752, 1983.

100 Grady PA,

Pathophysiology of Extracranial Cerebral Arterial Stenosis - A Critical Review.

Stroke $15,224,1984$.

101 Gross WS, Verta M I, Van Bellen B, Bergan JJ, Yao JST,

Comparison of nominvasive diagnostic techniques in carotid artery occlusive disease.

Surgery $82,271,1977$.

102 Grotta J. Ackerman R, Correia J, Fallick $G$, Chang. J.

Whole Blood Viscosity Parameters and Cerebral Blood Flow.

Stroke 13, 296, 1982.

103 Hardesty WH, Roberts B, Toole JF, Royster HP,

Studies of Carotid Artery Blood Flow in Man.

N Eng J Med 263, 944, 1960.

104 Harrison MIG, Masshall I,

Angiographic appearance of carotid bifurcation in patients with compleced stroke, transient ischaemic attacks, and cerebral rumour.

Brit Med J 1, 205, 1976. 
105 Harrison M $\mathrm{G}$, Marshall].

The linding of thrombus at carotid endarterectomy and its retation to the timing of surgery.

Br \Surg 64, 511, 1977 .

106 Hass WK, Wields WS, North RR, Kricheff II, Chase NE, Baner RB,

Joint Study of Extracranial Arterial Ocdusion. Part 11 : Arteriography, "Techniques, Sites, and Complications.

JAMA 203, 961, 1968.

107 Hatle L, Angelsen B,

Doppler Ultrasound in Cardiology, Physical Principles and Clinical Applications, chapter

2. Physics of Blood Flow.

Lea \& Febiger, Philadelphia, 1982.

108 Hawkins $T D$,

"The collateral anastomoses in cerebro-vascular occlusion.

Clin Radiol 17, 203, 1966 .

109 Hennerici $M$, Aulich A, Sandmann W, Freund HJ,

Incidence of Asymptomatic Extracranial Arterial Disease.

Sroke 12,750, 1981 .

110 Hennerici M, Reifschneider $G$, Trockel U, Aulich A,

Detection of Early Atherosclerotic Lesions by Duplex Scaming of the Carotid Artery.

$\mathrm{J}$ Clin Ultrasound $12,455,1984$.

111 Hertzer NR, Santoscoy TG, Langston RHS,

Accuracy of carotid compression tonography in the diagnosis of carotid artery stenosis.

Correlation wich arteriography in 300 patients.

Cieveland Clinic Quarterly 47,79, 1980.

112 Hessel SJ, Adams DF, Abrams HL,

Complications of Angiography.

Radiology 138, 273, 1981.

113 Hewitt RL, Schramel RJ, Pearce CW, Weichert RF:

Cerebral Arterial Insufficiency, Angiography and Surgical Treatment.

Am J Surg 120,334, 1970.

114 Hobson RW, Berry SM, Jamil Z, Mehta K, Hart L, Simpson H,

Oculoplethysmography and Pulsed Doppler Ultrasonic Imaging in Diagnosis of Carotid Arterial Disease.

Surg Gynecol Obstet 152, 433,1981.

115 Hoeks APG, Reneman RS, Ruissen CJ, Smeets FAM,

Possibilities and Limitations of Pulsed Doppler Systems.

In: Echocardiology.

ed. Lance $\mathrm{CI}^{4}$. Martinus Nijhof, The Hague, 1979.

116 Hoeks APG,

On the development of a multi-gate pulsed Doppler system with serial data-processing. "Thesis, Maastricht, The Netherlands, 1982.

117 Hoeks APG, Peeters HHPM, Ruissen CJ, Reneman RS,

A Novel Frequency Estimator for Sampled Doppler Signals.

IEEE Transactions Bion $\operatorname{Eng} 31,212, \mathbb{1 9 8 4}$.

118 Hofferberth B, Gotschald M, Dykan S,

Comparison of Doppler Sonography and Plate Thermography for Detection of Carotid

Artery Stenosis.

Stroke 11, 27, 1980. 
119 Hollenhorst RW,

Significance of Bright Plaques in the Recinal Arterioles.

JAMA $178,23,1961$.

120 Honig $C R$,

Modern Cardiovascular Physiology, chapter 8.

Little, Brown and Company, Boston, 1981.

121 Huckman MS, Sherit. GI, Neems RL, Tinor ' $\mathrm{T}$,

Transfemoral Cerebral Arteriography versus Direct Percutaneous Caronid and Brachial Arteriography: A Comparison of Complication Rates.

Radiology $132,93,1979$.

122 Ishikawa $S$, Handa J, Meyer JS, Huber P,

Haemodynamics of the circle of Willis and the leptomeningeat anastomoses: an elecrom magneric flowmeter study of intracranial arrerial ocdusion in the monkey.

J Neurol Neurosurg Psychat, 28, $124,1965$.

123 Javid $\mathrm{H}_{*}$

Development of Carotid Plaque.

Am J Surg 138, 224, 1979 .

124 Johnston KW, Maruzzo BC, Cobbold RSC,

Doppler Methods for Quantitative Measurement and Localizacion of Peripheral Arcerial

Occlusive Disease by Analysis of the Blood Flow Velocity Wavetorm.

Ultrasound in Med \& Biol 4, 209, 1978.

125 Johnston $\mathrm{KW}$, Brown PM, Kassam $\mathrm{M}$,

Problems of Carotid Doppler Scanning Which Can Be Overcome by Using Frequeney

Analysis.

Stroke 13, 660, 1982.

126 Johnston KW, Haynes RB, Douville $\mathrm{Y}$, Lally ME, Brown PM, Cobbold RSC,

Accuracy of carotid Doppler peak frequency analysis: results determined by receiver operating characteristic curves and likelihood ratios.

J Vasc Surg 2, 515, 1985.

127 Jones AM, Biller J, Cowley AR, Howard G, McKinney WM, Toole $\| F$,

Extracramal Carotid Artery Arreriosclerosis. Diagnosis with Continuous-W/ave Doppler and Real-time Ultrasound Studies.

Arch Neurol 39, 393, 1982.

128 Jonkman EJ, Mosmans PCM,

Basic Anatomy, Physiology and Pathology of Human Cerebral Circulation.

In: Doppler Ultrasound in the Diagnosis of Cetebrovascular Disense.

eds. Reneman RS, Hoeks APG, Research Sudies Press, Chichester, 1982.

129 Kadota $E$, Kaneda $H$, Makinaga $G$, Taneda $M$, Irino 'T",

Pattern Difference of Reversed Ophthalmic Blood Filow Between Occlusion and Stenosis of the Internal Carotid Artery. An Ultrasonic Doppler Study.

Stroke $13,381,1982$.

130 Kalman $P G$, Johnston KW, Zuech P, Kassam M, Poots $\mathbb{K}_{\text {. }}$

In vitro comparison of alternative me hods for quantifying the severity of Doppler spectral broadening for the diagnosis of carotid arterial occlusive disease.

Ultrasound in Med \&. Biol 11, 435, 1985.

131 Kaplan HA,

Colateral Circulation of the Brain.

Neurology $11,9,1961$. 
132 Kapsch D, Cook L, Lichi E, Silver D,

Use of Combined Oculoplethysmograpty, Carocid Phonoangiography and Doppler in the Non-Invasive Diagnosis of Extracranial Carotid Occlusive Disease.

Stroke 12,317, 1981.

133 Kartchner MM, Mckac LP,

Auscultation for Carotid Brwis in Cerebrovascular Insufficiency.

JAMA $210,494,1969$.

134 Kartchner MM, McRae LP, Morrison FD,

Noninvasive Detection and Evaluation of Carotid Occlusive Disaese.

Arch Surg 106, 528, 1973 .

135 Kartchner MM, McRae LP, Crain V, Whitaker B,

Oculoplethysmography: An Adjunct to Arteriography in the Diagnosis of Extracranial Carotid Occlusive Disease.

AmJ Surg 132, 728, 1976.

136 Kartchner MM, McRae LD,

Noninvasive evaluation and management of the "asymptomatic" carotid bruit.

Surgery $82,840,1977$.

137 Kassam M, Johnston KW, Cobbold RSC,

Quantitative estimation of spectral broadening for the diagnosis of carotid arterial disaese: method and in vitro results.

Ulurasound in Med \& Biol 11, 425, 1985.

138 Katz DM, Smith RA, Otis SM, Dalessio DJ,

Doppler Sonography Diagnosis of Cerebrovascular Discase.

Stroke 7.,439, 1976.

139 Keagy BA, Pharr WF, "Thomas DD, Browes DE,

Oculoplethysmography/Carotid Phonoangiography. Its Value as a Screening Test in Patients With Suspected Carotid Artery Stenosis.

Arch Surg 115, 1199, 1980.

140 Keagy BA, Pharr WF, Thomas D, Bowes DE,

Comparison of Oculoplethysmography/Carotid Phonoangiography with Duplex Scant Spectral Analysis in the Detection of Carotid Artery Stenosis.

Stroke $13,43,1982$.

141 Keitzer WF, Fry WJ, Kraft RO, DeWeese MS, Sloane HE,

Hemodynamic mechanism for pulse changes seen in occlusive vascular disease.

Surgery $57,163,1965$.

142 Keller HM, Meier WE, Anliker M, Kumpe DA,

Nominvasive Measurement of Velocity Profiles and Blood Flow in the Common Carocid Artery by Pulsed Doppler Ulerasonnd.

Stroke $7,370,1976$.

\section{Kempczinskil RF,}

A combined approach to the noninvasive diagnosis at carotid artery occlusive disease.

Surgery $85,689,1979$.

144 Kendell RE, Marshall J,

Role of Hypotension in the Genesis of Transient Focal Cerebral Ischaenic Atcacks.

Bric Med J 2, 344, 1963.

145 Kind $\mathrm{GW}$, Youmans $\int R$,

The Effect of Stricture Length on Critical Arterial Stenosis.

Surg Gynecol Obsten $128,729,1969$. 
146 Kistler JP, Les RS, Friedman J, Pessin M, Moh JP, Robertson GS, Ojemama RG, The Bruit of Carotid Stenosis versus Radiated Basal Hearr Murmurs. Differentiation by Phonoangiography.

Circulation $57,975,1978$.

147 Kistler JP, Lees RS, Miller A, Crowell RM, Roberson G,

Correlation of Spectral Phonoangiography and Carocid Angiography with Gross Pachology in Carotid Stenosis.

N Eng J Med 305, 417, 1981 .

148 Knox RA, Bresiau $\mathbb{P}$ J, Strandness DE Jr,

Quantitative Carotid Phonoangiography.

Stroke 12,798, 1981.

149 Knox RA, Breslau [J, Strandness DF Jr,

A simple parameter for accurate detection of severe carotid disease.

Br J Surg 69, 230, 1982.

150 Knox RA, Greene FM, Beach K, Phillips DJ, Chikos PM, Strandness DE Jr, Compter Rased Classification of Carotid Arterial Disease: A Prospective Assessment. Stroke 13,589, 1982.

151 Kollarits CR, Lubow M, Hissong $\mathrm{SL}$,

Retinal Strokes, I: Incidence of Carotid A theromata.

JAMA 222, 1273, 1972.

\section{Koran LM,}

The reliability of clinical methods, data and judgements.

N Eng 』Med 642, 1975.

153 Khramer SP,

On Function of Circle Of Willis.

J Exp Med 15, 348, 1912.

154 Krause $\mathrm{H}$, Segard M, Carey $\mathbb{P}$, Bernstein EF, Fronek A,

Doppler Power Frequency Spectrum Analysis in the Diagnosis of Carond Artery Disease. Stroke 1.5, 351, 1984.

155 Ku DN, Giddens DP,

Pulsatile Flow in a Model Carotid Bifurcation.

Arteriosclarosis 3, 31, 1983 .

\section{Kuhn RA,}

Succcesstul Radiographic Denonstration of the Human Circle of Willis. JAMA $175,769,1961$.

157 Larson JP Jr,

Anaesthesia and Concrol of the Cerebral Circulation.

In: Extracranial Cerebrovascular Disease: Diagnosis and Management. eds. Wylle EJ, Ehrenfeld WK, WB Sanders, Philadehphia, 1970.

158 Lavy S, Stern S, Melamed E, Cooper G, Keren A, Levy P, Effect of Chronic Atrial Fibrillation on Regional Cerebral Blood Flow.

Stroke 11,35, 1980.

159 Lazorthes $G$, Gowaze $A$, Santini Ju, Salamon $G$,

The Arterial Circle of the Brain (circulus arteriosus cerebri).

Anatomia Clinica 1, 241, 1979.

160 Lee BY, Tranor FS, Thoden WR, Kavner D,

Risk Factors in Atherosclerosis.

In: Handbook of Noninvasive Diagnostic Techniques in Vascular Surgery, chapter 8.

Appleton Century Crofts, New York, 1981. 
161 Lee BY, Trainor FS, Thoden WR, Kawner D,

Diagnostic Techniques in Perspective: Visualization, Scanning, Sounding.

In: Handbook of Noninvasive Diagnostic Techniques in Vascular Surgery, chapter 6.

Appleton Century Crofts, New York, 1981.

162 Lees RS, Dewey CF $\int \mathrm{r}$,

Phonoangiography : A New Noninasive Diagnostic Method for Studying Arterial

Disease.

Proc Nat Acad Sci 67, 935, 1970 .

163 Lees RS, Kister JP, Sanders D*

Duplex Dopplet Scanning and Spectral Bruit Analysis for Diagnosing Carotid Stenosis.

Circulation 66, suppl I, 1-102, 1982.

164 Lemak NA, Fields WS,

The Reliability of Clinical Predictors of Extracranial Artery Disease.

Stroke 7, 377, 1976.

165 Lewis RR, Beasley MG, Hyams DE, Gosling RG,

Imaging the Carotid Bifurcation Using Contmuous-Wave Doppler-Shife Ultrasound and Spectral Analysis.

Stroke $9,465,1978$.

166 Lewis RR, Padaychee TS, Gosling $\mathbb{R G}$,

Ulrasound Screening for Internal Carotid Disease-Il. Sensitivicy and Specificity of a Single Sire Periorbital Artery Test.

Ultrasound in Med \& Biol 10,17, 1984.

167 Little JR, Furlan AJ, Modic MT, Weinstein MA,

Digital Subtraction Angiography in Cerebrovascular Disease.

Stroke 13, 557, 1982.

168 LoGerfo FW, Crawshaw HM, Nowak M, Serrallach E, Quist WC, Valeri CR,

Effect of Flow Split on Separation and Stagnation in a Model Vascular Biturcarion.

Srroke 12,660, 1981 .

169 Lusby RJ, Woodcock JP, Skidmore R, Jeans WD, Hope D'T, Baird R,

Carotid Artery Disease: A Prospective Evaluation of Pulsed Doppler Imaging.

Uhrasound in Med \& Biol 7, 365, 1981.

170 Lye CR, Sumner DS, Strandmess DE $\int r$,

The Accuracy of the Supraorbital Doppler Examination in the Diagnosis of Hemodynamically Significant Caronid Occlusive Disease.

Surgery $79,42,1976$.

171 Lynch TG Wright CB, Miller EV,

Oculopnomoplerhysmography, Doppler Examinarion, and Supraorbital Photoplethys mography. A Comparison of Hemodynamic "Techniques in Assessing Cerebrowascular Occlusive Disanse.

Ann Surg 194,731, 1981 .

172 Lynch TG, Wright CB, Miller EV, Slaymaker EE,

Evaluation of Cerebrovascular Doppler Examination and Oculopneumoplethysmography in a Clinical Perspective.

Stroke $12,325,1981$.

173 Lynch TG, Wright CB, Miller EV, Slaymaker EE,

Comparison of Continuous-wave Doppler Imaging, Oculopneumoplethysmography, and the Cerebrovascular Doppler Examination.

Cinculation 66, suppl I, I-106, 1982. 
174 Macpherson PC, Meldrum SJ, Tunstall-Pedoe DS,

Angioscan: a spectum analyser for we with ultrasonic Doppler velocimeters.

J Med Eng Technol 5, 84, 1981 .

175 Malcolm AD, Roach MR,

Flow Disturbances at the Apex and Lateral Angles of a Variety of Bifurcarion Models and Their Role in Development and Manisfestations of Arterial Disease.

Stroke 10, 335, 1979.

176 Malone JM, Bean B, Laguna J, Hamilron R, Labadie E, Moore WS,

Diagnosis of Carotid Artery Stenosis. Comparison of Oculoplethysmography and Dop-

pler Supraorbital Examination.

Ann Surg 191, 347, 1980.

177 Mani RL, Eisenberg RL, McDonald EJ, PollockJA, Mani JR,

Complications of Catheter Cerebral Arteriography: Analysis of 5000 Procedures. Part I:

Criceria and Incidence.

Am J Roentgenol 131, 861, 1978.

178 Maroon JC, Campbell RL, Dyken ML,

Internal Carotid Attery Occlusion Diagnosed by Dopplar Ultrasound.

Stroke 1, 122, 1970.

179 Martinez SA, Oller $\mathrm{DW}$, Gee W, defries $\mathrm{HO}$,

Elective Carotid Artery Resection.

Arch Otolaryngology 101, 744, 1975.

180 May AG, DeWeese JA, Rob CG,

Hemodynamic effects of arteral stenosis.

Surgery $53,513,1963$.

181 MeCreary JA, Schellhas KP, Brant-Zawadzki M, Norman D, Newton TH, Outpatient DSA in Cerebrovascular Disaese Using Transbrachial Arch Injections.

Am J Roentgenol 145, 941, 1985.

182 McDonald DA, Potter JM,

The Distribution of Blood to the Brain.

J Physiol 114, 356, 1951.

183 McDonald DA,

Blood Flow in Arteries.

Williams and Wilkins, Baltumore, 1960.

184 McDonald KM, Gee W, Kaupp HA, Bast RG,

Screening For Significant Carotid Stenosis by Ocular Pncumoplethysmogmaphy.

An J Surg 137, 244, 1979.

185 McDonald PT, Rich NM, Collins GI, Andersen CA, Kozlofl L,

Doppler Cerebrovascular Examination, Oculoplethy smography, and Ocular Pneumoplethysmography. Use in Detection of Carotid Disease: A Prospective Clinical Sudy.

Arch Surg 113, 1341, 1978.

186 McDonald PT, Rich NM, Collins GJ, Kozloff L. Andersen CA,

Ocular Pneumoplethysmography: Detection of Carotid Occlusive Disitese.

Ann Surg $189,44,1979$.

187 McNeil BJ, Keeler E, Adelstein SJ,

Primer on certain elements of medical decision making.

$N$ Eng $₫$ Med 293,211, 1975. 
188 McRae $\mathbb{L}$, Kartcher MM,

Oculoplechysmography: Timed Comparison of Ocular Pulses and Carotid Phonoangiography.

In: Noninvasive Diagnostic Techniques in Vascular Disease.

ed. Bernstein EF, The CV Mosby Company, Sant Lowis, 1978.

189 McRae LP, Kartchner MM,

Carotid Phonoangiography.

In: Practical Noninwasive Vascular Diagnosis.

eds. Kempczinski RE, Yao JST, Year Book Medical Publishers, Chicago, 1982.

190 McRae LP, Kartcher MM,

Oculloplethysmography.

In: Practical Noninvasive Vascular Diagnosis.

eds. Kempczinski RF, Yao JST, Year Book Medical Publishers, Chicago, 1982.

191 Mellamed E, Lavy S, Bentin S, Cooper $G$, Rinot $Y$,

Reduction in Regional Cerebral Blood Flow During Nomal Aging in Man.

Stroke 11, 31, 1980.

192 Mercier LA, Greenleaf JF, Evans TC Jr, Sandok BA, Hattery RR,

High-Resolution Ultrasound Arteriography: A Comparison With Carotid Angiography.

In: Noninyasive Diagnostic "Techniques in Vascular Disease.

ed. Bernstein EF, "The CV Mosby Company, Saint Louis, 1978.

193 Merode 'T' van, Hick P, Hoeks APG, Reneman RS,

Limitations of Doppler Spectral Broadening in the Early Detection of Carotid Artery

Discase Due to the Size of the Sample Volume.

Ultrasound in Med \& Biol 9, 581, 1983.

194 Merode T van, Hick. P, Hoeks APG, Reneman RS,

Serum HDL/Total Cholesterol Ratio and Blood Pressure in Asymptomatic Atherosclerotic Lesions of the Cervical Carotid Arteries in Men.

Stroke 16, 34, 1985.

195 Mettinger KL, Larsson S, Ericson K, Casseborn S,

Detection of atheroscterotic plaques in carotid arteries by the use of 123 -I-fibrinogen.

Lancet 242, 1978.

196. Metz H, Murray-Leslie RM, Bannister RG, Bull JWD, Marshall J,

Kinking of the Internal Carotid Artery in Relation to Cerebrovascular Disease.

Lancet $1,424,196 \|$.

197 Miller A, Lees RS, Kistler JP, Abbott WM.

Spectral Analysis of Arterial Bruits (Phonoangiography): Experimental Validation.

Circulation 61, 515, 1980 .

198 Mol JMFA,

Doppler-haematotachografisch onderzoek buj cerebrale circulatiestoomissen.

Thesis, Utrecht, The Netherlands, 1973.

199. Mol JMFA,

The Clinical Use of Doppler Hematographic Investigation in Cerebral Circulation Disurbances.

In: Doppler Ultrasound in the Diagnosis of Cerebrovascular Disace.

eds. Reneman RS, Hoeks APG, Research Studies Press, Chichester, 1982.

200 Moniz E, Lima A, De Lacerda R,

Hemiplegies par thrombose de la carotide interne.

Presse Med 45, 977, 1937. 
201 Moore WS, Bean B, Burron $R$, Goldstone J,

The use of ophthamosonomery in the diagnosis of carotid arrery srenosis

Surgery 82, 107, 1977 .

202 Moore WS, Boren C, Malone JM, Roon AJ, Eisenberg R, Goldstone J, Mani $R$, Natural History of Nonstenotic, Asymptomatic Ulcerative Lesions of the Carotid Artery. Arch Surg 113, 1352, 1978 .

203 Moore WS, Bean BA,

Periorbital Doppler Examination (Ophthalmosonometry) and Supraorbical Photoplechysmography.

In: Practical Noninvasive Vascular Diagnosis.

eds. Kempczinski RF, Yao IST, Year Book Medical Publishers, Chicago, 1982.

204 Moore WS,

Pathogenetic Mechanisms of Cerebral Dystunction in Vascular Disease.

In: Vascular Surgery, second edition, ed. Rutherford RB, WB Saunders, Philadelphia, 1984.

205 Motomiya M, Karino T,

Flow Patterns in the Human Carovid Artery Bifurcation.

Stroke $15,50,1984$.

206 Mueller HR,

The Diagnosis of Internal Carotid Artery Occlusion by Directional Doppler Sonography of the Ophrhalmic Artery.

Neurology 22,816, 1972.

207 Mungas JE, Baker WH,

Amaurosis Fugax.

Stroke 8, 232, 1977 .

208 O'Donnell TF Jr, Pauker SG, Callow AD, Kelly J], McBride KJ, Korwin S,

The relative value of carotid noninvasive testing as determined by receser operator characteristic curves.

Surgery $87,9,1980$.

209 O'Donnell TF Jr, Erdoes L, Mackey WC, McCullough J, Shepard A, Heggerick P, IsnerJ, Callow $\mathrm{AD}$,

Correlation of B mode Uitrasound Imaging and Arteriography With Pathologic Findings at Carotid Endarterectomy.

Arch Surg 120, 443, 1985.

210 O'Hara PJ, Brewster DC, Darling RC, Hallert JW,

Oculopneumoplethysmography. Its Relationship to Intraperative Cerebrovasculas Hemodyrnamics.

Arch Surg 115, 1156, 1980.

211 Padayachee TS, Lewis $R$ R, Gosling RG,

Detection of carotid bifurcation disease: comparison of ultrasound tests with angiography.

B.] Surg 69, $218,1982$.

212 Paday achee TS, Lewis RR, Gosling RG,

Ultrasound Screening for Internal Carotid Disease-1. The Temporal Artery Occlusion

Test . Which Periorbital Artery.

Ultrasound in Med \& Biol 10, 13, 1984.

213 Padget $\mathrm{DH}_{\text {, }}$

The Circle of Willis. Irs Embryology and Anatomy.

In: Intracranial Arterial Aneurysms.

ed. Danon WE, Comstock Publishing Company INC., New York, 1947. 
214 Pantano P, Baron J-C, Lebrun-Grandie $\mathbb{P}$, Duquesnoy $\mathbb{N}$, Bousser M-G, Comar D, Regional Cerebral Blood Flow and Oxygen Consumption in Human Aging.

Stroke 15, 635, 1984.

215 Deronncau $P$, Hinglais $J$, Pellet $M$, Leger $F$;

Velocimetre sianguin par effet Doppler a emission ultra-sonore pulsee.

IOnde Electrique 50, 369, 1970.

216 Phillips DJ, Powers JE, Eyer MK, Blackshear WM Jr, Bodily KC, Strandness DEJr, BakerDW"

Detection of Peripheral Vascular Disease Using the Duplex Scanner III.

Ultrasound in Med \& Biol 6, 205, 1980.

217 Phillips DJ, Greene FM Jr, Langlois Y, Roederer GO, Strandness DE Jr,

Flow Velocity Patterns in the Carotid Bifurcations of Young, Presumed Normal Subjects.

Ultrasound in Med \& Biol 9, 39, 1983.

218 Pourcelot $L_{\text {s }}$

Continuous Wave Doppler Techniques in Cerebral Vascular Disturbances.

In: Doppler Ultrasound in the Diagnosis of Cerebrovascular Disease.

eds. Reneman RS, Hoeks APG, Research Studies Press, Chichester, 1982.

219 Ramirez-Lassepas $M$, Sandok BA, Burton $\mathbb{R C}$.

Chinical Indicators of Extracranial Carotid Artery Disease in Patients With Transient Symptoms.

Stroke 4, 537, 1973.

220 Raviola $C$, Baker JD, Cousins A, Defrte Dix,

Ewaluation of Extracranial Cerebrovascular Disease in the Hypertensive Patient with Ocular Pneumoplethysmography at $500 \mathrm{~mm} \mathrm{Hg}$.

An J Surg 144, 306, 1982.

221 Reid JM, Baker DW,

Physics and Ellectronics of the Ultrasonic Doppler Method.

In: Ultrasonographia Medica.

eds. Bock ], Ossoing K, Verlag der Wiener Medizinischen Akadenie, Wien, 1971.

222. Reid JM,

Sound and Uitrasound.

In: Cerebrovascular Evaluation with Doppler Ultrasound.

eds. Spencer MP, Reid JM, Martinus Nijhoff, The Hague, 1981.

223 Reilly LM, Ehrenfeld WK, Stoney RI,

Carorid digital subtraction angiography: The comparative roles of)intra-arterial and intravenous imaging.

Surgery $96,909,1984$.

224 Reneman RS, Spencer MP.

Difficulties in processing of an analogue Doppler flow signal; wirh special reference to 2 cro-crossing meters and quantication.

In: Cardiovascular Applications of Ultrasound.

ad. Reneman RS. North Holland Publishing Company, Amsterdam, 1974.

225 Reneman RS, Hocks APG.

Continuous-Wave and Pulsed Doppler Flowmeters - A General Introduction.

In: Bichocardiology.

ed. Bom N, Martinus Nijhotf, The Hague, 1977.

226 Reneman RS, Spencer MP,

Local Doppler Audio Spectra in Normal and Stanosed Carorid Arteries in Man.

Wh trasound in Med \& Biol 5, 1, 1979. 


\section{Reneman RS,}

What measurements are necessary for a comprehensive evaluation of the peripheral arterial circularion.

Cardiovascular Discases $8,435,1981$.

228 Reneman RS, Hocks APG,

Doppler Ultrasound - Principle, Advantages and Limitations.

In: Doppler Ultrasound in the Diagnosis of Cerebrowascular Disease.

eds. Reneman RS, Hoeks APG, Research Sudies Press, Chichester, 1982.

229 Reneman RS, Hoeks APG, Bruins Slot $\mathrm{H}$, Merode T van,

The On-line Recording of Velocity Profiles and Its Potential in the Diagnosis of Peripheral Arterial Lesions.

In: Noninvasive Assessment of the Cardiovascular System: Diagnostic Principles and Techniques.

ed. Diethrich EB, John. Wright, Boston, 1982.

230 Reneman RS, Pourcelot L, Hoeks APG,

Prospects and Conclusions.

In: Doppler Ulrasound in the Diagnosis of Cerebrovascular Disease.

eds. Reneman RS, Hocks APG, Research Studies Press, Chichester, 1982.

231 Reneman RS, Merode T van, Hick P, Hoeks APG,

Flow velocicy patterns in and distensibility of the carotid artery bulb in wolunters of various ages.

Circulation $71,500,1985$.

232 Reneman RS, Merode T van, Hick $\mathbb{P}$, Hoeks APG,

Cardiovascular Applications of Multi-gate Pulsed Doppler systems.

Cllorasound in Med \& Biol 12, 357, 1986.

233 Reneman RS, Merode T van, Hick P, Hoeks APG,

Velocity Patterns in and Vessel Wall Properties of the Carotid Artery Bifurcation in Man.

Applications of Doppler Ultrasound in Medicine.

in press, INSERM, Paris.

234 Riggs HE, Rupp C,

Variation in Form of Circle of Willis. The Relacion of the Variations to Collateral Circularion - Anatomic Analysis.

Arch Neurol 8, 8, 1963.

235 Rittgers SE, Thornhill BM, Barnes RW,

Quantitative Analysis of Carotid Artery Doppler Spectral Waveforms: Diagnostic Value of Parameters.

Ultrasound in Med \& Biol $9,255,1983$.

236 Roberts B, Hardesty WH, Holling HE, Reivich M, Toole JF,

Studies on extracranial cerebral blood flow.

Surgery $56,826,1964$.

237 Rogers L,

The Function of the Circulus Arteriosus of Willis.

Brain LXX, 171, 1947.

238 Ruff RL, Talman WT, Petito $F$ "

Transient Ischemic Attacks Associated with Hypotension in Hypertensive Patients witl

Carotid Artery Stenosis.

Stroke 12, 353, 1981 . 
239 Russell JB, Watson IM, Modi JR, Lambeth A, Sumner DS,

Digial subtaction angiography for evaluation of extracranial carotid occlusive disease:

Comparison with conwentional arteriography.

Surgery $94,604,1983$.

240 Rutherford RB, Hiat WR. Kreutzer EW,

The use of velocity ave form analysis in the diagnosis of carotid artery occlusive disease. Surgery $82,695,1977$.

241 Satiani $B$, Cooperman $M$, Clark M, Evans WE,

An Assessment of Caronid Phonoangrography and Oculoplethysmography in the Detection of Carotid Artery Stenosis.

Am J Surg 136,618, 1978 .

242 Satomura $S$,

Study of Flow Patterns in Peripheral Arteries by Ulcrasonics.

J Acoust Science Jpn 15, 151, 1959.

243 Scheinberg $P$, Meyer $\int S$, Reivich $M$, Sund TM, Waltz AG,

XIII. Cerebral Circulation and Metabolism in Srroke. Cerebral Circulation and Metabolism in Stroke Study Group.

Stroke 7, 213, 1976 .

244 Schouten HJA,

Measuring pairwise agrement among many observers.

Biom J 22, 497, 1980 .

245 Schwartz JA, Flanigan DP, Schuler JJ, Ryan TJ, Castronuowo JJ.

Indirect Assessment of Carotid Occlusive Disease by Ocular Pneumoplechysmography. $500 \mathrm{~mm} \mathrm{Hg}$ Vacuum Pressure Measurements and Ocular Pulse "Timing.

Stroke $15,521,1984$.

246 Seldinger $S$,

Catheter Replacement of the Needle in Percutaneous Arteriography.

Acta Radiol 39, 368, 1953.

247 Sheldon JJ, Janowitz W, Leborgne JM, Sivina M, Rojo N,

Intrawenous DSA of Extracranial Carotid Lesions: Comparison with Other Techniques and Specimens.

Am J Roentgenol 143,1289, 1984.

248 Smith D, Larsen JL,

On the Symmetry and Asymmetry of the Bifurcation of the Common Carotid Artery. $A$ Study of Bilateral Carotid Angiograms in 100 Adults.

Neuroradiology $17,245,1979$.

249 Smith RR, Russell WF, Percy ML,

Ultrastructure of Carotid Plaques.

Surg Newrol 14, 145, 1980.

250 Solberg LA, Eggen DA,

Jocalization and Sequence of Development of Atherosclerotic Lesions in the Carotid and Vertebral Arteries.

Circulation $43,711,1971$.

251 Spencer MP, Reid JM,

Quantitation of Carotid Stenosis with Concinuous-Wave (C-W) Doppler Ultrasound.

Stroke 10, 326, 1979. 


\section{Spencer MP,}

Full Capability Doppler Diagnosis.

In: Cerebrovascular Evaluation with Doppler Ultrasound.

eds. Spencer MP, Reid JM, Martinus Nijhoff, The Hague, 1981.

253 Spencer MP, Arts T,

Some Hemodynamical Aspects of Large Arteries.

In: Doppler Ultrasound in the Diagnosis of Cerebrowascular Disease.

eds. Reneman RS, Hoeks APG, Research Studies Press, Chichester, 1982.

254 Spencer MP, Zwiebel WI.

Frequency Spectrum Analysis in Doppler Diagnosis.

In: Introduction to Vascular Ultrasonography.

ed. Zwiebel WJ, Grune \& Stratton, New York, 1982.

255 Spetzler RF, Roski RA, Zabramski J,

Middle Cerebral Artery Perfusion Pressure in Cerebrovascular Occlusive Disease.

Scroke 14, 552, 1983.

256 Spitzler RL, Fleiss JL.

A Re-Analysis of the Reliability of Psychiatric Diagnosis.

Brit J Psychiat 125, 341, 1974.

257 Strandgaard S, Paulson $\mathrm{OB}$,

Cerebral Autoregulation.

Stroke $15,413,1984$.

\section{Strik F,}

De warde wan de oftalmodynamografic voor de vroege diagnostiek wan obstruerende processen an de carotiden.

NTvG 114, 1127, 1970.

259 Sumner DS, Russell $\rrbracket B$, Miles RD,

Are noninvasive tests sufficiently accurate to identify patients in need of carotid angiography?

Surgery $91,700,1982$.

260 Sumner DS,

Hemodynamics and Pathophysiology of Arterial Disease.

In: Vascular Surgery, second edition.

ed. Rutherford RB, WB Saunders, Philadelphia, 1984.

261 Suzuki I,

Comeal Pulsation and Corneal Pulse Waves.

Jpn J Ophthalmol 6, 190, 1962.

262 "Tada K, Nukada $T$, Yoneda S, Kuriyama Y, Abe H,

Assessment of the capacity of cerebral collateral circulation using ultrasonic Doppler technique.

INeurol Neurosurg Psychiat 38, 1068, 1975.

263 Thiele BL, Young JV, Chikos PM, Hirsch JH, Strandness DE Jr,

Correlation of arteriographic findings and symptoms in cerebrovascular disease.

Neurology $30,1041,1980$.

264 Thomas DJ, DuBoulay GH, Marshall 』, Pearson TC, Ross Russell RW, Symon L, Wetherley-Mein G, Zilkha E,

Effect of Haematocrit on Cerebral Blood Flow in Man.

Lancer $2,941,1977$. 
265 Thomas DH.

The Intluence of Blood Viscosity on Carebral Blood Flow and Symptoms.

In: Progress in Stroke Rescarch, 1 ,

eds. Greenhalgh RM, Rose FC, Piman Medical Publishing, Tunbridge Wells, 1979.

266 Toole JF, Janeway $R$, Choi $K$, Cordell $R$, Davis $C$, Johnston $F$, Miller $H S$,

"Transient Ischemic Artacks Due to Atherosclerosis. A Prospecrive Study of 160 Patients. Arch Neurol, 32, 5, 1975.

267 Towne JB, Bernhard VM, Sallesm Cunha S, Ray 1 , Supraorbital Doppler Exaluation in Vascuhr Surgery.

Medical Instrumentation $13,92,1979$.

268 Uematsu S, Yang A, Preziosi T], Kouba $\mathbb{R}$, Toung T]K,

Measurement of Carotid Blood Flow in Man and irs Clinical Applicanon.

Stroke 14,256,1983.

269 Ulrich WD,

Grundlagen und Merhodik der Ophahalmodynamometrie (ODM), Ophthalmodynamographie (ODG), Temporalisdynamographie (TDG).

Georg Thieme Verlag, Stuttgart, 1976.

270 Vagnini FJ, Kaplitt MJ, Frantz SL, Sawyer PN, Stein HL,

Reillame Carotid Artery $U \mid$ trasonography.

In: Noninvasive Assessment of the Cardiovascular System, Diagnostic Principles and Technigues.

ed. Diethrich EB, John Wright, Boston, 1982.

271 Weaver RG Jr, Howard G, Mckinney WM, Ball MR, Jones AM, Toole JF,

Comparison of Doppler Ultrasonography with Arteriograply of the Carotid Artery Bifurcation.

Stroke $1,1,402,1980$.

272 Weibel $J$, Ficids WS,

Atlas of Arteriography.

W.B. Saunders Comp, Philadelphia, 1969.

273 Weibal J, Ficlds WS,

Tortuosity, Coiling and Kinking of the Internal Carotid Artery. I : Etiology and Radiographic Anatomy.

Neurology $15,7,1965$.

274 Weintraub MI, Lambert D, Rothman AL,

Carotid Ultrasonography - The New "Gold Standard". Surgical and Angiographic Correlation.

Angiology 36, 19, 1985.

275 Weisman RE, Upson $\mid \mathrm{F}$,

Intra-Arterial pressure studies in patients with arterial insufficiency of lower extremities. Ann Surg 157, 501, 1963.

276 Wells PNT,

"The Possibility of Harmful Biological Effects in Ultrasonic Diagnosis.

In: Cardiovascular Applications of Ultrasound.

ed. Reneman RS, North Holland Publishing Company, Amsterdam, 1974.

277 Whisnant $\int \mathrm{P}$,

Multiple Particles Injected May All Go to the Same Cerebral Artery Branch. Letter to the Editor.

Stroke $13,720,1982$. 
278 Wiebers DO, Folger WN, Forbes GS, Younge BR, O'Fallon WM,

Ophthalmodynamometry and Ocular Pneumoplethysmography for Detection of Carotid Occlusive Disaese.

Arch Neurol 39,690, 1982.

279 Wise G, Parker J, Burckholder J.

Supraorbital Doppler studies, carorid bruits, and arteriography in unilateral ocular or cerebral ischemic disorders.

Neurology $29,34,1979$.

280 Yoneda S, Nukada T, Kimura $K$, Tanaka K, Ashida K, Asai T, Erani $H_{\text {, Imazumi M, }}$ Abe $\mathrm{H}$,

Evaluation of Cross-Circulation Through Circle of Willis Using An Ultrasonic Doppler Technique. Part I: Comparison between Blood Flow Velocity by Uhrasonic Doppler Flowmetry and Angiogram.

Stroke 12, 478, 1981 .

281 Yoneda S, Nukada T, Kimura K, Tanaka K, Ashida K, Asai T, Etani H, Imazumi M, Abe $\mathrm{H}$,

Evaluation of Cross-Circulation Through Circle of Willis Using an Ultrasonic Doppler "Technique. Part II: Comparison between Blood How Velociry by Doppler Flowmery and Cerebrovascular Resistance.

Siroke 12, 484, 1981.

282 Zagzebski JA, Madsen EL,

Physics and Instrumentation in Doppler and B-mode Ultrasonography.

In: Introduction to Vascular Ultrasonography.

ed. Zwiebel WJ, Grune \& Stratton, New York, 1982.

283 Zamir M, Brown $N$,

Pressure Rise at Arterial Bifurcations.

Angiology 35, 645, 1984 .

284 Zarins CK, Giddens DP, Glagov $S$,

Atherosclerotic Plaque Distribution and Flow Velocity Profiles in the Carotid Bifurcation.

In: Cerebrovascular Insufficiency.

eds. Bergan JJ, Yao JST, Grune \& Stratton, New York, 1983.

285 Zbornikova $\mathrm{V}$, Akesson $\rrbracket-A$, Lassuik $C_{\text {. }}$.

Diagnosis of carotid artery disease - Comparison berween Directional Doppler, Duplex

Scanner and Angiography.

Acra Neurol Scand 65, 335, 1982.

286 Zwiebel WJ, Crummy AB,

Sources of Error in Doppler Diagnosis of Carotid Occlusive Disease.

Am 』Roentgenol 137, 231, 1981 .

287 Zwiebel WJ,

Doppler Carotid Imaging and Interpretation of Abnormal Carotid Flow Signals.

In: Introduction to Vascular Ultrasonography.

ed. Zwiebel WJ, Grunc \& Stratton, New York, 1982.

288 Zwiebel W],

High-resolution B-mode and Duplex Carotid Sonography.

In: Introduction to Vascular Ultrasonography

ed. Zwiebel WJ, Grune \& Stratton, New Yorth, 1982. 
289 Zwiebel WJ Zagzebski J , Crummy AB, Hirscher M, Correlation of Peak Doppler Frequency with Lumen Narrowing in Carotid Stenosis. Suroke $13,386,1982$.

290 Zwiebel WJ Strother CM, Austin CW, Sacket JF, Comparison of Ulrasound And IV-DSA For Carotid Evallation.

Stroke 16,633, 1985. 


\section{CURRICULUM VITAE}

17-6-1949 : geboren te Hooge en Lage Zwaluwe

$1961-1965$ : Mulo-B opleiding, Meyenhage ULO, Rotterdam

$1965-1968$ : HBS-B opleiding, Joh. Calvijn Lyceum, Rotterdam

$1968-1974$ : studie Geneeskunde, Rijksuniversiteit Utrecht

29-11-1974 : artsexamen

1975 : dienstplichtig militair arts

1-1-1976-

1-1-1982 : opleiding chirurgie, Catharina Ziekenhuis te Eindhoven, op-

$1-1-1982-$ leiders: dr. Q.A.M. Eijsbouts, dr. J.G. Prins

1-9-1983 : chirurg, chef de clinique, Catharina Ziekenhuis te Eindhoven

sinds 15-9-1983: chirurg in het ziekenhuis "De Goddelijke Voorzienigheid" te Sittard. 